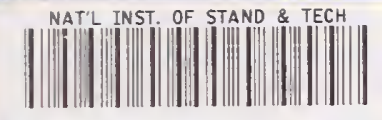

All106 l38607

u.o. verafinumery of bunisienive

National Bureau of Standards

NBS Special Publication 700-1 Industrial Measurement Series

\title{
A Primer for Mass Metrology
}

NBS NBS NBS NBS NBS NBS NBS NBS NBS NBS IS NBS NBS NBS NBS NBS NBS NBS NBS NBS NBS NBS NBS NBS NBS NBS NBS NBS NBS NBS NBS IS NBS NBS NBS NBS NBS NBS NBS NBS NBS NBS NBS NBS NBS NBS NBS NBS NBS NBS NBS NBS N IS NBS NBS NBS NBS NBS NBS NBS NBS NBS NBS NBS NBS NBS NBS NBS NBS NBS NBS NBS NBS N iS NBS NBS NBS NBS NBS NBS NBS NBS NBS NBS NBS NBS NBS NBS NBS NBS NBS NBS NBS NBS iS NBS NBS NBS NBS NBS NBS NBS NBS NBS NBS NBS NBS NBS National Bureau of Standards NBS NBS N iS NBS NBS NBS NBS NBS NBS NBS NBS NBS NBS NBS NBS NBS NBS NBS NBS NBS NBS NBS NBS N BS NBS NBS NBS NBS NBS NBS NBS NBS NBS NBS NBS NBS NBS NBS NBS NBS NBS NBS $N$ BS NBS NBS NBS NBS NBS NBS NBS NBS NB⿺ 
he National Bureau of Standards ${ }^{1}$ was established by an act of Congress on March 3, 1901. The Bureau's overall goal is to strengthen and advance the nation's science and technology and facilitate their effective application for public benefit. To this end, the Bureau conducts research and provides: (1) a basis for the nation's physical measurement system, (2) scientific and technological services for industry and government, (3) a technical basis for equity in trade, and (4) technical services to promote public safety. The Bureau's technical work is performed by the National Measurement Laboratory, the National Engineering Laboratory, the Institute for Computer Sciences and Technology, and the Center for Materials Science.

\section{The National Measurement Laboratory}

Provides the national system of physical and chemical measurement; coordinates the system with measurement systems of other nations and furnishes essential services leading to accurate and uniform physical and chemical measurement throughout the Nation's scientific community, industry, and commerce; provides advisory and research services to other Government agencies; conducts physical and chemical research; develops, produces, and distributes Standard Reference Materials; and provides calibration services. The Laboratory consists of the following centers:
- Basic Standards ${ }^{2}$

- Radiation Research

- Chemical Physics

- Analytical Chemistry

\section{The National Engineering Laboratory'}

Provides technology and technical services to the public and private sectors to address national needs and to solve national problems; conducts research in engineering and applied science in support of these efforts; builds and maintains competence in the necessary disciplines required to carry out this research and technical service; develops engineering data and measurement capabilities; provides engineering measurement traceability services; develops test methods and proposes engineering standards and code changes; develops and proposes new engineering practices; and develops and improves mechanisms to transfer results of its research to the ultimate user. The Laboratory consists of the following centers:
- Applied Mathematics

- Electronics and Electrical Engineering ${ }^{2}$

- Manufacturing Engineering

- Building Technology

- Fire Research

- Chemical Engineering ${ }^{2}$

\section{The Institute for Computer Sciences and Technology}

Conducts research and provides scientific and technical services to aid Federal agencies in the selection, acquisition, application, and use of computer technology to improve effectiveness and economy in Government operations in accordance with Public Law 89-306 (40 U.S.C. 759), relevant Executive Orders, and other directives; carries out this mission by managing the Federal Information Processing Standards Program, developing Federal ADP standards guidelines, and managing Federal participation in ADP voluntary standardization activities; provides scientific and technological advisory services and assistance to Federal agencies; and provides the technical foundation for computer-related policies of the Federal Government. The Institute consists of the following centers:
- Programming Science and Technology

- Computer Systems Engineering

\section{The Center for Materials Science}

Conducts research and provides measurements, data, standards, reference materials, quantitative understanding and other technical information fundamental to the processing, structure, properties and performance of materials; addresses the scientific basis for new advanced materials technologies; plans research around cross-country scientific themes such as nondestructive evaluation and phase diagram development; oversees Bureau-wide technical programs in nuclear reactor radiation research and nondestructive evaluation; and broadly disseminates generic technical information resulting from its programs. The Center consists of the following Divisions:
- Inorganic Materials

- Fracture and Deformation ${ }^{3}$

- Polymers

- Metallurgy

- Reactor Radiation 


\section{NBS Special Publication 700-1 Industrial Measurement Series}

A Primer for
Mass Metrology

K.B. Jaeger

Measurement Standards Laboratory

Lockheed Missiles and Space Company, Inc.

Sunnyvale, CA 94086

and

R.S. Davis

Center for Basic Standards

National Measurement Laboratory

National Bureau of Standards

Gaithersburg, MD 20899

November 1984

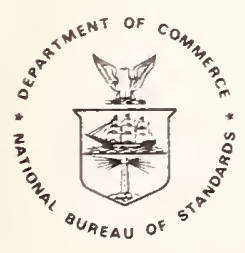

U.S. Department of Commerce Malcolm Baldrige, Secretary

National Bureau of Standards

Emest Ambler, Director 
Library of Congress

Catalog Card Number: 84-601090

National Bureau of Standards

Special Publication 700-1

Natl. Bur. Stand. (U.S.),

Spec. Publ. 700-1,

85 pages (Nov 1984)

CODEN: XNBSAV
U.S.Government Printing Office

Washington: 1984

For sale by the Superintendent of Documents,

U.S. Government Printing Office, Washington, DC 20402 
FOREWORD

When the National Bureau of Standards was established more than 80 years ago, it was given the specific mission of aiding manufacturing and commerce. Today, NBS remains the only Federal laboratory with this explicit goal of serving U.S. industry and science. Our mission takes on special significance now as the country is responding to serious challenges to its industry and manufacturing--challenges which call for government to pool its scientific and technical resources with industry and universities.

The links between NBS staff members and our industrial colleagues have always been strong. Publication of this new Industrial Measurement Series, aimed at those responsible for measurement in industry, represents a strengthening of these ties.

The concept for the series stems from the joint efforts of the National Conference of Standards Laboratories and NBS. Each volume will be prepared jointly by an industrial specialist and a member of the NBS staff. Each volume will be written within a framework of industrial relevance and need.

This publication, A Primer for Mass Metrology, represents the first of what we anticipate will be a long series of collaborative ventures that will aid both industry and NBS.

6. Ambles.

Ernest Ambler, Director

ii i 
Klaus Jaeger

Dr. Klaus Jaeger was educated at Syracuse University (B.S., Physics 1965; Ph.D., High Energy Physics, 1970). He was employed at Argonne National Laboratory from 1970 to 1980 and Brookhaven National Laboratory from 1980 through 1981. During these periods $\mathrm{Dr}$. Jaeger was engaged in laboratory research in neutrino and meson interaction physics, bubble chamber experiments, particle beam designs and superconducting magnet technology. In addition to high energy physics his experience includes electronics, computer science and cryogenics.

In January 1982 Dr. Jaeger joined Lockheed Missiles and Space Company, Sunnyvale, California as a research specialist responsible for upgrading all primary measurement standards required by the organization. Since October 1983 he has been the supervisor responsible for primary electrical measurement standards. Dr. Jaeger resides in Saratoga, California.

\section{Richard S. Davis}

Dr. Richard Davis was educated at Brown University (B.S., Physics, 1967) and the University of Maryland (Ph.D., Solid State Physics, 1972). His fields of study include experimental solid state physics, theoretical and experimental problems in fluid dynamics, properties of materials at very low temperatures, superfluid properties of liquid helium, electronics, absolute electrical measurements and basic mass metrology.

Dr. Davis joined the National Bureau of Standards in 1972. His laboratory research programs at NBS have been concerned with the improvement of the measured value of the Faraday (the fundamental constant of electrochemistry), high voltage capacitor calibrations, the accurate determination of air density effects in mass measurements and improved methods for mass comparison at the highest levels of accuracy. Dr. Davis resides in Washington, D.C. 

Foreword

About the Authors ........................ . . . v

1. Introduction . . . . . . . . . . . . . . . . . . . . 1

2. Basic Mass Equations ...................... . . . . . 2

2.1. True Mass and Apparent Mass ................ 8

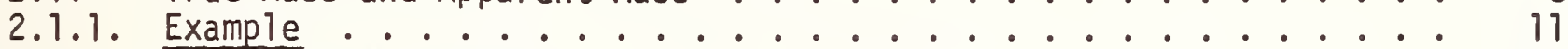

2.1.2. Reference Materials .................. . . 15

2.1.3. Reporting Apparent Mass . . . . . . . . . . . . . . . . 17

2.1.4. Comparing Two Apparent Masses . . . . . . . . . . . . . . 17

2.1.5. Sources of Error in Buoyancy Corrections . . . . . . . . . . . 19

2.2. Air Density . . . . . . . . . . . . . . . . . . . 21

2.3. Sensitivity Arguments . . . . . . . . . . . . . . . 22

2.3.1. Humidity . . . . . . . . . . . . . . . . . . 23

2.3.2 Temperature ...................... . . . . 24

2.3.3 Pressure .......................... . . . . . . . . 24

2.3.4. Overall Uncertainty of Air Density. . . . . . . . . . . . . . 24

2.3.5. Effects of Small Changes in Air Density on the Mass Value . . . 25

2.4. Measuring Equipment for Air Density .............. 26

2.4.1. Humidity..................... . . . . . 26

2.4.2. Temperature ...................... 26

2.4.3. Barometric Pressure ................ . . 26

3. Weighing Methods .. . . . . . . . . . . . . . . . . 28

3.1. Direct Weighing ...................... 29

3.2. Direct-Reading Weighing . . . . . . . . . . . . . 30

3.3. Substitution Weighing ................. . 30

3.3.1. Single Pan Balance................... . 30

3.3.1.1. Single Substitution .. . . . . . . . . . . . . . 30

3.3.1.2. Double-Substitution . . . . . . . . . . . . . . . . 35

3.3.2. Two-Pan Equal-Arm Balance . . . . . . . . . . . . 36

3.3.2.1. Single Substitution ................. . . . . 36

3.3.2.2. Double-Substitution ................ . . . . 36

3.4. Transposition Weighing . . . . . . . . . . . . . . 37

3.4.1. Single Transposition . . . . . . . . . . . . . . . 37

3.4.2. Double Transposition . . . . . . . . . . . . . . . . . 38

3.5. Weighing on Electronic Balances . . . . . . . . . . . . . 39

4. Program Types . . . . . . . . . . . . . . . . . . . . . . 4 41

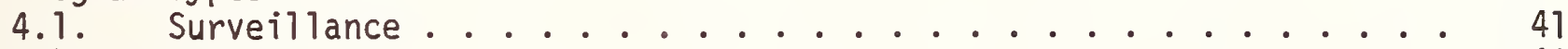

4.1.1 Type I......................... . . . . . . 4 42

4.1.2. Type II . . . . . . . . . . . . . . . . . 4 43

4.1.3. Surveillance Limits .. . . . . . . . . . . . . . . . . . . 49

4.1.4 Identifying Weights Which Have Changed . . . . . . . . . . . . . 52

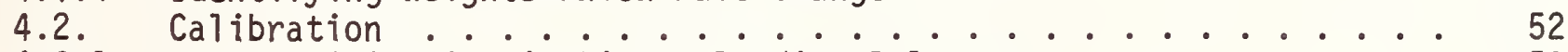

4.2.1. Trend Elimination in Direct Reading Balances . . . . . . . . . . 53

4.2.2. Designs . . . . . . . . . . . . . . . . . . . . 54

4.2.3. Statistical Checks . . . . . . . . . . . . . . . 57

5. Conclusion . . . . . . . . . . . . . . . . . . . 62

6. References . . . . . . . . . . . . . . . . . . 64 
Appendix A . . . . . . . . . . . . . . . . . . . . . . . . Al

Appendix B . . . . . . . . . . . . . . . . . . . . . . . . . . .

Appendix C . . . . . . . . . . . . . . . . . . . . . . . . . . . . . . . . . .

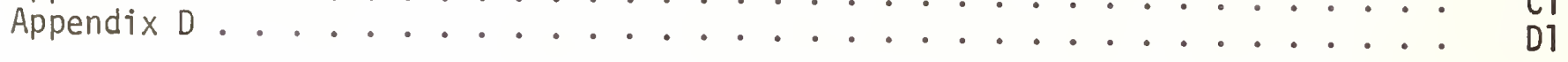




\author{
K. B. Jaeger \\ Measurement Standards Laboratory \\ Lockheed Missiles and Space Company, Inc. \\ Sunnyvale, CA 94086 \\ and \\ R. S. Davis \\ Center for Basic Standards \\ National Bureau of Standards \\ Gaithersburg, MD 20899
}

\title{
1. INTRODUCTION
}

Much of the mass program at the National Bureau of Standards has been documented during the last two decades [1-8]. What we attempt here is to explain the relevance of these publications to mass metrology conducted at laboratories whose standards are calibrated by NBS. We try to begin with basic physics concepts deriving the essential results with a minimum of rigor. The reader should always consult the appropriate reference for a more sophisticated treatment.

We have also tried to use a consistent notation. Consequently, our notation may vary slightly from that found in the references cited above.

Many examples are provided in the belief that understanding of a general case is much easier if a special case is understood well. Cautionary statements are provided at places in the text where important practical complications may exist.

In general, we have strived to be understandable rather than scholarly where the combination was elusive.

CAUTION: This document is incomplete. Many extremely important topics--manufacture, specification, cleaning, handling, and storage of weights being among them--have been omitted. The first two of these subjects are treated in ref. [14].

Our ultimate goal is to outline the way in which mass measurements can be made to acceptable metrological accuracies. There are three major concepts which must be explored but which are somewhat interrelated - what one means by "mass;" how one can use a measuring device to determine the mass of an unknown object in terms of an accepted unit, for example, the kilogram; and how to assign a realistic uncertainty to the results. It will be useful to introduce some basic equations of physics in order to make our arguments precise. The first major step is to explore the basic properties of a mass-measuring device, or balance, and then see how the device can be put to the service of metrology. Mathematically, this means that the first step is to derive the basic equations of weighing. The remainder of our efforts modify the basic equations in order to make them practical. We also show their relevance to mass metrology. 
In order to derive the basic equations of weighing, we need a precise notion of mass. For the purpose of this discussion we will introduce mass through the familiar equation:

$$
\begin{gathered}
\vec{F}=M \vec{a} \\
\text { (Force }=\text { Mass } \times \text { Acceleration) }
\end{gathered}
$$

That is, each object possesses a property called "mass" which appears in eq. (1) as the constant of proportionality between a force applied to that object and the resulting acceleration of the object. Note that both force and acceleration are vector quantities: they have a direction associated with them. One should also note that mass is always a positive number (that is, for example, the acceleration is always in the same direction as the gravitational force, never opposite to it). These comments about mass are consistent with the qualitative idea of the mass of an object being a measure of the "amount of substance" in the object.

Unfortunately, our intuitive notions of "mass" are often confused with "weight." Such confusion is unacceptable for science and metrology. Therefore we will now see how the notion of weight differs both logically and practically from that of mass.

We can take it as an experimental fact that over a small plane area of the earth's surface (șuch as the space occupied by a metrology laboratory) the acceleration of gravity, $\vec{g}$, is essentially constant. Since $\vec{g}$ is a vector quantity, constant $\vec{g}$ implies that, over a small area of the earth's surface, the direction of $\vec{g}$ is also practically constant. That is, the acceleration vectors are all parallel and define the direction "down." In this approximation, we can replace g by a numerical constant, $\bar{g}$.

The gravitational force on an object of mass $M$ is then:

$$
F_{1}=M \bar{g} \text {. }
$$

The weight of an object of mass $M$ is defined as $F_{\gamma^{\prime}}$. That is, weight is a force, not a mass.

$$
\text { Using } W=F_{1} \text {, we have }
$$

$$
W=M \bar{g}
$$

From what was said about $\bar{g}$, it is clear that weight is not a constant property of matter, but depends on location. Consider, for example, a body of mass $M$ taken out into deep space such that the gravitational forces are negligible (they are never zero). In this case, we can approximate (3) as

$$
W^{\prime}=M \bar{g} \quad 0
$$

which holds since

$$
\bar{g} \quad 0 ;(M \neq 0) \text {. }
$$

This definition was adopted for international use by the General Conference for Weights and Measures (C.G.P.M.) in 1901. 
Hence, the body still has mass $M$ but its weight is zero. It is only because most people live exclusively on the surface of the earth that weight and mass are often confused.

Let us now consider the action of a scale or balance - the device which we will use to measure mass. There are a great many schemes in use for constructing scales and balances. All have their strengths and weaknesses but most are designed to approximate the workings of the following hypothetical device:

This device is a "black box" which has a pan on which to place an object and an indicator which reads zero when the pan is empty and some other number when an object is placed on the pan (fig. 1). Our ideal scale has the following two desirable properties:

1) The indicator always reads the same amount to any desired precision when the same object is put on the pan at the same conditions of temperature, barometric pressure, relative humidity, etc.

2) If any two objects are put on the pan, the indicator reading is the sum of the readings for each object placed individually.

The first property means that the imprecision of the balance readings is zero. The second property means that the balance is perfectly linear. Later we will show how to use real balances which cnly approximate these two important features.

A final property of our idealized balance, which it shares with actual scales and balances is that the indicator responds to a force on the pan, not a mass. In other words, our balance would give different readings on the moon than on earth (though properties 1) and 2) would be unaffected).

When the balance has reached an equilibrium condition, the sum of all forces acting on the system must be zero. We then have

$$
\sum_{i=1}^{n} \vec{F}_{i}=0
$$

and since the forces acting on the system are known to act in one direction and its exact opposite (i.e. up and down) we can once again ignore the vector notation and write

$$
\sum_{i=1}^{n} F_{i}=0
$$

where $n=$ total number of forces acting on the system.

The forces acting on the object placed on the pan of the balance are:

$F_{1}=M \bar{g} \quad$ gravitational force

$F_{2}$, balance force exerted by the balance via deflection of a beam, stretching of a spring, or some other method. Obviously this force has to be opposite to $F_{1}$.
Since $F_{2}$ acts opposite to $F_{1}$, we adopt the convention

$$
F_{2}=-k \cdot \theta_{1} \cdot \bar{g}
$$




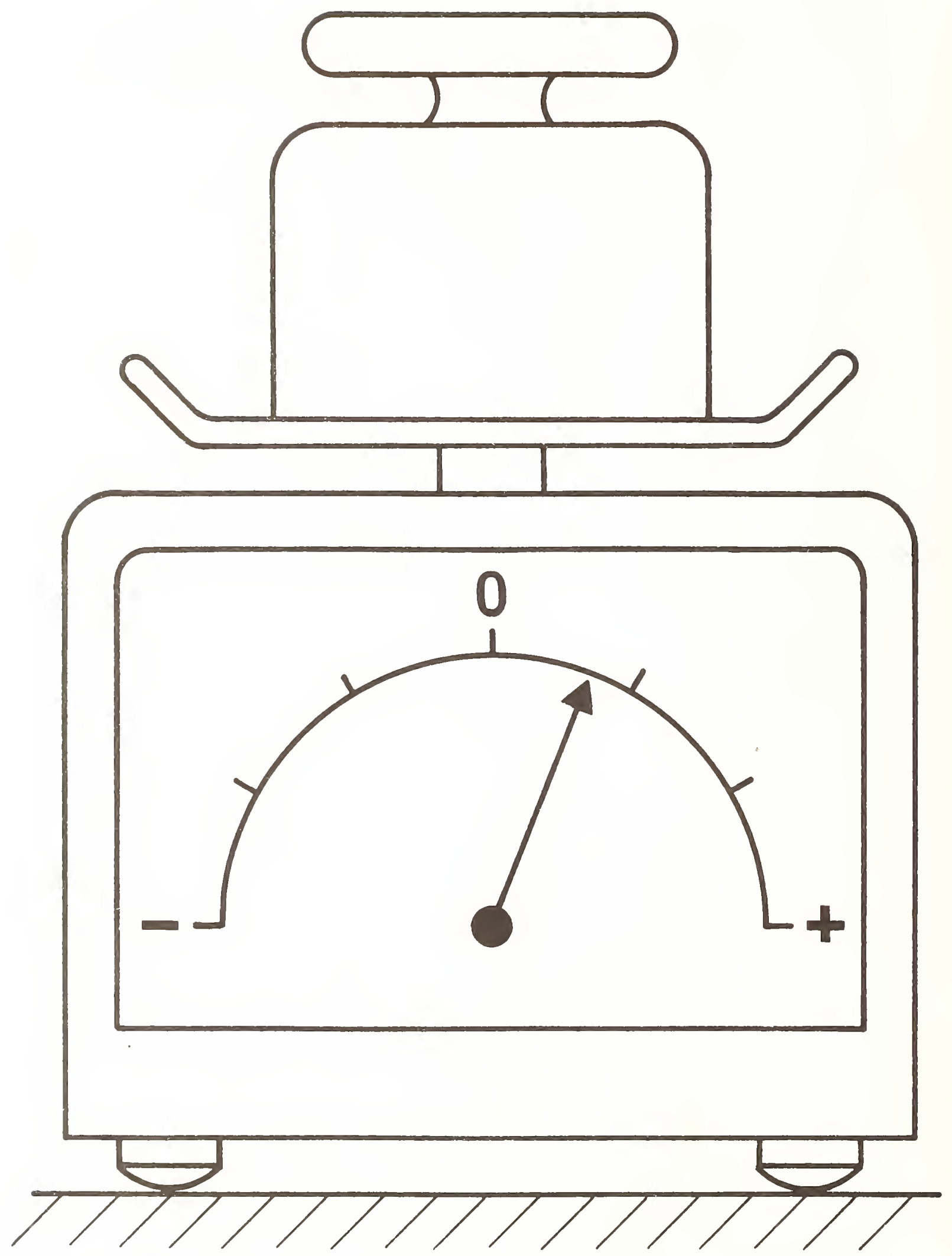

Figure 1. An idealized scale. The indicating dial is marked in equally spaced increments. The number pointed to by the needle can be resolved to any desired precision. 
where $\theta$ is the balance reading and $k$ is a positive constant (yet to be evaluated) which converts balance readings to mass units.

In addition to these main forces, we have to consider buoyant, electrostatic, and electromagnetic forces on the object. Of these three only the first one plays a significant role in usual metrology and we now look at the effect.

Under the influence of gravity, the force on a body submerged in a fluid is equal in magnitude but opposite in direction to the weight of the displaced medium. This upward force is known as the "buoyant force." From the definition we see that

$$
\mathrm{F}_{3}=-\mathrm{m} \overline{\mathrm{g}}
$$

where $m=$ mass of displaced medium. The buoyant force was first quantified by Archimedes in the third century B.C. We also can write

so that

$$
m=V \rho_{m}
$$

where

$$
F_{3}=-V_{\rho_{m}} \bar{g}
$$

$$
\begin{aligned}
V & =\text { volume of the displaced medium } \\
& =\text { volume of object } \\
\rho_{m} & =\text { density of medium } \equiv m / V .
\end{aligned}
$$

With these three forces, we can now write eq. (4) as

or

$$
F_{1}+F_{2}+F_{3}=0
$$

$$
F_{1}+F_{3}=-F_{2} \text {. }
$$

Substituting from eqs. (2), (5) and (6) yields

$$
\begin{aligned}
& M \bar{g}-\rho_{m} V \cdot \bar{g}=k \theta_{1} \bar{g} \\
& \left(M-\rho_{m} V\right) \bar{g}=k \theta_{l} \bar{g} \\
& \left(M-\rho_{m} V\right)=k \theta_{1} .
\end{aligned}
$$

It is very important to realize from eq. (7a) that if the weighing is done in a vacuum chamber then the $F_{3}$ term drops out and eq. (7a) reduces to

$$
M \bar{g}=k \theta_{j} \bar{g} \quad \text {. }
$$

Furthermore, the medium or environment can be any fluid--air, water, oil, etc. For most applications and for practical purposes, the medium is usually air. Even though air is a mixture of gases and the density is quite small compared to densities of objects usually measured, the correction term, $F_{3}$, cannot be ignored by metrologists, as we shall see.

Let us now consider the weighing of two objects of mass $M_{1}$ and $M_{2}$ under the same conditions, i.e. in the same medium. We then have from eq. (7a)

$$
\begin{aligned}
& \bar{g}\left(M_{1}-\rho_{m} V_{1}\right)=k \theta_{1} \bar{g} \\
& \bar{g}\left(M_{2}-\rho_{m} V_{2}\right)=k \theta_{2} \bar{g} .
\end{aligned}
$$


The density of an object is its mass devided by its volume. That is,

$$
\rho_{1} \equiv M_{1} / V_{1}
$$

where $\rho_{7}$ is the density of object 1 . We can, therefore, always replace a quantity such as

$$
M_{1}-\rho_{m} V_{1}
$$

by the identical quantity

$$
M_{1}\left(1-\rho_{m} / \rho_{1}\right) \text {. }
$$

The choice of representation is completely optional, although the proper choice can often simplify a calculation. For the present, we will continue with the volume representation.

Subtracting eq. (7e) from 7 (d) yields

$$
\bar{g}\left(M_{1}-\rho_{m} V_{1}\right)-\bar{g}\left(M_{2}-\rho_{m} V_{2}\right)=\bar{g} k \theta_{1}-\bar{g} k \theta_{2}
$$

and by setting

$$
\theta_{1}-\theta_{2}=\Delta \theta
$$

we have

$$
\bar{g}\left(M_{1}-\rho_{m} V_{1}\right)-\bar{g}\left(M_{2}-\rho_{m} V_{2}\right)=\bar{g} k \Delta \theta .
$$

Cancelling $\bar{g}$ yields

$$
M_{1}-M_{2}=k \Delta \theta+\rho_{m}\left(V_{1}-V_{2}\right) .
$$

Equation (9) is one of the fundamental equations in mass metrology and is known as the "Weighing Equation." Its importance will become evident in Section 3. For now we simply point out that, using the best balances available, one can determine $\Delta \theta$ to much higher accuracy than either $\theta_{1}$ or $\theta_{2}$. Thus eq. (9) turns out to be more usefut than eq. (7b).

In most cases, the laboratory conditions are such that the medium is air, in which case

$$
\rho_{A}=\rho_{m}=\text { density of air }
$$

and eq. (9) becomes

$$
M_{1}-M_{2}=k \Delta \theta+\rho_{A}\left(V_{1}-V_{2}\right)
$$

Note: When we refer to "weighing" in this text, we will always mean the process which determines the product $k \cdot \theta$. It should be clear from the above paragraphs that finding the mass of an object involves more than just weighing the object. Also, we have no future need to refer to "weight" as defined by (3). From this point on, the term "weight" will be reserved solely to designate an object manufactured to have particular nominal mass. For example, a "l-g weight" is an object whose mass is close to $1 \mathrm{~g}$. 
From eqs. (9) and (10) it is obvious the condition

$$
M_{1}-M_{2}=k \Delta \theta
$$

can exist if and only if

$$
\rho_{m}\left(V_{1}-V_{2}\right) \equiv 0
$$

This can only hold if

or

$$
\text { 1) } \rho_{m} \equiv 0 \quad \text { i.e., the measurement is done in vacuum }
$$

2) $v_{1} \equiv v_{2}$.

The first case is usually not encountered in metrology. The second case can be approximated quite easily. Consider for example two weights of nearly equal mass, such that

$$
M_{1} \approx M_{2},
$$

made from the same billet of alloy. In this case, the densities are equal so that

$$
\rho_{1}=\rho_{2} \equiv \rho \text {. }
$$

Since

$$
v_{1}=\frac{M_{1}}{\rho_{1}} \text { and } v_{2}=\frac{M_{2}}{\rho_{2}}
$$

we have from (10)

$$
\begin{aligned}
& M_{1}-M_{2}=k \Delta \theta+\rho_{A}\left(\frac{M_{1}}{\rho_{1}}-\frac{M_{2}}{\rho_{2}}\right) \\
& M_{1}-M_{2}=k \Delta \theta+\rho_{A}\left(\frac{M_{1}-M_{2}}{\rho}\right) \\
& \left(M_{1}-M_{2}\right)\left(1-\frac{\rho_{A}}{\rho}\right)=k \Delta \theta \\
& M_{1}-M_{2}=\frac{k \Delta \theta}{1-\rho_{A} / \rho} \\
& M_{1}-M_{2} \simeq k \Delta \theta
\end{aligned}
$$

because $\rho_{A} \ll \rho$ and $M_{1}-M_{2}$ is already known to be a small number.

Relations such as that expressed by eq. (1) or (10) are of use to metrology only if all variables are expressed in a consistent set of units. In what follows, we will assume that we are working in the International System of Units (also known as the Systeme International des Unités, or simply, the S.I.). 
The unit of mass in the S.I. is the kilogram. By definition, one kilogram is exactly equal to the mass of an object known as the International Prototype Kilogram, or I.P.K. This object is made of a platinum-iridium alloy and is stored at the International Bureau of Weights and Measures in Sèvres, France. All laboratory mass standards must ultimately be traceable to the I.P.K.

\subsection{True Mass and Apparent Mass}

So far, the "mass" we have considered is that found in Newton's basic equation (i.e., eq. (1)). For that reason, this concept of mass is sometimes called "true mass". It is a quantity intrinsic to the object alone and not to its situation. We have also seen that, in a vacuum, the force registered by the balance is exactly proportional to the Newtonian mass. For this reason the "true" mass is also known as the "vacuum" mass. "The terms "mass", "true mass" and "vacuum mass" are interchangeable.

The need to apply buoyancy corrections to mass measurements has led to the adoption of so-called "apparent" masses. As explained below and in Appendix A, use of apparent mass can simplify the job of both the weight manufacturer and the metrologist. The drawback, however, is that one must learn a new concept (that is, apparent mass).

of limited scientific significance, the apparent mass approach nevertheless often simplified calculations for the metrologist at a time when exact computations were tedious (either done by hand or through nomographs) and difficult to check. Today, when every metrologist has access, at the very least, to a digital pocketcalculator, the argument for apparent masses is less compelling. Nevertheless, both for completeness and because commercial weights are still manufactured on the basis of apparent mass, we cannot avoid this topic.

The fact is that most mass metrology is carried out: (1) in air; (2) in laboratories near sea level; and (3) at a temperature of $\sim 20^{\circ} \mathrm{C}$.

Air at such conditions has a density nearly equal to $1.2 \mathrm{mg} / \mathrm{cm}^{3}$. Fluctuations about the mean density are known rarely to exceed 3 percent. Thus 97 percent of the buoyancy correction can be "built into" the calibration of a weight so that small deviations from the correction are all that need be considered.

Let us carry through an example by defining a reference metal of density $\rho_{R}$.

Let

$$
\begin{aligned}
M_{R}^{T}= & \text { the true mass of an object made of reference material } R . \\
\text { (This object need not actually exist.) } & \\
\rho_{R}= & \text { the density of the reference material } \\
\rho_{0}= & 1.2 \mathrm{mg} / \mathrm{cm}^{3}=\text { the reference density of air. (This is very } \\
& \text { nearly the density of air at } 20{ }^{\circ} \mathrm{C}, 50 \% \text { humidity, and } 760 \\
& \mathrm{~mm} \mathrm{Hg} \text { pressure.) } \\
t_{0}= & 20{ }^{\circ} \mathrm{C}=\text { reference temperature. }
\end{aligned}
$$

If one actually were to weigh an object under vacuum conditions to determine its mass, one would have to be certain that the object weighed was sufficiently stable-that is, no gasses normally adsorbed on the surface at atmospheric pressure are vacuumed off, the weight itself does not "out-gas," etc. The equality between vacuum mass and true mass assumes that the object being weighed is stable. 
We now define the "apparent" mass of an object as follows: The "apparent" mass $M_{X}^{A}$ of an object $X$ is equal to the "true" mass $M_{R}^{\top}$ of just enough reference material to produce a balance reading equal to that produced by $X$ if the measurements are done at temperature $t_{0}$ in air of density $\rho_{0}$ (see fig. 2 ).

Stated another way, we first define specific, unique weighing conditions:

i. the air density is $1.2 \mathrm{mg} / \mathrm{cm}^{3}$

ii. the temperature is $20^{\circ} \mathrm{C}$ (it is necessary to specify temperature because the volume of a weight depends slightly on temperature.)

Next we imagine a mass comparison between an object $X$ and an assembly of known objects $R$. The definition specifies that the $R$ objects shall all have a density equal to $\rho_{R}$. The mass of the $R$ objects is then adjusted until

$$
\Delta \theta=\theta_{R}-\theta_{X}=0 \text {. }
$$

When these conditions are all satisfied, $M_{R}^{\top}$ is by definition equal to the apparent mass of $X$. We never really have to carry out the experiment because from eq. (9) we have

$$
M_{X}^{\top}-M_{R}^{\top}=k \Delta \theta+\rho_{0}\left(V_{X}-V_{R}\right)
$$

where $V_{R}$ is given by $V_{R}=M_{R}^{\top} / \rho_{R}$. We can rewrite this relation using density notation:

so that

$$
M_{X}^{\top}-\rho_{0} V_{X}-M_{R}^{\top}+\rho_{0} V_{R}=k \Delta \theta
$$

$$
M_{X}^{\top}\left(1-\rho_{0} / \rho_{X}\right)-M_{R}^{\top}\left(1-\rho_{0} / \rho_{R}\right)=k \Delta \theta
$$

and with

$$
\begin{gathered}
\Delta \theta=0 \\
M_{R}^{\top}=\frac{M_{X}^{\top}\left(1-\rho_{0} / \rho_{X}\right)}{\left(1-\rho_{0} / \rho_{R}\right)} \equiv M_{X}^{A} .
\end{gathered}
$$

Note that the denominator is simply a constant whose value is the same for any weight, $X$. Also, for $\rho_{X}$ greater than $1 \mathrm{~g} / \mathrm{cm}^{3}$ (the density of water), $M_{X}^{A}$ will be within 0.2 percent of $M_{X}^{\top}$. Furthermore, eq. (11) states that the apparent mass can be obtained by multiplying the true mass by a buoyancy factor.

The usefulness of apparent mass and hence eq. (11), as well as the subtle distinctions between true and apparent mass are shown in the next five subsections. We begin with an example. 


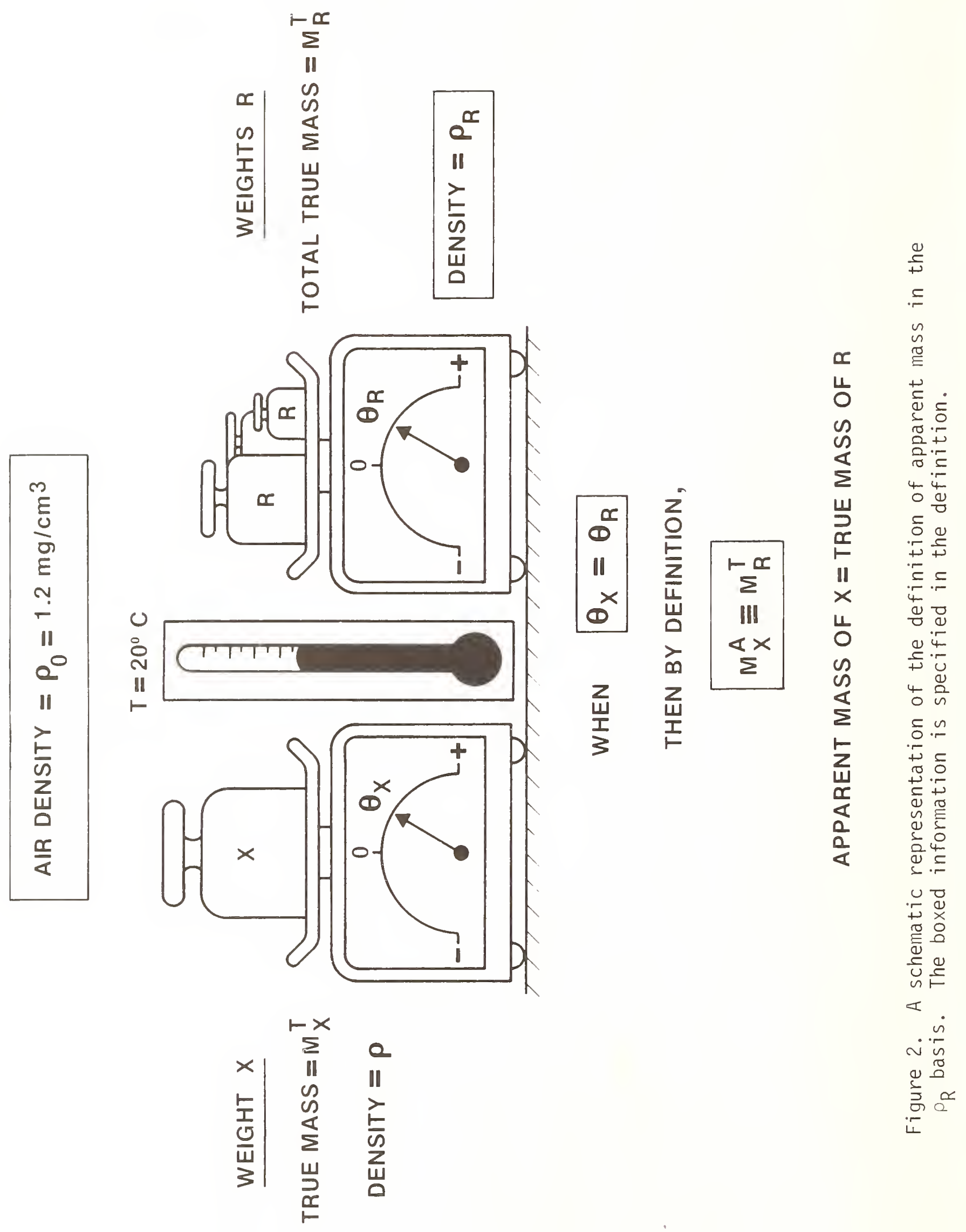




\subsubsection{Example}

Using the ideal balance described earlier, we wish to measure the mass of an unknown weight, $X$, in terms of standard weights, $S$, of known apparent masses $M_{S}^{A_{1}} S$. During the actual measurement, done in air of temperature $t$ and air density $\rho_{A}$, it was found that a summation of standards produced the same reading on the balance as the unknown mass.

Under what conditions can we set

$$
M_{X}^{A} \approx M_{S}^{A} \quad ?
$$

Solution:

Starting with the balance equation for two masses (eq. (10)).

$$
M_{1}-M_{2}=k \Delta \theta+\rho_{A}\left(V_{1}-V_{2}\right)
$$

we have

$$
\begin{aligned}
M_{X}^{T}-M_{S}^{T} & =k\left(\theta_{X}-\theta_{S}\right)+\rho_{A}\left(V_{X}-V_{S}\right) \\
& =\rho_{A}\left(V_{X}-V_{S}\right)
\end{aligned}
$$

since the measurement yielded $\theta_{X}=\theta_{S}$.

Finally, we can write this as

$$
M_{X}^{T}-M_{S}^{T}=\rho_{A} \Delta V
$$

Similarly, we have for the apparent masses, using eq. (11):

$$
\begin{aligned}
M_{X}^{A}-M_{S}^{A} & =\frac{M_{X}^{T}\left(1-\rho_{0} / \rho_{X}\right)}{\left(1-\rho_{0} / \rho_{R}\right)}-\frac{M_{S}^{T}\left(1-\rho_{0} / \rho_{S}\right)}{\left(1-\rho_{0} / \rho_{R}\right)} \\
& =\frac{\left(M_{X}^{T}-M_{S}^{T}\right)-\frac{M_{X}^{T}}{\rho_{X}} \rho_{0}+\frac{M_{S}^{T}}{\rho_{S}} \rho_{0}}{\left(1-\rho_{0} / \rho_{R}\right)}
\end{aligned}
$$

By setting

$$
\frac{M_{X}^{T}}{\rho_{X}}=V_{X 0}, \frac{M_{S}^{T}}{\rho_{S}}=V_{S O} \text { at } 20{ }^{\circ} \mathrm{C}
$$

we simplify to

$$
M_{X}^{A}-M_{S}^{A}=\frac{\left(M_{X}^{T}-M_{S}^{T}\right)-\rho_{0}\left(V_{X_{0}}-V_{S O}\right)}{\left(1-\rho_{0} / \rho_{R}\right)}
$$


Substituting for $M_{X}^{\top}-M_{S}^{\top}$ from (El), we have

$$
M_{X}^{A}-M_{S}^{A}=\frac{\rho_{A}\left(V_{X}-V_{S}\right)-\rho_{0}\left(V_{X O}-V_{S O}\right)}{\left(1-\rho_{0} / \rho_{R}\right)} .
$$

Volumes have a slight temperature dependence but if the temperature of the measurement is close to $20{ }^{\circ} \mathrm{C}$ (generally true in the laboratory), then

$$
v_{x} \approx v_{x O}
$$

and

$$
\mathrm{V}_{\mathrm{S}} \approx \mathrm{V}_{\mathrm{SO}}
$$

so that (E3) reduces to

$$
M_{X}^{A}-M_{S}^{A}=\frac{\left(\rho_{A}-\rho_{0}\right)\left(V_{X}-V_{S}\right)}{\left(1-\rho_{0} / \rho_{R}\right)}=\frac{\Delta \rho \Delta V}{\left(1-\rho_{0} / \rho_{R}\right)} .
$$

The term $1 /\left(1-\rho_{0} / \rho_{R}\right)$ can be approximated by 1 for the following reason: Via a Taylor expansion

$$
\frac{1}{1-\rho_{0} / \rho_{R}}=1+\frac{\rho_{0}}{\rho_{R}}+\left(\frac{\rho_{0}}{\rho_{R}}\right)^{2}+\left(\frac{\rho_{0}}{\rho_{R}}\right)^{3}+\ldots
$$

Now since $\rho_{0}$ is the density of air and $\rho_{R}$ is the density of a reference metal, we have

$$
\frac{\rho_{0}}{\rho_{R}}<10^{-3}
$$

so that

$$
\frac{\Delta \rho \Delta V}{\left(1-\rho_{0} / \rho_{R}\right)}=\Delta \rho \Delta V+\Delta p \Delta V\left(\rho_{0} / \rho_{R}\right)+\cdots
$$

and since $\triangle \rho \Delta V$ is already a small number, a correction of $10^{-3}$ (or 0.1 percent) is insignificant. Hence only the first term has to be retained. This argument is important in that it is used time and again in estimating buoyancy corrections.

With these approximations, we finally arrive at the equation for apparent mass differences

$$
M_{X}^{A}-M_{S}^{A}=\Delta \rho \Delta V .
$$


We now would like to find the conditions for which

$$
M_{X}^{A} \approx M_{S}^{A}
$$

This implies that $\Delta \rho \Delta V \approx 0$.

When one encounters such a relation, one must always decide its precise meaning. In this particular case the meaning is: $\Delta \rho \Delta V$ is smaller than the acceptable uncertainty of $M_{X}^{A}$.

For example, suppose $M_{X}^{A}$ needs to be known to one part per million. Then

really means that

$$
\Delta \rho \Delta V \approx 0
$$

$$
\left|\frac{\Delta \Delta \Delta V}{M_{X}^{A}}\right|<10^{-6} .
$$

But we already know that $M_{X}^{A}$ does not differ from $M_{X}^{\top}$ by more than a fraction of a percent (see comments after eq. (11)). We can therefore approximate

$$
\rho_{x}=\frac{M_{x}^{\top}}{v_{x}}
$$

by

$$
\rho_{x} \simeq \frac{M_{x}^{A}}{v_{x}}
$$

Now (E6) becomes

$$
\left|\frac{\Delta \rho}{\rho_{X}}\right|\left|\frac{\Delta V}{V_{X}}\right|<10^{-6} .
$$

The volume ratio $\Delta V / V_{X}$ can be written as

$$
\frac{\Delta V}{V_{X}}=\frac{V_{X}-V_{S}}{V_{X}} .
$$

In the case of different alloys of stainless steel (SS), $V_{X}$ will be close to $V_{S}$, so that

$$
\left|\frac{\Delta V}{V_{x}}\right|<10^{-1} .
$$


We now have

$$
\left|\frac{\Delta \rho}{\rho_{X}}\right| \times 10^{-1}<10^{-6} .
$$

The density ratio can be written as

where

$$
\frac{\Delta \rho}{\rho_{X}}=\frac{\rho_{A}-\rho_{0}}{\rho_{X}}
$$

$$
\rho_{X} \simeq 8.0 \mathrm{~g} / \mathrm{cm}^{3} \text { for } \mathrm{SS}
$$

and

$$
\rho_{0}=1.2 \times 10^{-3} \mathrm{~g} / \mathrm{cm}^{3}
$$

so that

$$
\frac{\Delta \rho}{\rho_{X}}=\frac{\left(\rho_{A}-1.2 \times 10^{-3}\right)}{8.0} .
$$

The inequality $\left(E T^{\prime}\right)$ now reduces to

which is satisfied as long as

$$
\left|\frac{\rho_{A^{-1}}-2 \times 10^{-3}}{8.0}\right| \times 10^{-1}<10^{-6}
$$

$$
1.28 \times 10^{-3} \mathrm{~g} / \mathrm{cm}^{3} \geq \rho_{A} \geq 1.12 \times 10^{-3} \mathrm{~g} / \mathrm{cm}^{3} \text {. }
$$

This indicates that the air density during the measurement can be off by as much as 0.08 from $1.20 \mathrm{mg} / \mathrm{cm}^{3}$ and still satisfy condition (E6).

In contrast, the buoyancy correction for true masses is much more critical. From eq. (ET), we want to satisfy the condition that

$$
\left|\frac{\rho_{A} \Delta V}{M_{X}^{T}}\right|<10^{-6}
$$

or

$$
\left|\frac{\rho_{A}}{\rho_{X}}\right|\left|\frac{\Delta V}{V_{X}}\right|<10^{-6} .
$$

Using the same material as before, we have

$$
\frac{\rho_{\mathrm{A}}}{8.0} \times 10^{-1}<10^{-6}
$$

so that

$$
\rho_{A}<0.08 \times 10^{-3} \mathrm{~g} / \mathrm{cm}^{3} .
$$


Note that in order to keep the desired accuracy, the air density during the measurement can only be ignored if it is very small, i.e. vacuum.

This has been a long example in which several generally useful techniques have been introduced. It is, therefore, worthwhile to look back and summarize what this example also teaches about apparent mass. The most important inference we can draw is that, for true mass comparisons, we almost always need to account for air buoyancy even though we might be able to use an assumed value for the air density (recall that air density is usually constant in a given laboratory to about \pm 3 percent). For apparent mass comparisons, the air buoyancy correction is much less important and can, therefore, sometimes be ignored. Apparent mass works the way it does 3 because the formalism already has an assumed value for air density (i.e. $1.2 \times 10^{-3} \mathrm{~g} / \mathrm{cm}^{3}$ ) built into it. In measurement situations where this assumed value is not accurate enough, the buoyancy term in eq. (E5) becomes important. This eventuality occurs at precisely the same point when $P_{A}$ in eq. (El) can no longer be sufficiently well approximated by $1.2 \times 10^{-3} \mathrm{~g} / \mathrm{cm}^{3}$.

\subsubsection{Reference Materials}

So far we have not yet defined a reference material. At present, two different apparent mass bases are utilized by the NBS. One of them, the older one, is called "normal brass" and was the logical choice when most laboratory weights were made of brass.

Normal brass is defined by

$$
\begin{aligned}
& \rho_{B O}=8.4 \mathrm{~g} / \mathrm{cm}^{3} \text { at } 0{ }^{\circ} \mathrm{C} \\
& \alpha_{B}=5.4 \times 10^{-5} /{ }^{\circ} \mathrm{C}=\text { coefficient of cubical expansion } \\
& \rho_{O}=1.2 \mathrm{mg} / \mathrm{cm}^{3}=\text { air density at } 20{ }^{\circ} \mathrm{C} .
\end{aligned}
$$

These parameters together with the expression for volumetric expansion

$$
v_{B}(t)=V_{B 0}\left[1+\alpha_{B}\left(t-00^{\circ} \mathrm{C}\right)\right]
$$

and the corresponding densicy correction

$$
\rho_{B}(t)=\rho_{B O} /\left[1+\alpha_{B}\left(t-0{ }^{\circ} \mathrm{C}\right)\right]
$$

completely determine the parameters at $20^{\circ} \mathrm{C}$. Note from above that $\rho_{\mathrm{B} 0}$ is given at $0{ }^{\circ} \mathrm{C}$ and therefore has to be determined for $20^{\circ} \mathrm{C}$ via eq. (13) to yield

$$
\rho_{B}(20)=\frac{8.4}{\left[1+5.4 \times 10^{-5} \times 20\right]}=8.390938 \mathrm{~g} / \mathrm{cm}^{3} \text {. }
$$

The second apparent mass basis is referred to an arbitrary material with the following density:

Once more,

$$
\rho_{0}^{\prime}=8.0 \mathrm{~g} / \mathrm{cm}^{3} \text { at } 20^{\circ} \mathrm{C} \text {. }
$$

$$
\rho_{0}=1.2 \mathrm{mg} / \mathrm{cm}^{3} \text { at } 20{ }^{\circ} \mathrm{C} \text {. }
$$

Note that this basis does not require any expansion coefficient since all parameters are defined at $20^{\circ} \mathrm{C}$. It is therefore comparable to the basis defined for normal brass via eq. (13'). 
It has now become common practice to report masses and their uncertainty in the true mass basis and then quote corrections to nominal values for "normal" brass and for $\rho_{0}^{\prime}=8.0 \mathrm{~g} / \mathrm{cm}^{3}$ bases. Clearly, the latter apparent mass basis is beginning to be preferred by many laboratories since most weights used in a set are made of stainless steel which has a density very close to 8.0 at $20^{\circ} \mathrm{C}$. Thus the correction term in eq. (11) is small, making the apparent mass close to the true mass (see Appendix A.)

We are now in a position to write eq. (11) in the "normal brass" $\left(\rho_{B}\right)$ and in the "8.0" ( $\left.\rho_{0}^{\prime}\right)$ basis. Starting with eq. (11), we have

$$
M_{X}^{A}=M_{X}^{\top} \frac{\left(1-\rho_{0} / \rho_{X}\right)}{\left(1-\rho_{0} / \rho_{R}\right)}
$$

and substituting for $\rho_{R}$ with $\rho_{B}$ from (13), we obtain

$$
M_{X}^{A}=M_{X}^{\top} \frac{\left(1-\rho_{0} / \rho_{X}\right)}{\left(1-\frac{\rho_{0}\left[1+\alpha_{B}\left(t-0{ }^{\circ} \mathrm{C}\right)\right]}{\rho_{B 0}}\right)} .
$$

or

$$
M_{X}^{A}=M_{X}^{\top} \frac{\left(1-\rho_{0} / \rho_{X}\right)}{1-\frac{\rho_{0}}{\rho_{B 0}}\left[1+20 \alpha_{B}\right]}
$$

where the denominator is just a constant equal to 0.9998569886 .

Similarly, we use

$$
\rho_{\mathrm{R}}=\rho_{0}^{\prime}
$$

for the $8.0 \mathrm{~g} / \mathrm{cm}^{3}$ basis to yield

$$
M_{X}^{A}=M_{X}^{\top} \frac{\left(1-\rho_{0} / \rho_{X}\right)}{\left(1-\rho_{0} / \rho_{0}^{\prime}\right)}
$$

where the denominator is once again a constant exactly equal to 0.99985000 . It is interesting to note that apparent masses in the two bases we have examined, eqs. (14) and (15), have a constant ratio

$$
\frac{\left(M^{A}\right)_{\text {BRASS }}}{\left(M^{A}\right)_{8.0}}=1.00000699 .
$$

That is, the apparent masses of the same weight in the two bases differ by only 20.0007 percent. 
Equation (15) indicates a very important point. If the cbject being weighed has a density $\rho_{X}$ equal to $8.0 \mathrm{~g} / \mathrm{cm}^{3}$ (i.e. $\rho_{X}=\rho_{0}^{1}$ ) at $20{ }^{\circ} \mathrm{C}$ then

$$
M_{X}^{A}=M_{X}^{\top} \text {. }
$$

For the brass basis, eq. (14), a similar argument has to be treated with more caution. In this case, if $\rho_{X}$ is equal to the density defined by eq. (13'), then

$$
M_{X}^{A}=M_{X}^{\top} \text {. }
$$

\subsubsection{Reporting Apparent Mass}

Sometimes the apparent mass correction in a given basis is required. This is defined as

$$
\text { Correction }\left(M_{X}^{A}\right) \equiv M_{X}^{A}-N_{X}
$$

where $N_{X}$ is the nominal value of the weight.

2.1.4. Comparing Two Apparent Masses

We now derive an equation similar to (10), appropriate for apparent mass differences. Using eq. (11) we have for mass 1

$$
M_{1}^{A}=\frac{M_{1}^{\top}-\rho_{0} V_{01}}{1-\rho_{0} / \rho_{R}}
$$

where we have used $N_{1}^{\top} / \rho_{1}=V_{01}$.

Similarly we have for mass 2

$$
M_{2}^{A}=\frac{M_{2}^{\top}-\rho_{0} V_{02}}{1-\rho_{0} / \rho_{R}} \text {. }
$$

Subtracting the second from the first yields

$$
M_{1}^{A}-M_{2}^{A}=\frac{M_{1}^{\top}-M_{2}^{\top}-\rho_{0}\left(V_{01}-V_{02}\right)}{1-\rho_{0} / \rho_{R}}
$$

From eq. (10), we have the true mass difference of the two weights

$$
M_{1}^{\top}-M_{2}^{\top}=k \Delta \theta+\rho_{A}\left(V_{1}-V_{2}\right)
$$

so that, upon substitution, we obtain

$$
M_{1}^{A}-M_{2}^{A}=\frac{k \Delta \theta+\rho_{A}\left(V_{1}-V_{2}\right)-\rho_{0}\left(V_{01}-V_{02}\right)}{1-\rho_{0} / \rho_{R}} .
$$

Now, employing eq. (12), we can use 


$$
v_{1}=v_{01}\left[1+\alpha_{1}\left(t-t_{0}\right)\right]
$$

and

$$
v_{2}=v_{02}\left[1+\alpha_{2}\left(t-t_{0}\right)\right]
$$

where $V_{01}$ and $V_{02}$ are the respective volumes at $t_{0}=20^{\circ} \mathrm{C}$.

Substituting for $V_{1}$ and $V_{2}$ into (17) yields

$$
M_{1}^{A}-M_{2}^{A}=k \Delta \theta-\rho_{0}\left(V_{01}-V_{02}\right)+\rho_{A}\left[V_{01}+V_{01} \alpha_{1}(t-20)-V_{02}-V_{02} \alpha_{2}(t-20)\right]
$$

where we have set

$$
t_{0}=20^{\circ} \mathrm{C}
$$

and have approximated $\left(1-\rho_{0} / \rho_{R}\right)^{-1}$ by 1 (see derivation of $\left.(E 5)\right)$.

Simplifying further gives

$$
M_{1}^{A}-M_{2}^{A}=k \Delta \theta+\left(\rho_{A}-\rho_{0}\right)\left(V_{01}-V_{02}\right)+\rho_{A}\left[(t-20)\left(V_{01} \alpha_{1}-V_{02} \alpha_{2}\right)\right] .
$$

Equation (18) is a fully corrected formula for calculating mass differences in the apparent mass basis, except for the one approximation mentioned above. At this point it is worthwhile to consider a specific example in order to get a feeling for the magnitude of the terms in eq. (18). Assume that two weights of nominal value $2 \mathrm{~g}$ have the following properties:

also,

$$
\begin{aligned}
& v_{01}=0.2564 \mathrm{~cm}^{3} \\
& v_{02}=0.1884 \mathrm{~cm}^{3} \\
& \alpha_{1} \approx \alpha_{2}=4.5 \times 10^{-5} /{ }^{\circ} \mathrm{C} ;
\end{aligned}
$$$$
\rho_{A}=1.17 \mathrm{mg} / \mathrm{cm}^{3}
$$$$
\rho_{0}=1.2 \mathrm{mg} / \mathrm{cm}^{3} \text {. }
$$

We then find that

$$
\left(\rho_{A}-\rho_{0}\right)\left(V_{01}-V_{02}\right)=-0.03 \times 0.068=-2.04 \times 10^{-3} \mathrm{mg}
$$

and

$$
\begin{aligned}
\rho_{A}(t-20)\left(v_{01}{ }^{\alpha_{1}}-v_{02}{ }^{\alpha}\right) & =1.17(t-20) \times 3.06 \times 10^{-6} \\
& =\left(3.58 \times 10^{-6}\right)(t-20) .
\end{aligned}
$$

Considering an extreme condition such that $t$ is $10^{\circ} \mathrm{C}$, we have for the latter term

$$
=-3.58 \times 10^{-5} \mathrm{mg} \text {. }
$$

Combining this term with the first we have

$$
-2.04 \times 10^{-3}-0.0358 \times 10^{-3}=-2.08 \times 10^{-3} \mathrm{mg}
$$


so that the second term (under extreme conditions) contributes only $0.04 \mu \mathrm{g}$ to the overall buoyancy correction. Usually the $(t-20)$ term is of the order of $0{ }^{\circ} \mathrm{C}$ to $4{ }^{\circ} \mathrm{C}$ so that the effect of the second term on the overall buoyancy correction of the above weights is even less. Hence in most cases the second term can be safely ignored and eq. (18) can be approximated by

$$
M_{1}^{A}-M_{2}^{A}=k \Delta \theta+\left(\rho_{A}-\rho_{0}\right)\left(V_{01}-V_{02}\right) \text {. }
$$

For true masses we start with eq. (10) and write

$$
M_{1}^{\top}-M_{2}^{\top}=k \Delta \theta+\rho_{A}\left(V_{1}-V_{2}\right) \text {. }
$$

Employing the same volume expansion formulae used for the apparent masses (after eq. (17)), we derive

$$
\begin{gathered}
M_{1}^{\top}-M_{2}^{\top}=k \Delta \theta+\rho_{A}\left(V_{01}-V_{02}\right)+\rho_{A}\left(t-t_{0}\right) \\
x\left(V_{01} \alpha_{1}-V_{02} \alpha_{2}\right) .
\end{gathered}
$$

Once again the second term is very small compared to the first (see arguments above) and the relation can usually be approximated by

$$
M_{1}^{\top}-M_{2}^{\top}=k \Delta \theta+\rho_{A}\left(V_{01}-V_{02}\right) \text {. }
$$

A quick comparison between eqs. (19a) and (19b) shows that the apparent mass difference has a correction term which contains the factor $\left(\rho_{A}-\rho_{0}\right)$ whereas for true masses the corresponding factor is $\rho A$. This then shows that the former correction is much smaller. In both cases the unknown parameter is $\rho_{A}$.

\subsubsection{Sources of Error in Buoyancy Corrections}

It is important to determine the sources of error in buoyancy corrections in order to make the best measurements possible with the least amount of effort and expense. Let us look back to eqs. (19a) and (19b) and write the most important buoyancy terms:

True Mass

$\rho_{A}\left(V_{01}-V_{02}\right)$
Apparent Mass

$$
\left(\rho_{A}-\rho_{0}\right)\left(V_{01}-V_{02}\right)
$$

Since $\rho_{0}$ is defined exactly, uncertainties in the mass difference measurements arising from buoyancy corrections are due to errors in $\rho_{A}, V_{01}$, and $V_{02}$. Let us denote the uncertainty in a quantity by a preceding $\delta$ ( $i . e$. , the uncertainty in $\rho_{A}$ is $\left.\delta \rho_{A}\right)$. Then, from eqs. (19a) and (19b) the sources of uncertainty in the buoyancy corrections are

True Mass

$$
\begin{aligned}
& \delta \rho_{A}\left(V_{01}-V_{02}\right) \\
& \delta V_{01} \rho_{A} \\
& \delta V_{02} \rho_{A}
\end{aligned}
$$

Apparent Mass

$$
\begin{aligned}
& \delta \rho_{A}\left(V_{01}-V_{02}\right) \\
& \delta V_{01}\left(\rho_{A}-\rho_{0}\right) \\
& \delta V_{02}\left(\rho_{A}-\rho_{0}\right)
\end{aligned}
$$

where we will assume that $\delta V_{01}, \delta V_{02}$, and $\delta \rho_{A}$ are uncorrelated. 
In the calculation of true mass differences, uncertainty in the volume of the masses is much more important than it is for differences in apparent mass as can be seen by comparing similar terms:

$$
\left(\delta V_{01}+\delta V_{02}\right) \rho_{A}>\left(\delta V_{01}+\delta V_{02}\right)\left(\rho_{A}-\rho_{0}\right) .
$$

Also we see that any uncertainty in $V_{01}$ or $V_{02}$ will lead to systematic uncertainty in mass measurements (even if $v_{01}=v_{02}$ ).

Example:

Two weights produce the same reading on an ideal balance in air of $\rho_{A}=1.17 \mathrm{mg} / \mathrm{cm}^{3}$ and $t=20^{\circ} \mathrm{C}$. Both weights are said to be of density $7.89 \mathrm{~g} / \mathrm{cm}^{3}$. Find

1) their true mass difference

2) their apparent mass difference against $\rho_{0}^{\prime}=8.0 \mathrm{~g} / \mathrm{cm}^{3}$

3 ) the errors in these numbers due to uncertainty in the volumes.

The weights are marked " $1 \mathrm{~kg} "$.

Answers:

1) From eq. (19b)

$$
M_{1}^{\top}\left(1-\frac{\rho_{A}}{7.89}\right)-M_{2}^{\top}\left(1-\frac{\rho_{A}}{7.89}\right)=k \cdot \Delta \theta=0
$$

because $\Delta \theta$ is given as 0 .

Hence $M_{1}^{\top}-M_{2}^{\top}=0$.

2) From eq. (19a)

$$
M_{1}^{A}-M_{2}^{A}=\left(\rho_{A}-\rho_{0}\right)\left(V_{01}-V_{02}\right)+k \Delta \theta=0
$$

since $\Delta \theta=0$ and $V_{01}=V_{02}$ (because $M_{1}^{\top}=M_{2}^{\top}$ and $\rho_{01}^{\prime}=\rho_{02}^{\prime}$ ).

3 ) the weights could have come from different lots of metal. Since no uncertainty is given for density, we must assume that

$$
\rho_{0}^{\prime}=7.89 \pm 0.005 \mathrm{~g} / \mathrm{cm}^{3} \text {, }
$$

i.e. the density is uncertain in the first unreported decimal place.

As a worst case, let

$$
\rho_{01}^{\prime}=7.895 \text { and } \rho_{02}^{\prime}=7.885 \text {. }
$$

Then 


$$
\begin{aligned}
\left|\delta\left(M_{7}^{\top}-M_{2}^{\top}\right)\right| & \simeq \rho_{A}\left(\frac{M_{2}^{\top}}{7.885}-\frac{M_{1}^{\top}}{7.895}\right) \simeq \rho_{A} M_{1}^{\top}\left(\frac{1}{7.885}-\frac{1}{7.895}\right) \\
& \simeq 0.00117 \times 1000\left(\frac{1}{7.885}-\frac{1}{7.895}\right) \simeq 0.270 \mathrm{mg} \\
\left|\delta\left(M_{1}^{A}-M_{2}^{A}\right)\right| & \simeq 0.00003 \times 1000\left(\frac{1}{7.885}-\frac{1}{7.895}\right) \simeq 0.005 \mathrm{mg}
\end{aligned}
$$

Note that the nominal mass value of each weight is accurate enough to use in estimating the uncertainties in question.

CAUTION: In calibration certificates, it is normal practice not to include in the assignment of systematic errors any contribution from volume uncertainties: The volumes, usually calculated from densities provided by the weight manufacturers, are assumed to be without error. For the most critical work, volumes of individual weights must actually be measured. (Since 1982, NBS has been routinely measuring the volume of single-piece 1-kilogram weights which require the best possible mass calibration.)

We have yet to examine the effects on mass measurements of uncertainty in $\rho_{A}$. For mass differences, we have shown above that the errors are approximately $\delta \rho_{A}\left(V_{01}-V_{02}\right)$. Therefore, unless $V_{01}=V_{02}$, we mst be concerned with uncertainty in $\rho_{A}$. The calculation of $\rho_{A}$ along with an analysis of the attendant uncertainties are presented in the next section.

\subsection{Air Density}

We have seen from the derivations in Section 2.1 that the correction to the mass determination requires the precise knowledge of the air density $\left(\rho_{A}\right)$ if the weighing is done in an air environment. (We will assume from now on that we are always working in air.) The buoyancy correction in eq. (19b) requires $\rho_{A}$ for true masses, whereas for apparent masses we require $\left(\rho_{A}-\rho_{0}\right)$ in eq. (19a). It should be remembered that $\rho_{0}$ is defined as $\rho_{0}=1.2 \mathrm{mg} / \mathrm{cm}^{3}$ which is the approximate value for $20{ }^{\circ} \mathrm{C}$ with 1 atmosphere of pressure with $50 \%$ relative humidity.

The usual approach to determining $\rho_{A}$ is to calculate the density of air based on an "equation-of-state." To use this equation, one must supply ambient values for temperature, barometric pressure, and relative humidity. For extremely exacting work, the concentration of carbon dioxide in the laboratory ambient is also required. Appendix $B$ contains a derivation of the equation-of-state based on the work of Jones [2]. This formulation is close to those which are found in handbooks or older references but has three unique virtues: 
1. It is based on the most recent reference data available.

2. It has been derived in such a way that uncertainties associated with the equation itself are known and stated.

3. Virtually the same equation has been endorsed for international use by the Consultative Committee for Mass of the International Committee of Weights and Measures (C.I.P.M.).3

The interested reader is urged to consult Appendix B and especially Ref. [2] for the complete derivation. We state here that 4

$$
\rho_{A}\left(\frac{\mathrm{mg}}{\mathrm{cm}^{3}}\right)=\frac{0.0034848}{(t+273.15)} \cdot\left(P-0.0037960 \cdot U \cdot \mathrm{e}_{\mathrm{s}}\right)
$$

where

$$
\begin{aligned}
t= & \text { temperature in }{ }^{\circ} \mathrm{C} \\
\mathrm{P}= & \text { barometric pressure in pascals } \\
& (133.322 \mathrm{~Pa}=1 \mathrm{~mm} \mathrm{Hg}) \\
\mathrm{U}= & \text { relative humidity in } \% \\
\mathrm{e}_{\mathrm{S}}= & \text { saturation vapor pressure. } \\
\mathrm{e}_{\mathrm{S}}= & 1.7526 \times 10^{11} \text { exp }(-5315.56 /(273.15+\mathrm{t})) \mathrm{Pa} \\
& \text { is an excellent approximation. }
\end{aligned}
$$

If barometric pressure is measured in $\mathrm{mm} \mathrm{Hg}$, then

$$
\rho_{A}\left(\frac{\mathrm{mg}}{\mathrm{cm}^{3}}\right)=\frac{0.46460}{(t+273.15)}\left(P-0.0037960 \mathrm{U} \cdot \mathrm{e}_{\mathrm{s}}\right)
$$

where $e_{S}=1.3146 \times 10^{9} \exp (-5315.56 /(273.15+t)) \mathrm{mm} \mathrm{Hg}$ is an excel lent approximation.

\subsection{Sensitivity Arguments}

The major correction normally required for mass determination is due to air buoyancy. As is evident from eqs. (19a) and (19b) the only parameter that has to be determined very carefully, aside from the volumes of the weights being compared, is the air density. Equation (20) clearly indicates that three parameters affect the air density, namely humidity, barometric pressure, and temperature. We will see in Section 2.3.5 that an overal1 uncertainty in $\rho_{A}$ of $\sim 0.0030 \mathrm{mg} / \mathrm{cm}^{3}$, i.e. 20.25 percent of $P_{A}$ near sea level, is usualiy acceptable. In Appendix $C$ we see that the total uncertainty in $\rho_{A}$ is the square root of the sum of the squares of the individual uncertainties arising from measurements of humidity, temperature and pressure, ( $U$, $t$ and $P$ ). Thus one way of assuring that the total uncertainty of $\rho_{A}$ is within acceptable limits is to make sure that the individual uncertainties from $U$, $t$ and $P$ are each less than $1 / \sqrt{ } 3$ of the total acceptable uncertainty. That is, we set a goal that 3 the contribution to uncertainty in $\rho_{A}$ from $U, P$, or $t$ should be less than $0.0017 \mathrm{mg} / \mathrm{cm}^{3}$. We will see in Section 2.4 that this goal can be met or exceeded with a modest investment in equipment. We now show the relative importance of measurements of humidity, ${ }^{3}$ The CCM recommends that this equation referred to as "Euqation for the determination of the density of moist air (1981): in anticipation of improved reference data. There are some stylistic differences in the CCM version of [2] but the calculated values are completely consistent at meaningful levels of precision.

${ }^{4}$ Equations (20a) and (20b) are somewhat simplified (see Appendix B). Weighing errors due to the simplifications are usually very small and are estimated in Table 5, p. 427 of ref. [2]. 
temperature, and pressure to the uncertainty in air density; we also indicate now accurately these must be measured to achieve our goal of $0.0017 \mathrm{mg} / \mathrm{cm}^{3}$ for each one.

\subsubsection{Humidity}

It is easiest to discuss the effects of humidity (U) via examples. For practical purposes we can consider as standard a condition when $t=20^{\circ} \mathrm{C}, \mathrm{P}=760.00 \mathrm{~mm} \mathrm{Hg}$, and $U=50$ percent.

\section{Example 1}

Consider $t=20^{\circ} \mathrm{C}, P=760.00 \mathrm{~mm} \mathrm{Hg}, U=50$ percent (i.e. standard condition). Using eq. (20b), we find

$$
\rho_{A}=1.2045-0.0053=1.1992 \mathrm{mg} / \mathrm{cm}^{3} \text {. }
$$

We note that the humidity correction, $0.0053 \mathrm{mg} / \mathrm{cm}^{3}$, corresponds to a correction of only 0.44 percent in $\rho_{A}$.

\section{Example 2}

Same conditions as in Example 1 except $t=30^{\circ} \mathrm{C}$. We calculate

$$
\rho_{A}=1.1648-0.0093=1.1555 \mathrm{mg} / \mathrm{cm}^{3}
$$

and the humidity corrections is 20.8 percent of $\rho_{A}$.

\section{Example 3}

Same as Example 1 except $U=60$ percent.

$$
\rho_{A}=1.2045-0.006330=1.1982 \mathrm{mg} / \mathrm{cm}^{3}
$$

so that the humidity correction is 0.5 percent.

Summarizing the results on humidity we can make the following observations:

i. The magnitude of the humidity correction to the air density at standard conditions is 0.4 percent.

ii. By increasing the temperature from $20{ }^{\circ} \mathrm{C}$ to $30{ }^{\circ} \mathrm{C}$, the humidity correction amounts to 0.8 percent of the air density. Thus even under these high temperatures, very unlikely in a temperature-controlled laboratory, the humidity correction term is still quite small.

iii. A change of 10 percent in the relative humidity (i.e. $U=U_{0}+10$ ) changes the air density by only 0.08 percent (i.e. $\frac{1.1992-1.1982}{1.1992}$ ).

We can conclude, therefore, that if we want to know the air density to within $0.0017 \mathrm{mg} / \mathrm{cm}^{3}$ then the relative humidity should be known to \pm 16 percent. (If a safety factor of 4 is required then the relative humidity should be known at the \pm 4 percent level). 


\subsubsection{Temperature}

The effects of temperature on the humidity term were already demonstrated in Example 2. In particular we note that a $10^{\circ} \mathrm{C}$ change, yielded a humidity correction of 0.8 percent.

The most sensitive effect of temperature on calculated air density, however, occurs in the first term

$$
\rho_{A}=\frac{0.46460}{(t+273.15)} P
$$

From Example 1, we note that at standard conditions the first term yields

$$
\bar{\rho}=1.2045 \mathrm{mg} / \mathrm{cm}^{3}
$$

Example 4

$P=760.0 \mathrm{~mm} \mathrm{Hg}, t=21^{\circ} \mathrm{C}$

In this case the first term of eq. (20b) gives

$$
\rho_{A}=1.2004 \mathrm{mg} / \mathrm{cm}^{3}
$$

and $\rho_{A}$ has changed from $\bar{\rho}$ by $0.0041 \mathrm{mg} / \mathrm{cm}^{3}$ or 0.34 percent.

To achieve an uncertainty of $0.0017 \mathrm{mg} / \mathrm{cm}^{3}$, we must measure the temperature accurately to $\approx \pm 0.4^{\circ} \mathrm{C}$ (which reduces to $\pm 0.1{ }^{\circ} \mathrm{C}$ for a safety factor of 4. )

\subsubsection{Pressure}

The arguments for the pressure uncertainty follow those of the temperature. Once again we only have to consider the first term of the density equation

$$
\rho_{A}=\frac{0.46460}{(t+273.15)} P
$$

For standard conditions of $t=20^{\circ} \mathrm{C}$, and $P=760.0 \mathrm{~mm} \mathrm{Hg}$

$$
\bar{\rho}=1.2045 \mathrm{mg} / \mathrm{cm}^{3}
$$

\section{Example 5}

$$
\begin{aligned}
& P=764.00 \mathrm{~mm} \mathrm{Hg} \\
& t=20{ }^{\circ} \mathrm{C}
\end{aligned}
$$

In this case $\rho_{A}=1.2108$ and has changed from $\bar{\rho}$ by 0.53 percent.

Thus in order to know $\rho_{A}$ to $0.0017 \mathrm{mg} / \mathrm{cm}^{3}$ we will need to have accurate barometric measurements to $\pm 1.7 \mathrm{~mm} \mathrm{Hg}$ (which reduces to $\pm 0.3 \mathrm{~mm} \mathrm{Hg}$ for a safety factor of 4).

\subsubsection{Overall Uncertainty of Air Density}

We have seen in eq. (20) that the air density is a function of the temperature, pressure, and humidity 


$$
\rho_{A}=\rho_{A}(t, P, U) \text {. }
$$

To evaluate the overall uncertainty of $\rho_{A}$, we assume uncorrelated errors and develop a final value in Appendix C:

$$
\frac{\delta \rho_{A}}{\rho_{A}} \sim 0.25 \text { percent } .
$$

Note in particular that this value was obtained using the errors established in Sections 2.3.1, 2.3.2, and 2.3.3 of this section (with no safety factor).

\subsubsection{Effects of Small Changes in Air Density on the Mass Value}

Having established the sensitivities of $U, t, P$ on $\rho_{A}$, the air density, we can now proceed and see what effect a small change of $\rho_{A}$ has on the mass determination. The arguments can best be carried through with the following example:

Assume that we are comparing the masses of two $1 \mathrm{~kg}$ weights.

$$
\begin{aligned}
& \text { Weight \#1 has density of } 8.4 \mathrm{~g} / \mathrm{cm}^{3} \\
& \text { Weight \#2 has density of } 7.5 \mathrm{~g} / \mathrm{cm}^{3} \\
& \text { Air density } \rho_{A}=1.20 \mathrm{mg} / \mathrm{cm}^{3} \text {. }
\end{aligned}
$$

If the air density is known to an accuracy of 0.25 percent, what uncertainty does this cause in the mass difference calculations?

Answer: We found in Section 2.1.5 that the uncertainty for true as well as apparent mass was given as

$$
\begin{aligned}
\text { uncertainty } & = \pm \delta \rho_{A}\left(V_{01}-V_{02}\right) \\
& = \pm \delta \rho_{A} N\left(\frac{1}{\rho_{1}}-\frac{1}{\rho_{2}}\right)
\end{aligned}
$$

where we have approximated the volumes by

$$
v_{1} \sim N / \rho_{1} ; V_{2} \sim N / \rho_{2}
$$

with $N$ being the nominal mass of each weight.

Substituting numerical values, yields

$$
\begin{aligned}
\text { Uncertainty } & = \pm 0.0025 \times \rho_{A} \times 1000\left(\frac{1}{8.4}-\frac{1}{7.5}\right) \\
& = \pm 0.0025 \times 1.2 \times 1000\left(\frac{1}{8.4}-\frac{1}{7.5}\right) \\
& = \pm 0.043 \mathrm{mg} .
\end{aligned}
$$


This is about one-half the calibration uncertainty limit provided by the NBS for high-quality $1-\mathrm{kg}$ weights. The span 7.5 to $8.4 \mathrm{~g} / \mathrm{cm}^{3}$ includes the densities of $1-\mathrm{kg}$ weights used in routine fine work.

It is usually true that the most stringent requirements placed on buoyancy measurements are in the precise comparison of kilograms. An uncertainty in $\rho_{A}$ of 0.25 percent is usually sufficient even in this case.

CAUTION: Some weights are manufactured for purposes other than general mass metrology. For instance, a scientist may have a $1-\mathrm{kg}$ aluminum weight which needs the best calibration possible. In such special situations, the requirements and limitations placed on buoyancy work must be examined on a case-by-case basis.

\subsection{Measuring Equipment for Air Density}

The previous section yielded information on the accuracies required for the three parameters needed for the air density. In this section we discuss briefly the types of equipment required to meet our accuracy goals.

\subsubsection{Humidity}

This parameter is usually measured with a psychrometer or hygrometer. Several commercial instruments can provide accuracies of $\pm 2 \%$ relative humidity over a wide range such as $10-80 \%$. The calibration of such instruments can be verified at fixed points by the user by means of an in-laboratory calibration station. Since an accuraçy of $\pm 16 \%$ (or $\pm 4 \%$ using a "safety factor of 4 ") is required for mass metrology, these instruments are more than adequate.

\subsubsection{Temperature}

An accuracy of $\pm 0.4{ }^{\circ} \mathrm{C}$ (or $\pm 0.1^{\circ} \mathrm{C}$ for a "safety factor of $4^{\prime \prime}$ ) is required. Simple, mercury-filled, glass thermometers can provide reading accuracies up to \pm 0.1 ${ }^{\circ} \mathrm{C}$. More elaborate units can yield $\pm 0.01{ }^{\circ} \mathrm{C}$.

The user should be very careful in taking temperature readings. Generally, the physical presence of the metrologist tends to warm up the balance with respect to the rest of the room. It is therefore essential that, no matter what form of thermometer is used, its sensor be placed as close as possible to the balance pan. Also, every attempt should be made to ensure that objects being weighed are in thermal equilibrium with the balance. The latter requirement is especially important when comparing weights of large surface area or of different geometries.

\subsubsection{Barometric Pressure}

The required accuracy is $\pm 1.1 \mathrm{~mm} \mathrm{Hg}$ (or $\pm 0.3 \mathrm{~mm} \mathrm{Hg}$ for a "safety factor of 4"). One can obtain mercury manometers or aneroid barometers with reading accuracies of $\pm 0.05 \mathrm{~mm} \mathrm{Hg}$. Since this is sufficient for usual mass calibrations, one does not have to push for higher accuracy instruments.

Present technology can provide defining instruments with overall uncertainty of $\pm 0.01 \mathrm{~mm} \mathrm{Hg}$. Since such devices are the most accurate on the market, they are generally used in calibration of other manometers or aneroid barometers which consequently would have greater uncertainty. 
Many laboratories possess either Fortin-type or aneroid barometers. These require calibration against some defining instrument--such as a mercury manometer or a piston gage; but such defining instruments are costly and may not be available in the laboratory. On the other hand, aneroid and Fortin-type barometers are best calibrated in place.

A solution to this problem has recently been demonstrated [9]. Two weights of well-known and nearly-equal mass but having very different volumes are compared in the laboratory on a sensitive balance. The measured difference in balance readings between these two weights, $k \Delta \theta$, determines the air density in the balance enclosure via eq. (9). The air density is also calculated from eq. (20) using a calibrated thermometer, a calibrated hygrometer, and the uncalibrated barometer. The difference in the two measurements of air density serves to calibrate the barometer. Calibration uncertainties of less than $1 \mathrm{~mm} \mathrm{Hig}$ (three standard deviations plus known systematic errors) have been demonstrated.

In summary, we note that all the requirements for air density measurements can be met with presently available instrumentation. If, however, the accuracy of \pm 0.25 percent for $\rho_{A}$ has to be improved, then the limiting instrument will be the pressure gage. (It does not make sense to improve temperature and humidity resolution by an order of magnitude, if the pressure cannot be read with higher precision.) 


\section{WEIGHING METHODS}

Until this point, we have assumed that all mass comparisons have been carried out on the idealized balance defined in Section 2. In this section we develop the means of evaluating the constant, $k$ of eq. (8). We also extend our analysis to actual balances currently in use. Just as one need not be a mechanical engineer to drive a car, so one need not have a detailed knowledge of balance design in order to carry out successful mass measurements. Nevertheless, a general knowledge of balance design is often useful. The review paper by Schoonover [10] provides an excellent introduction to this subject.

The idealized balance of Section 2 had no imprecision. Unfortunately, this is not the case for real balances. By saying a balance has imprecision, we mean two distinct contributions to uncertainty:

1) Resolution. A balance observation can be no more certain than one's ability to resolve the least-significant digit of the read-out.

2) Reproducibility. A balance observation can be no more certain than the tendency of the balance to produce identical read-outs under identical conditions. The lack of this property is called "scatter."

For balances used in the best metrology, scatter is by far the more important of the two. What we loosely term the scatter of the balance is not entirely intrinsic to the balance. That is, it may depend on the type of table on which the balance is placed, the type of air-conditioning in the laboratory, the skill of the balance operator, etc. We need a measure of the scatter which includes all these effects. Such a measure is provided by the "process standard deviation" $[7,8]$.

The standard deviation for a particular weighing process is easily estimated by repeating the process a number of times. (Ten repetitions are usually sufficient to give a respectable estimate.) Two weights, representative of the weights normally measured on a particular balance, are intercompared $n$ times by one of the methods described below. The $n$ values obtained are $a_{1}, a_{2}, a_{3}, \ldots, a_{n-1}, a_{n}$. The average of the $n$ values is

$$
\bar{a}=\frac{\sum_{i=1}^{n} a_{j}}{n}
$$

and the standard deviation $(\sigma)$ of the balance is estimated to be

$$
s=\left(\frac{\sum_{j=1}^{n}\left(a_{i}-\bar{a}\right)^{2}}{n-1}\right)^{1 / 2}
$$

with $s \rightarrow \sigma$ as $n \rightarrow \infty$. (The estimate $s$ approaches the actual standard deviation $\sigma$ as $n$ is made larger.)

The standard deviation should be estimated in this way at regular intervals in order to spot trends or shifts in the evolution of $s$ with time. A slow rise in $s$ could signal, for example, a slow deterioration of the balance. An abrupt shift could signal a problem with the balance, a change in the air-conditioning system of the laboratory, or some other problem which requires attention. 
Before actually discussing various types of weighing procedures, we have to define some terms required in the descriptions. The following symbols will be utilized.

$M=$ any unknown weight with true mass $M^{\top}$ whose value has to be determined

$C=$ a counterpoise (or counterweight) with true mass $C^{\top}$.

This weight is usually required on beam balances. It can be of any stable shape, form and composition. However, it has to be adjusted so that the scale (balance) does not deflect past its maximum allowable value when loaded with a weight. This mass appears in equations which are combined in such a way that $C^{\top}$ drops out of the final result.

$S=$ a standard weight with true mass $S^{\top}$.

This is a known mass against which the unknown is measured. It is always assumed that in any process $S^{\top}$ is known and is traceable to the NBS. In the following description $S$ can be of a single unit or can be made up of

$$
S=\sum_{i=1}^{n} S_{i} \quad \text { such that the sum } S^{\top} \text { roughly equals } M^{\top} \text { or } C^{\top} \text {. }
$$

$\Delta=$ a sensitivity weight with true mass $\Delta^{\top}$

This mass is usually of very small magnitude. In principle it is only used to measure the deflection of the scale per unit mass. This mass is very critical. It must be well calibrated and traceable to the NBS, although such calibration is a relatively simple matter.

Of the various weighing procedures, we will mention Direct Weighing, DirectReading Weighing, and discuss Substitution and Transposition Methods.

\subsection{Direct Weighing}

This type of weighing is seldom used in the mass calibration program. It requires the use of an equal-arm balance. Essentially, the reading steps are as follows:

1. Release brake and take null reading with both pans empty.

2. Load one pan with $M$ and balance with $S$ masses on the opposing pan until the null position is once again reached.

We have as final result, if buoyancy corrections are insignificant,

$$
M^{\top}=\sum_{i=1}^{n} S_{i}^{T}
$$




\subsection{Direct-Reading Weighing}

This weighing procedure requires a one-pan balance that reads directly in S.I. mass units or some other commercially recognized unit (e.g. pounds or carats). The procedural steps are as follows:

1. Nul1 the balance without any load on the pan.

2. Place M on pan and record the mass directly.

This method is obviously quite simple but usually is not accurate enough for metrology. It is appropriate for scales most commonly used in stores. Nevertheless, the method is quite similar in principle to that described in Section 3.4.5., below.

\subsection{Substitution Weighing}

This weighing procedure is the usual one employed for both single pan or equalarm balances. The basic idea is the comparison of an unknown mass with a standard mass and a sensitivity mass.

\subsubsection{Single Pan Balance}

Known as "direct-reading analytical balances," they contain built-in weights which are usually manipulated by external knobs or dials. These we will refer to as "dial weights." The remaining balance reading is indicated by the rest point of a moving optical screen or by an automatic electronic display. This part of the balance output is called the "screen reading." Thus each balance indication is the summation of the dial weights and the screen reading. Calibration of the dial weights is unnecessary for the measurements described below. The majority of balance operations outside metrology (e.g., chemistry, metallurgy, etc.) do rely on the accuracy of the built-in dial weights, however. For calibration of these weights the reader is referred to Ref. [11] and Appendix A.

\subsubsection{Single Substitution}

To find the mass difference between two weights of nominally equal value, we will first describe the method of "single substitution". For the moment, let us assume that the weights are matched closely enough so that, when placed on the balance, they both require the same setting of dial weights. Therefore they differ only in their screen readings. If we look into the balance (fig. 3 ), we see that the pan is suspended from a beam which pivots about a very good bearing (the fulcrum, usually a knife on a flat). On the end of the beam opposite to the pan, is a fixed counterpoise C. (There will also be either a servo-motor on this end or a dashpot to damp the beam oscillations. These details are unimportant to our derivation.) Also suspended from the pan-end of the beam are the built-in dial weights. Thus when an object is placed on the pan, dial weights are removed so that the force of the total assembly on the pan side (object being weighed plus remaining dial weights) balances the counterweight as closely as possible. The remaining imbalance causes the beam to rock slightly out of its horizontal position. The angle of tilt is proportional to the remaining force imbalance. In a mechanical balance, this angle is directly proportional to the screen readings. In a servo-controlled balance, a motor is used to drive the beam back to horizontal. The screen readings are then proportional to the average force generated by the motor. 


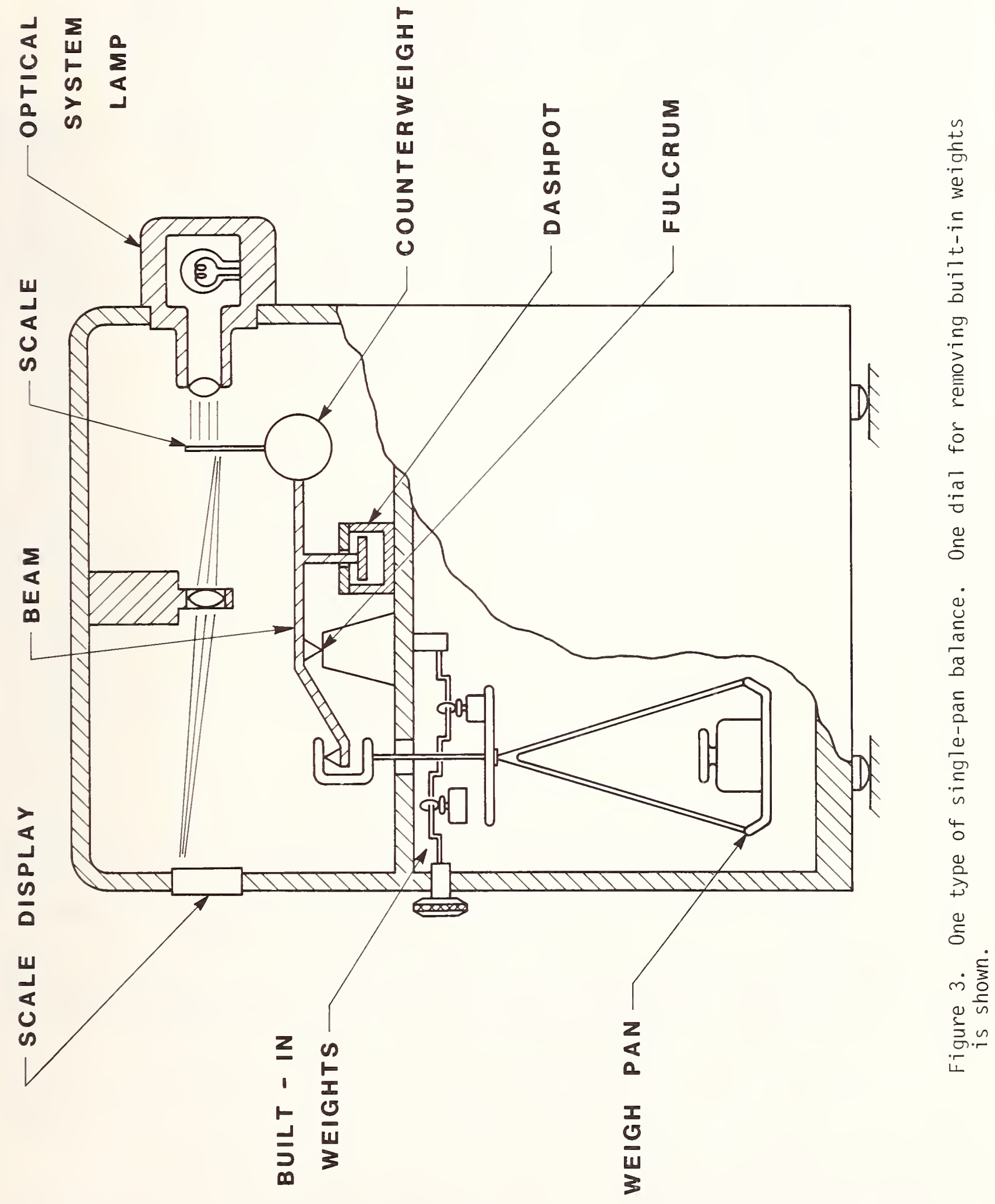


In the balances just described, we can define the following quantities:

$\Sigma D_{i}^{\top}=$ the true mass of the dial weights removed

$\Sigma V_{i}=$ the volume of the dial weights removed

$\theta_{1}=$ the screen reading

$\theta_{0}=$ the screen reading when the pan is empty and the dial weights are replaced (this was always zero for the ideal balance of Section 2)

$k$ = the proportionality constant between screen reading and mass.

(Recall the previous definitions for $M, S, \Delta_{.}$) We also recall from eq. (8) that for two weights, $M_{1}$ and $M_{2}$ with volumes $V_{1}$ and $V_{2}$, the weighing equation can be written as (using true masses)

$$
\bar{g}\left(M_{1}^{\top}-\rho_{m} V_{1}\right)=\bar{g}\left(M_{2}^{\top}-\rho_{m} V_{2}\right)+\bar{g} k(\Delta \theta) .
$$

With

and setting

$$
\begin{gathered}
\rho_{m}=\rho_{A}=\text { air density; } \\
(\Delta \theta)=\theta_{1}-\theta_{2} ;
\end{gathered}
$$

we arrive $a t^{5}$

$$
\begin{aligned}
& M_{2}^{\top}=\Sigma D_{i}^{\top} \\
& V_{2}=\Sigma V_{i}
\end{aligned}
$$

$$
\bar{g}\left(M_{1}^{\top}-\rho_{A} V_{1}\right)=\left(\Sigma D_{i}^{\top}-\rho_{A} \Sigma V_{i}\right) \bar{g}+\bar{g} k\left(\theta_{1}-\theta_{0}\right)
$$

where $\theta_{0} \equiv \theta_{2}$ in this notation.

The following observations can be made:

- The counterpoise weight does not_enter into the final result.

- The gravitational acceleration, $\bar{g}$, can be cancelled from both sides of the above balance equation.

- The above equation is identical to that for the true mass difference between weight \#1 and the removed dial weights (see Appendix A).

In single substitution, we compare a weight $M$ with a standard weight $S$. Either $M$ or S may actually be a summation of weight pieces. A small calibration weight, sensitivity weight $\Delta$, is also required.

${ }^{5} \mathrm{~A}$ full derivation would involve a balance equation for each weighing operation (i.e. the pan unloaded and the pan loaded). These equations would each involve the counterpoise C. Combining these relations would then give the desired result. 
The steps in single substitution are:

1) Load $M$ and record screen reading $\theta_{1}$

2) Remove $M$

3) Load $S$ and record screen reading $\theta_{2}$

4) Add $\Delta$ to $S$ and record screen reading $\theta_{3}$

In general we adjust $S$ and choose $\Delta$ so that

- The same dial weights are used in steps 1,3 , and 4 .

- $\left|\theta_{3}-\theta_{2}\right|$ is at least four times as large as $\left|\theta_{2}-\theta_{1}\right|$

- $\left(\theta_{3}-\theta_{2}\right) \sim 1 / 5$ the total range of the screen.

From the above steps 1,3 and 4 , we have the following relations:

$$
\begin{aligned}
& k \theta_{1} \doteq\left(M^{\top}-\rho_{A} V_{M}\right)-\left(\Sigma D_{i}^{\top}-\rho_{A} \Sigma V_{i}\right)+k \theta_{0} \\
& k \theta_{2} \doteq\left(S^{\top}-\rho_{A} V_{S}\right)-\left(\Sigma D_{i}^{\top}-\rho_{A} \Sigma V_{i}\right)+k \theta_{0} \\
& k \theta_{3} \doteq\left(S^{\top}-\rho_{A} V_{S}\right)+\left(\Delta^{\top}-\rho_{A} V_{\Delta}\right)-\left(\Sigma D_{i}^{\top}-\rho_{A} \Sigma V_{i}\right)+k \theta_{0} .
\end{aligned}
$$

The symbol $\doteq$ indicates that we are now assuming that some or all of the measured values have a non-negligible uncertainty.

Subtracting (23) from (22) yields

$$
M^{\top}-S^{\top}=\rho_{A}\left(V_{M}-V_{S}\right)+k\left(\theta_{1}-\theta_{2}\right) \text {. }
$$

Subtracting (24) from (23) yields

$$
k=\frac{\Delta^{\top}-\rho_{A} V_{\Delta}}{\left(\theta_{3}-\theta_{2}\right)} \text {. }
$$

Finally, substituting for $k$ from (26) into (25) yields

$$
M^{\top}-S^{\top}=\rho_{A}\left(V_{M}-V_{S}\right)+\frac{\Delta^{\top}-\rho_{A} V_{\Delta}}{\left(\theta_{3}-\theta_{2}\right)}\left(\theta_{1}-\theta_{2}\right) .
$$

Note that this equation is identical to (10), the crucial difference being that we have found the value for the proportionality constant $k$.

One may wonder why $k$ must be evaluated for every measurement if it is a constant. The answer is that for most sensitive balances, $k$ is truly constant only for short periods of time and only over reduced regions of the range of the screen.

In the derivation above we choose $\Delta$ so that $\theta_{3}-\theta_{2}$ is about 20 percent of the screen range (fig. 4). In this reduced region, $k$ will be sufficiently constant, for metrology if one is using well-made balances. 


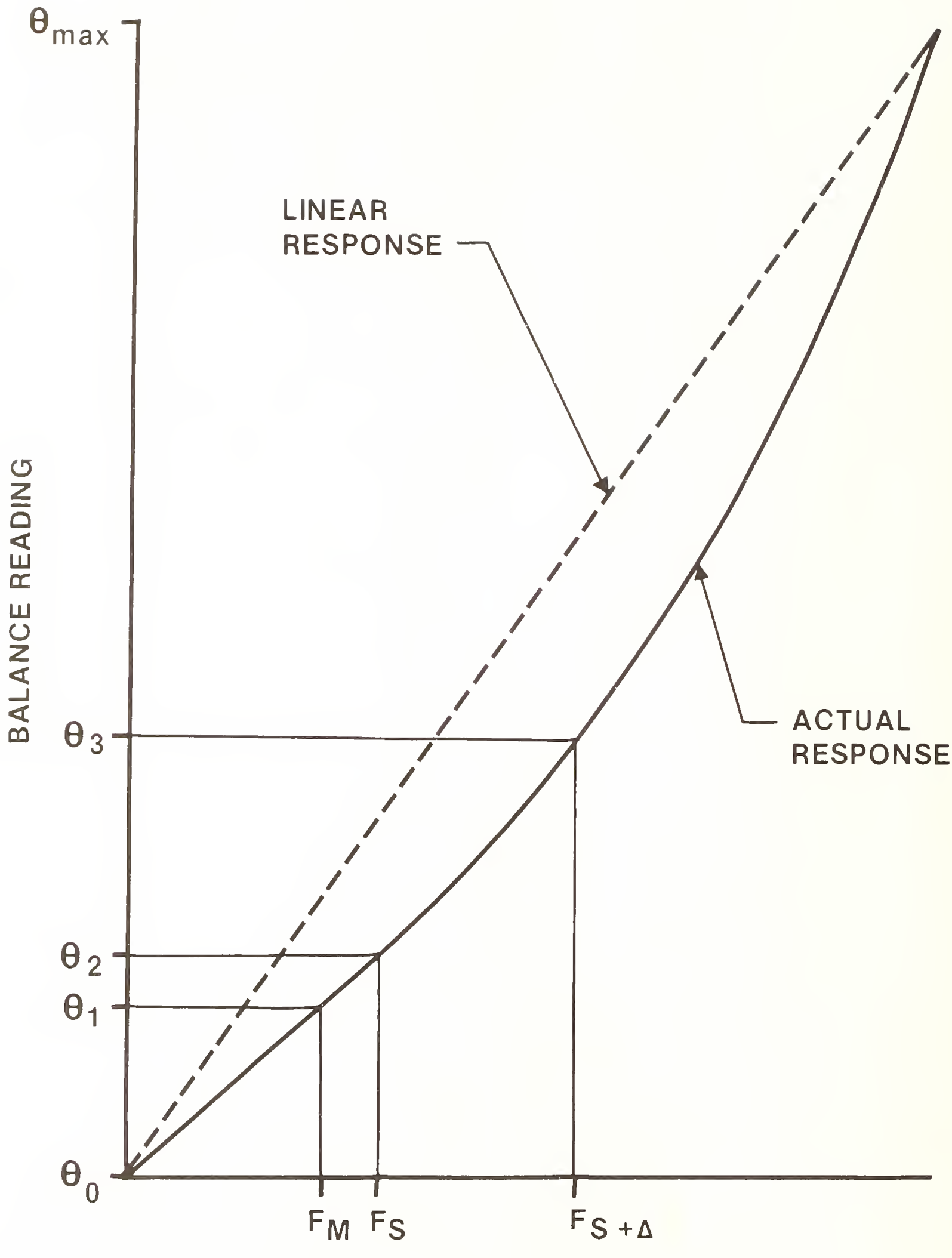

FORCE ON BALANCE

Figure 4. Balance indications as a function of the force on the pan. The response shown is that of the screen when the dial weights are not changed. 


\subsubsection{Double-Substitution}

In the derivation of single-pan substitution given above, we have treated $\theta_{0}$ as a constant. This is the same as assuming that the balance indication does noi drift with time. But this is rarely the case. Usually, however, the zero-drift in the balance will be approximately linear over a short period of time. The method known as single-pan double-substituion has the following two advantages over singlesubstitution:

- linear drifts in the balance zero are eliminated if there is an equal time between successive observations;

- a second estimate of the mass difference between $M$ and $S$ is obtained.

Both benefits are obtained by a single additional measurement:

5) Remove $S$ from pan, leave $\Delta$ where it is

6) Place $M$ on the pan and record secreen reading $\theta_{4}$. The last step gives the additional balance relation

$$
k \theta_{4} \doteq\left(M^{\top}-\rho_{A} V_{M}\right)+\left(\Delta^{\top}-\rho_{A} V_{\Delta}\right)-\left(\Sigma D_{j}^{\top}-\rho_{A} \Sigma V_{j}\right)+k \theta_{0}
$$

Substracting (24) from (28) yields

$$
M^{\top}-S^{\top}=\rho_{A}\left(V_{M}-V_{S}\right)+k\left(\theta_{4}-\theta_{3}\right) \text {. }
$$

Substituting for $k$ from eq $(26)$ then gives

$$
M^{\top}-S^{\top}=\rho_{A}\left(V_{M}-V_{S}\right)+\frac{\left(\Delta^{\top}-\rho_{A} V_{\Delta}\right)}{\left(\theta_{3}-\theta_{2}\right)}\left(\theta_{4}-\theta_{3}\right) \text {. }
$$

Finally, by adding (29) and (27) we obtain

$$
M^{\top}-S^{\top}=\rho_{A}\left(V_{M}-V_{S}\right)+\frac{\left(\Delta^{\top}-\rho_{A} V_{\Delta}\right)}{2\left(\theta_{3}-\theta_{2}\right)}\left(\theta_{1}-\theta_{2}-\theta_{3}+\theta_{4}\right) \text {. }
$$

One might wonder how eq. (30) would have to be changed if $\theta_{0}$ were drifting linearly with time instead of being constant. The answer is: the term $\left(\theta_{1}-\theta_{2}-\theta_{3}+\theta_{4}\right)$ would still be strictly correct; the term $\left(\theta_{3}-\theta_{2}\right)$ would not be strictly correct. However, provided that the drift among readings is small compared to $\left(\theta_{3}-\theta_{2}\right)$, the error from this source will be negligible. This will in actuality always be the case. If it were not, the balance would be drifting so badly that no stable readings could be obtained. If instead of $\left(\theta_{3}-\theta_{2}\right)$ one substitutes the term $\left(3 \theta_{3}-3 \theta_{2}+\theta_{1}-\theta_{4}\right) / 2$, linear drift in the sensitivity calculation will be removed [12]. This more complicated formulation is generally used when data are being analyzed by digitalcomputer software. 
CAUTION: If $\theta_{1}$ or $\theta_{2}$ is very close to zero, balance drift may cause readings to become negative or to go off scale. To avoid this problem, a small, uncalibrated "tare weight" can be added to the pan during all measurements. Mathematically, this is equivalent to changing $\theta_{0}$ by a constant amount and so has no effect on the final results.

\subsubsection{Two-Pan Equal-Arm Balance}

This is the oldest type of precision balance. Its basic design is represented by the "scales of justice" or by the zodiac sign for Libra.

\subsubsection{Single Substitution}

This method is very similar to single-substitution on a single-pan balance. The read-out provided by an equal-arm balance corresponds to just the screen-reading of the single-pan balance. An uncalibrated counterpoise which has nearly the same mass as $M$ and $S$ is placed on one of the pans throughout the measurement sequence. The size of the counterpoise is selected to ensure that the balance indication will be on scale with $M$ on the second pan. The weights $M, S$ and a sensitivity weight $\Delta$ are placed on the second pan in nearly the same sequence used in the one-pan case.

The required steps are as follows:

1) Place $C$ on first pan, $M$ on second pan and record indicator reading $\theta_{1}$.

2) Remove $M$

3) Place $S$ on second pan and record indicator reading $\theta_{2}$. (Recall that $S$ is a summation of calibrated weights which is chosen to be near the scale indication of M.)

4) Place the sensitivity wejght $\Delta$ on that pan which will cause the indicator to move toward the center of the scale. Record indicator reading $\theta_{3^{\circ}}$

It is convenient to choose as the second pan (i.e. the one on which $M$ and $S$ are placed) the one for which increasing increments of load give increasingly positive indicator readings.

Going through detailed arguments similar to those for single-pan, single substitution, we arrive at

$$
M^{\top}-S^{T}=\rho_{A}\left(V_{M}-V_{S}\right)+\frac{\left(\Delta^{\top}-\rho_{A} V_{\Delta}\right)}{\left|\theta_{3}-\theta_{2}\right|}\left(\theta_{1}-\theta_{2}\right)
$$

The absolute value $\left|\theta_{3}-\theta_{2}\right|$ comes about because the $\Delta$ weight might be placed on either of the two pans. Note that if the arms of the balance are not equal, the sensitivity weight must be placed on the second pan.

\subsubsection{Double-Substitution}

As in the case of the single-pan balance, one additional reading is taken for double substitution.

5) Remove $S$ from the balance; leave $\Delta$ where it is,

6) Place $M$ on the second pan and record the indicator reading $\theta_{4}$. 
By obvious extension of the preceding arguments, we arrive at the equation for double-substitution on an equal-arm balance:

$$
M^{\top}-S^{\top}=\rho_{A}\left(V_{M}-V_{S}\right)+\frac{\left(\Delta^{\top}-\rho_{A} V_{\Delta}\right)}{\left|\theta_{3}-\theta_{2}\right|} \frac{\left(\theta_{1}-\theta_{2}-\theta_{3}+\theta_{4}\right)}{2} \text {. }
$$

Again, to be strictly correct in the presence of linear drfit, the term $\theta_{3}-\theta_{2}$ can be replaced by $\left(3 \theta_{3}-3 \theta_{2}+\theta_{1}-\theta_{4}\right) / 2$. Double substitution in this case has the same advantages as weighing on a single-pan balance.

In summary, we have derived the following equations for the four types of substitution weighing we have discussed:

\begin{tabular}{lcc} 
Balance Type & Substitution Type & Equation \\
\cline { 1 - 2 } Single-Pan & Single & $(27)$ \\
Single-Pan & Double & $(30)$ \\
Two-Pan & Single & $(31)$ \\
Two-Pan & Double & $(32)$
\end{tabular}

\subsection{Transposition Weighing}

Two-pan, equal-arm balances can also be used to do transposition weighing. This method does not require a separate counterpoise and also doubles the balance sensitivity.

\subsubsection{Single Transposition}

The following steps are required:

1. Place $M$ on one of the pans, e.g., the left pan and $S$ on the right pan. $S$ should be adjusted so that the indicator reads on scale. Record the indication $\theta_{1}$.

2. Remove $M$ and $S$.

3. Replace $M$ and $S$ on the balance but in transposed position (that is, $M$ on the right pan and $S$ on the left pan). Record the reading $\theta_{2}$.

4. Add $\Delta$ to that pan which will cause the indicator to deflect towards the center of the reading scale. Record the indication $\theta_{3}$.

The three indications give the relations:

and

$$
\begin{aligned}
& M^{\top}-S^{\top} \doteq \rho_{A}\left(V_{M}-V_{S}\right)+k\left(\theta_{1}-\theta_{0}\right) \\
& S^{T}-M^{T} \doteq \rho_{A}\left(V_{S}-V_{M}\right)+k\left(\theta_{2}-\theta_{0}\right)
\end{aligned}
$$

$$
S^{T}-M^{\top} \doteq \rho_{A}\left(V_{S}-V_{M}\right) \pm\left(\Delta^{T}+\rho_{A} V_{\Delta}\right)+k\left(\theta_{3}-\theta_{0}\right) \text {. }
$$

Here again, $\theta_{0}$ is the balance indication when both pans are empty. The \pm sign is used to indicate that, in the last equation, the sign of $\left(\Delta^{\top}-\rho_{A} V_{\Delta}\right)$ depends on which pan held the sensitivity weight. 
Subtracting (34) from (35) yields

$$
k=\frac{\Delta^{\top}-\rho_{A} V_{\Delta}}{\left|\theta_{3}-\theta_{2}\right|} \text {. }
$$

where, once again, the absolute value was used to take account of whichever pan the sensitivity weight is placed on.

By subtracting (34) from (33), we have

$$
M^{\top}-S^{\top}=\rho_{A}\left(V_{M}-V_{S}\right)+\frac{k}{2}\left(\theta_{1}-\theta_{2}\right)
$$

Finally, we can substitute from (36) into (37) to obtain

$$
M^{\top}-S^{\top}=\rho_{A}\left(V_{M}-V_{S}\right)+\frac{\left(\Delta^{\top}-\rho_{A} V_{\Delta}\right)}{\left|\theta_{3}-\theta_{2}\right|} \frac{\left(\theta_{1}-\theta_{2}\right)}{2} .
$$

CAUTION: In transposition weighing, attention has to be paid to the sense of the balance scale. We have assumed above that the balance indication becomes more positive as the mass on the left pan is increased. For scales of opposite sense, the plus sign of eq. (38) should be changed to minus. Also, if the arms of the balance are insufficiently "equal," transposition weighing will result in error.

\subsubsection{Double Transposition}

The procedure for this method follows that outlined above but with the following additions:

5) Remove $M$ and $S$, leaving $\Delta$ where it is.

6) Place $M$ on the left pan and $S$ on the right pan. Record indication $\theta_{4}$.

These steps provide us with the additional equation

$$
M^{\top}-S^{\top} \doteq \rho_{A}\left(V_{M}-V_{S}\right) \pm\left(\Delta^{\top}-\rho_{A} V_{\Delta}\right)+k\left(\theta_{4}-\theta_{0}\right)
$$

where the \pm sign is needed once again since the location of the $\Delta$ weight is ambiguous.

By subtracting (35) from (39) we have

$$
M^{\top}-S^{T}=\rho_{A}\left(V_{M}-V_{S}\right)+\frac{k}{2}\left(\theta_{4}-\theta_{3}\right)
$$

and substituting for $k$ from (36) yields

$$
M^{\top}-s^{\top}=\rho_{A}\left(V_{M}-V_{S}\right)+\frac{1}{2} \frac{\left(\Delta^{\top}-\rho_{A} V_{\Delta}\right)}{\left|\theta_{3}-\theta_{2}\right|}\left(\theta_{4}-\theta_{3}\right) \text {. }
$$


Adding (40) to (38) finally results in

$$
M^{\top}-S^{\top}=\rho_{A}\left(V_{M}-V_{S}\right)+\frac{\left(\Delta^{\top}-\rho_{A} V_{\Delta}\right)}{4\left|\theta_{3}-\theta_{2}\right|}\left(\theta_{1}-\theta_{2}-\theta_{3}+\theta_{4}\right) .
$$

Again the reader is cautioned that: the plus sign in front of the second term applies only to balances whose indications become more positive as the mass in the left pan is increased; in the presence of linear drift, the term $\theta_{3}-\theta_{2}$ can be replaced by $\left(3 \theta_{3}-3 \theta_{2}+\theta_{1} \theta_{4}\right) / 2$.

\subsection{Weighing on Electronic Balances}

The most recent development in mass technology is the appearance of the completely electronic balance. This can be thought of as a single-pan balance whose screen span has become so large that dial weights are no longer necessary. These balances are rugged, easy to use, and most are directly interfaceable to computers or data loggers. Unfortunately at present they rarely attain the precision achieved by the best mechanical balances. For metrology purposes, these balances are used just as the single-pan balances discussed above and the same equations apply. If and only if experience verifies that the constant $k$ is indeed constant, can weighing procedures be simplified so that the sensitivity weight need not be measured for every substitution or double substitution.

The results we have just derived can be generalized. First, although only true mass has been dealt with, the reader should by now be able to generate the corresponding equations for apparent mass. Next, since $S$ is assumed to be an assembly of calibrated weights, all the measurements described in this section provide an estimate for $M^{\top}$, although they do not constitute an actual calibration.

Recall that we had (eq. (10))

$$
M^{\top}=S^{\top}+\rho_{A}\left(V_{M}-V_{S}\right)+k \Delta \theta
$$

or equivalently

$$
M^{\top}=\frac{s^{\top}-\rho_{A} V_{S}+k \Delta \theta}{1-\rho_{A} / \rho}
$$

Usually, the manufacturer of a weight supplies the buyer with the weight's density so that formulation (10") is more appropriate. Equation (10') is often used in computer analysis of data, however. In this case, one estimates $V_{M}$ as $V_{M} N / \rho$, where $N$ is the nominal mass value of $M$. Equation $\left(10^{\prime}\right)$ then gives a first order approximation for $M^{\top}$. This first estimate can be used to find a better value for $V_{M}$ and the process repeated until convergence is reached.

If $S$ and $M$ are both unknown and not sufficiently close in mass, known weights can be added to either $S$ or $M$. These added weights are then included in the weighing equations. 
Sometimes M itself will be a summation of weights. Let us say, to take a specific example, that $M$ is the sum of three weights whose nominal values are $50 \mathrm{mg}$, $30 \mathrm{mg}$, and $20 \mathrm{mg}$. Furthermore, the weight manufacturer specifies that the $50-\mathrm{mg}$ and $30-\mathrm{mg}$ weights are made of tantalum $\left(\rho=16.6 \mathrm{~g} / \mathrm{cm}^{3} ; \alpha=20 \times 10^{-6} /{ }^{\circ} \mathrm{C}\right)$ but the $20-\mathrm{mg}$ weight is made of aluminum $\left(\rho=2.7 \mathrm{~g} / \mathrm{cm}^{3} ; \alpha=69 \times 10^{-6} /{ }^{\circ} \mathrm{C}\right)$. What is the effective density of the summation and what is its effective coefficient of expansion?

Answer:

As a first approximation, assume the mass of each weight is equal to its nominal value. Therefore, the total volume is approximated by

$$
\frac{.050}{16.6}+\frac{0.030}{16.6}+\frac{0.020}{2.7}=0.012227 \mathrm{~cm}^{3}
$$

The effective density is then approximated by

$$
\rho \equiv M / V \approx \frac{.050+.030+.020}{0.012227}=8.18 \mathrm{~g} / \mathrm{cm}^{3} \text {. }
$$

The effective coefficient of expansion is a weighted average--the coefficients of each weight are added in proportion to the volume of the weight to the total volume:

$$
\begin{aligned}
\alpha & =\frac{20 \times 10^{-6} \times \frac{0.050}{16.6}+20 \times 10^{-6} \times \frac{0.030}{16.6}+69 \times 10^{-6} \times \frac{0.020}{2.7}}{\frac{.050}{16.6}+\frac{0.030}{16.6}+\frac{0.020}{2.7}} \\
& =50 \times 10^{-6} /{ }^{\circ} \mathrm{C}
\end{aligned}
$$

After mass values for the weights comprising $M$ have been found, the metrologist should verify that the approximations used to estimate $\rho$ and $\alpha$ were adequate. Only very rarely will this not be the case, requiring an iteration: the mass values obtained for the weights comprising $M$ are used to find new effective values of $\rho$ and $\alpha$; these latter two values are then used to calculate improved mass values. 
We will discuss two distinct programs in mass metrology: surveillance and calibration. As the name implies, surveillance attempts to monitor a calibrated assembly or set of weights. Surveillance looks for signs that one or more members of the set may have changed since the last calibration. Calibration, of course, attempts to assign the best possible value of mass to a weight by relying on a chain of traceability to universally recognized standards. Assignment of a realistic uncertainty to a calibration value is equal in importance to the calibration value itself. Several additional definitions will be useful.

\section{A standard mass $S^{\top}$ was already defined in Section 3 . We also have:}

SC: Check standard weight with true mass $(S C)^{\top}$.

This mass is basically required to monitor in-house procedures and accuracies. Usually (SC) is a calibrated standard $S$ that has been added to a set during calibration. By measuring (SC) (treated as an unknown $M$ ) versus $S$ and comparing the results with the known value one can then determine if the measuring process is either in or out of control. This type of self check should be done periodically and the values for (SC) should be monitored continuously via trend charts (see below).

Set: A group of weights in a specific order. To be of maximum utility, sets usually cover several decades of nominal mass; e.g., $1 \mathrm{mg}$ to $100 \mathrm{~g}$. Further smaller groups (called subsets) of the complete set can be selected to produce any nominal value within the span of the set in increments of the smallest set members. What this means is that, taking the example of a $100 \mathrm{~g}$ to $1 \mathrm{mg} \mathrm{set,} \mathrm{any} \mathrm{of} \mathrm{the} \mathrm{nominal} \mathrm{values}$ $0.001 \mathrm{~g}, 0.002 \mathrm{~g}, 0.003 \mathrm{~g}, \ldots, 99.998 \mathrm{~g}, 99.999 \mathrm{~g}, 100 \mathrm{~g}$ can be obtained by selecting appropriate weights from the set. Usually a set will employ the smallest number of weights possible to cover each decade. A set with a sequence 5, 3, 2, 1 could for instance range from $100 \mathrm{~g}$ to $1 \mathrm{mg}$ with the sequence repeated for each decade (e.g. $500 \mathrm{mg}, 300 \mathrm{mg}, 200 \mathrm{mg}, 100 \mathrm{mg}$ ).

Design: This specifies explicitly how and in what order a set of weights is to be compared (measured). It states in detail what weights of the set have to be used at a particular step.

ST: Transfer standard weight with true mass $(S T)^{\top}$.

As mentioned under "Set" above, in many cases a number of weights, e.g. from $1 \mathrm{~kg}$ to $10 \mathrm{~g}$ must be calibrated. These are usually divided into decade groupings; that is, weights from $1 \mathrm{~kg}$ to $100 \mathrm{~g}$ are calibrated at the same time using one design (A standard kilogram is also included in the design). Next the weights from $100 \mathrm{~g}$ to $10 \mathrm{~g}$ are calibrated--possibly using a different balance. In this procedure, it is common for the $100 \mathrm{~g}$ weight which was calibrated as a result of the first series of measurements to become the standard for the second series. The $100 \mathrm{~g}$ weight thus serves as a "transfer standard" because it is used to transfer our knowledge of a standard kilogram to weights of smaller denomination.

\subsection{Surveillance}

Two distinct types are outlined below as Type I and Type II. (For more complete detailed discussions of many different designs, the reader is referred to ref. $[4])$. 
The basic idea of surveillance testing is to ensure the self-consistency of the weight set. For example, the $20 \mathrm{~g}, 30 \mathrm{~g}$, and $50 \mathrm{~g}$ weights can be checked against the $100 \mathrm{~g}$ weight to see if the difference is within expected limits. It is also advantageous (though not essential) to compare one weight of the set (usualiy the largest) against an independent standard, S. The latter operation establishes whether the entire weight set has undergone a change--even though there may still be self-consistency within the set. The basic motivations for surveillance testing are:

to verify the values of newly calibrated weights

to establish the stability of a new weight set

to determine if an accident (such as being dropped on the floor) has damaged the weights involved.

Buoyancy corrections may not be needed in surveillance testing. One should check the magnitude of such corrections compared to the surveillance limits (see below) to see whether it is worthwhile to make the buoyancy corrections.

\subsubsection{Type I}

The object of this surveiliance method is to perform an intercomparison of all weights in a set using a minimum number of steps. It is preferable to have one standard weight as a member of the set. We will denote it as $S$ for this discussion. The standard S should always be as large as the largest member of the set; or as large as is convenient.

For the comparison measurement one always starts out with the largest weight and compares it with a summation of weights next in magnitude such that the sum is equivalent to the largest. Next, a weight from the first summation is compared with a lower summation, and the process is continued until all the weights have been used. If a standard is included in the set, then the $S$ has to be compared first with the largest mass. An example will help to visualize the procedure.

\section{Example:}

The set contains a standard weight $S$ whose nominal value is $100 \mathrm{~g}$ and weights of $100,50,30,20,10,5,3,2,1,0.5,0.3,0.2,0.1,0.05,0.03,0.02,0.01$, $0.005,0.003,0.002$ and $0.001 \mathrm{~g}$; called $M_{1}$ to $M_{21}$, respectively. Such a set is known as a set with mass ratios of $5,3,2$, 1 from $100 \mathrm{~g}$ to $1 \mathrm{mg}$. For the measurement sequence, we start out with the standard $S$ versus $M_{1}=100 \mathrm{~g}$ and work down to 1 mg such that all masses are included via a minimum number of steps. The designation $M_{i}$ refers to the nominal value of the $i$ th weight whereas $M_{j}^{\top}$ refers to the true mass value of the ith weight.

Ist Meas: $M_{1}^{\top}-S^{\top}=\delta_{1}$, where $M_{1}=100 \mathrm{~g}, S=100 \mathrm{~g}$

2nd Meas: $M_{1}^{\top}-M_{2}^{T^{\prime}}=\delta_{2}$, where $M_{2}^{\prime}=\left(M_{2}+M_{3}+M_{4}\right)$

$=(50+30+20) \mathrm{g}$

$=100 \mathrm{~g}$ 
3rd Meas: $M_{4}^{\top}-M_{3}^{T^{\prime}}=\delta_{3}$, where $M_{3}{ }^{\prime}=\left(M_{5}+M_{6}+M_{7}+M_{8}\right)$

$$
\begin{aligned}
& =(10+5+3+2) \mathrm{g} \\
& =20 \mathrm{~g}
\end{aligned}
$$

4th Meas: $M_{8}^{\top}-M_{4}^{\top}{ }^{\prime}=\delta_{4}$, where $M_{4}^{\prime}=\left(M_{9}+M_{10}+M_{11}+M_{12}\right)$

$=(1+0.5+0.3+0.2) \mathrm{g}$

$=2 \mathrm{~g}$

5th Meas: $M_{12}^{\top}-M_{5}^{T^{\prime}}=\delta_{5}$, where $M_{5}^{\prime}=\left(M_{13}+M_{14}+M_{15}+M_{16}\right)$

$=(0.1+0.05+0.03+0.02) \mathrm{g}$

$=0.2 \mathrm{~g}$

6th Meas: $M_{16}^{\top}-M_{6}^{T^{\prime}}=\delta_{6}$, where $M_{6}^{\prime}=\left(M_{17}+M_{18}+M_{19}+M_{20}\right)$

$$
\begin{aligned}
& =(0.01+0.005+0.003+0.002) \mathrm{g} \\
& =0.02 \mathrm{~g}
\end{aligned}
$$

7th Meas: $M_{19}^{\top}-M_{7}^{\top}{ }^{\prime}=\delta_{7}$, where $M_{7}^{\prime}=\left(M_{20}+M_{21}\right)$

$$
\begin{aligned}
& =(0.002+0.001) \mathrm{g} \\
& =0.003 \mathrm{~g}
\end{aligned}
$$

Note that the last measurement is amended because only $M_{21}$ is left to measure.

With a very simple software routine, the differences ( $\delta$ 's) can then be compared against the known (accepted) values. These new differences should then be plotted and compared chronologically with previous tests. Together with predetermined uncertainty limits one can then monitor the particular weight set. Usually one devotes one chart for each $\delta$. For example, fig. 5 shows a survellance chart for $\delta_{4}$. The horizontal 1 ine is the value of $\delta_{4}$ inferred from the most recent calibration report of the set. The points represent values of $\delta_{4}$ which were derived from surveillance testing. As long as the points remain within the upper and lower horizontal 7 ines, (known as the surveillance 7 imits), we have no evidence that any of the weights involved in the measurement of $\delta_{4}$ has changed from its reported calibration. If an obvious trend is apparent, however, a recalibration can be done before the surveillance limit is exceeded. Two questions remain to be answered:

(1) How are the surveillance limits determined?; and (2) If a point lies outside the surveillance limits, how does one determine which of the weights (that is $2 \mathrm{~g}, 1 \mathrm{~g}$, $500 \mathrm{mg}, 300 \mathrm{mg}$, or $200 \mathrm{mg}$ in the case of fig. 4) has changed? The answers to these questions will be deferred until after the description of Type II Surveillance.

\subsubsection{Type II}

For Type II surveillance, a more sophisticated approach to the mass comparisons is taken. Again, the procedure is best described by an example.

Suppose we consider the following three weight groupings from the set used in the example for Type I. 


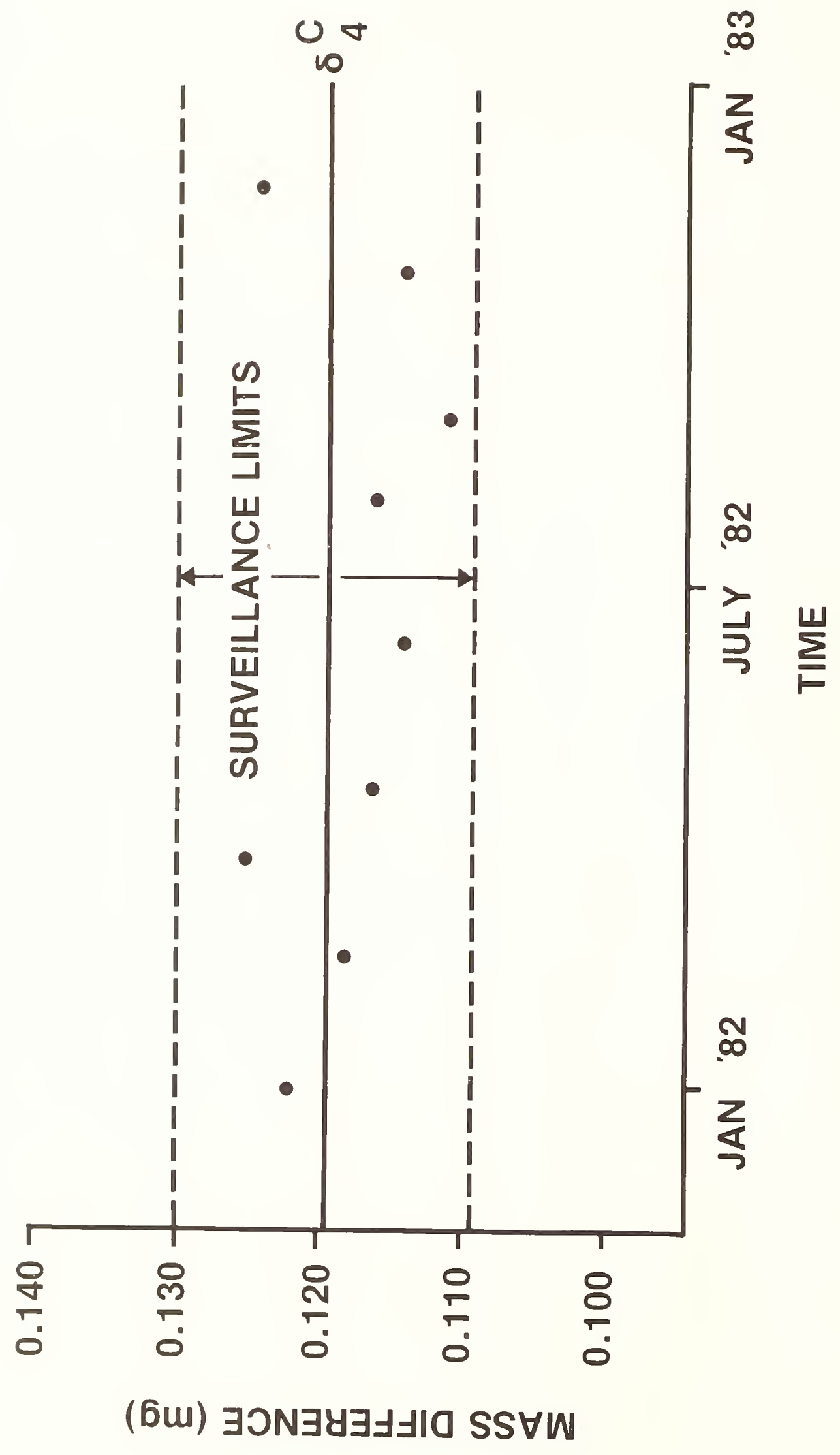

$\stackrel{0}{0}$

$4 \frac{9}{5}$

몬

क्षे

ro.

ن

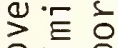

드

ज

4 纪

둥

语:

บㄹ

它引宛

ه

它递

-

$=\frac{\pi}{2}$

a.

帘苦苍苍

$\neg \backsim$

a

$\sum_{i}^{2}$

a

둰

4

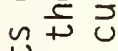

$\frac{1}{5} 4$

$\propto$ ฯ ज

난

iा

똥

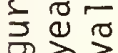

- 
Weight Grouping

$3 \mathrm{~g}$

$2 g+1 g$

$2 \mathrm{~g}+500 \mathrm{mg}+300 \mathrm{mg}+200 \mathrm{mg}$
Designation

$$
\begin{gathered}
M_{7} \\
M_{3}^{\prime \prime}=M_{8}+M_{9}=M_{8}+M_{10}+M_{11}+M_{12}
\end{gathered}
$$

For Type II surveillance, the following three mass differences are measured.

$$
\begin{aligned}
& \text { 1. } M_{7}^{T}-M_{3}^{T \prime \prime}=\delta_{1} \\
& \text { 2. } M_{7}^{T}-M_{3}^{T}{ }^{\prime \prime \prime}=\delta_{2} \\
& \text { 3. } M_{3}^{T}-M_{3}^{T \prime \prime}=\delta_{3}
\end{aligned}
$$

(Note that $\delta_{3}$ cannot be measured by transposition weighing because $M_{8}$ is common to $M_{3}$ " and $M_{3} " \prime$.)

The three weighing operations shown above form a simple "design". The concept of weighing designs is crucial to calibration operations and will be discussed at length in Section 4.2.2. Here we may simply note that we are working with a "3-1's design" (i.e., 3 different weight groupings with 1 nominal mass).

If the weighing process had no scatter (standard deviation equal to zero), then $\delta_{3}=\delta_{2}-\delta_{1}$. In that case we would derive no additional information from the $\delta_{3}$ measurement and its inclusion would, in fact, be a waste of time. of course, the process does have some scatter and the $\delta_{3}$ which we measure has only a slight probability of being equal to the measured value $\delta_{2}-\delta_{9}$. Therefore, far from being useless, the measurement of the trio $\delta_{7}, \delta_{2}, \delta_{3}$ can be used to good advantage in two ways:

First, a statistical technique known as "least squares" fitting [3,8] of the data provides better estimates of the three mass differences than can be gotten with a single measurement.

$$
\begin{aligned}
& \text { 1. } M_{7}^{\top}-M_{3}^{T^{\prime \prime}}=1 / 3\left(2 \delta_{1}+\delta_{2}-\delta_{3}\right)=\delta_{1}^{1} \\
& \text { 2. } M_{7}^{\top}-M_{3}^{T_{1 \prime \prime}}=1 / 3\left(\delta_{1}+2 \delta_{2}+\delta_{3}\right)=\delta_{2}^{1} \\
& \text { 3. } M_{3}^{\top \prime \prime}-M_{3}^{\top \prime \prime \prime}=1 / 3\left(-\delta_{1}+\delta_{2}+2 \delta_{3}\right)=\delta_{3}^{1} .
\end{aligned}
$$

The least-squares estimates $\delta_{1}^{1}, \delta_{2}^{1}$, and $\delta_{3}^{1}$ can be checked against the calibration report as in Type I surveillance (see refs. [3] and [8]).

Second, the differences between the least-squares estimates and the observations, $\left|\delta_{1}^{\prime}-\delta_{1}\right|,\left|\delta_{2}^{\prime}-\delta_{2}\right|$ and $\left|\delta_{3}^{\prime}-\delta_{3}\right|$, are related to scatter in the measurement process. ${ }^{6}$ If one of these differences is markedly higher (say three times higher)

\footnotetext{
${ }^{6}$ It is characteristic of the least squares solution to a $3-1$ 's design that $\left|\delta_{1}^{\prime}-\delta_{1}\right|$ $=\left|\delta_{2}^{1}-\delta_{2}\right|=\left|\delta_{3}^{1}-\delta_{3}\right|$. This feature cannot be generalized to other designs.
} 
than the measured standard deviation of the balance, one should redo the measurements of $\delta_{1}, \delta_{2}$, and $\delta_{3}$. If trouble persists, it is a good indication that the precision of the balance (eq. (21)) has deteriorated.

As another example we will use a set similar to the one discussed under Type I, i.e. mass ratios $5,3,2,1$ from $100 \mathrm{~g}$ to $1 \mathrm{mg}$ with $S=100 \mathrm{~g}$.

The first 3 - l's weighing series consists of

1a. $S^{\top}-M_{1}^{\top}=\delta_{1}$, where $M_{1}=100 \mathrm{~g}$

ib. $S^{\top}-M_{2}^{\top}=\delta_{2}$, where $M_{2}^{\prime}=\left(M_{2}+M_{3}+M_{4}\right)$

$=(50+30+20) \mathrm{g}$

1c. $M_{1}^{\top}-M_{2}^{T_{1}}=\delta_{3}$

$=100 \mathrm{~g}$

The next 3 - I's weighing series is

2a. $M_{3}^{T}-M_{3}^{T_{1}}=\delta_{4}$, where $M_{3}=30 \mathrm{~g}$

$$
\begin{aligned}
M_{3}^{\prime} & =\left(M_{4}+M_{5}\right) \\
& =(20+10) \mathrm{g} \\
& =30 \mathrm{~g}
\end{aligned}
$$

2b. $M_{3}^{\top}-M_{4}^{T_{1}}=\delta_{5}$, where $M_{4}^{\prime}=\left(M_{4}+M_{6}+M_{7}+M_{8}\right)$

$$
\begin{aligned}
& =(20+5+3+2) \mathrm{g} \\
& =30 \mathrm{~g}
\end{aligned}
$$

2c. $M_{3}^{T_{1}}-M_{4}^{T_{1}}=\delta_{6}$

This is followed by the third 3 - I's series as

3a. $M_{7}^{T}-M_{5}^{T}{ }^{\top}=\delta_{7}$, where $M_{7}=3 \mathrm{~g}$

$$
\begin{aligned}
M_{5}{ }^{\prime} & =\left(M_{8}+M_{9}\right) \\
& =(2+1) \mathrm{g} \\
& =3 \mathrm{~g}
\end{aligned}
$$

3b. $M_{7}^{\top}-M_{6}^{\top}{ }^{\top}=\delta_{8}$, where $M_{6}^{\prime}=\left(M_{8}+M_{10}+M_{11}+M_{12}\right)$

$$
\begin{aligned}
& =(2+0.5+0.3+0.2) \mathrm{g} \\
& =3 \mathrm{~g}
\end{aligned}
$$

3c. $M_{5}^{\top}{ }^{\prime}-M_{6}^{\top}{ }^{\prime}=\delta_{9}$ 
For the fourth sequence we have:

4a. $M_{11}^{\top}-M_{7}^{T^{\prime}}=\delta_{10}$, where $M_{11}=0.3 \mathrm{~g}$

$$
\begin{aligned}
M_{7}^{\prime} & =\left(M_{12}+M_{13}\right) \\
& =(0.2+0.1) \mathrm{g} \\
& =0.3 \mathrm{~g}
\end{aligned}
$$

4b. $M_{11}^{\top}-M_{8}^{\top}{ }^{\top}=\delta_{11}$, where $M_{8}{ }^{\prime}=\left(M_{12}+M_{14}+M_{15}+M_{16}\right)$

$$
\begin{aligned}
& =(0.2+0.05+0.03+0.02) \mathrm{g} \\
& =0.3 \mathrm{~g}
\end{aligned}
$$

4c. $M_{7}^{T^{\prime}}-M_{8}^{T^{\prime}}=\delta_{12}$.

For the fifth series we measure:

5a. $M_{15}^{\top}-M_{9}^{T_{1}}=\delta_{13}$, where $M_{15}=0.03 \mathrm{~g}$

$$
\begin{aligned}
M_{9}^{\prime} & =\left(M_{16}+M_{17}\right) \\
& =(0.02+0.01) \mathrm{g} \\
& =0.03 \mathrm{~g}
\end{aligned}
$$

5b. $M_{15}^{\top}-M_{10}^{\top}=\delta_{14}$, where $M_{10}^{\prime}=\left(M_{16}+M_{18}+M_{19}+M_{20}\right)$

$$
\begin{aligned}
& =(0.02+0.005+0.003+0.002) \mathrm{g} \\
& =0.03 \mathrm{~g}
\end{aligned}
$$

5c. $M_{9}^{\top}{ }^{\prime}-M_{10}^{\top}{ }^{\prime}=\delta_{15^{\circ}}$.

Finally, we arrive at the last series with

6a. $M_{19}^{\top}-M_{11}^{\top}=\delta_{16}$ where $M_{19}=0.003 \mathrm{~g}$

$$
\begin{aligned}
M_{11}{ }^{\prime} & =\left(M_{20}+M_{21}\right) \\
= & (0.002+0.001) \mathrm{g}=0.003 \mathrm{~g}
\end{aligned}
$$

6b. $M_{19}^{\top}-M_{12}^{\top}{ }^{\prime}=\delta_{17}$ where $M_{12}{ }^{\prime}=\left(M_{20}+M_{22}^{*}\right)$

$$
=(0.002+0.001 *) \mathrm{g}=0.003 \mathrm{~g}
$$

6c. $M_{11}^{\top}-M_{12}^{\top}=\delta_{18}$. 
Once again the least squares fitting technique provides better estimates such that eqs. (1a), (1b), and (1c) yield

$$
\begin{aligned}
& \left.1 a^{\prime}\right) \quad 1 / 3\left(2 \delta_{1}+\delta_{2}-\delta_{3}\right)=\delta_{1}^{\prime} \\
& \left.2 b^{\prime}\right) \quad 1 / 3\left(\delta_{1}+2 \delta_{2}+\delta_{3}\right)=\delta_{2}^{\prime} \\
& \left.3 c^{\prime}\right) \quad 1 / 3\left(-\delta_{1}+\delta_{2}+2 \delta_{3}\right)=\delta_{3}^{\prime} .
\end{aligned}
$$

If for any reason a suitable standard, $S$, is unavailable, then any uncalibrated weight or summation of weights of the correct nominal value can be used in eqs. (1a) and (1b). However, in this case only $\delta_{3}^{1}$ can be checked against the reported (known) calibration and only internal consistency of the entire set can be determined.

We also have

$\left.2 a^{\prime}\right) \quad 1 / 3\left(2 \delta_{4}+\delta_{5}-\delta_{6}\right)=\delta_{4}^{\prime}$

$\left.2 b^{\prime}\right) \quad 1 / 3\left(\delta_{4}+2 \delta_{5}+\delta_{6}\right)=\delta_{5}^{\prime}$

and

$\left.2 c^{\prime}\right) \quad 1 / 3\left(-\delta_{4}+\delta_{5}+2 \delta_{6}\right)=\delta_{6}^{\prime}$

$\left.3 a^{\prime}\right) \quad 1 / 3\left(2 \delta_{7}+\delta_{8}-\delta_{g}\right)=\delta_{7}^{\prime}$

$\left.3 b^{\prime}\right) 1 / 3\left(\delta_{7}+2 \delta_{8}+\delta_{9}\right)=\delta_{8}^{\prime}$

$\left.3 c^{\prime}\right) 1 / 3\left(-\delta_{7}+\delta_{8}+2 \delta_{9}\right)=\delta_{9}^{\prime}$

as well as

$$
\begin{aligned}
& \left.4 a^{\prime}\right) \quad 1 / 3\left(2 \delta_{10}+\delta_{11}-\delta_{12}\right)=\delta_{10}^{\prime} \\
& \left.4 b^{\prime}\right) \quad 1 / 3\left(\delta_{10}+2 \delta_{11}+\delta_{12}\right)=\delta_{11}^{\prime} \\
& \left.4 c^{\prime}\right) \quad 1 / 3\left(-\delta_{10}+\delta_{11}+2 \delta_{12}\right)=\delta_{12}^{\prime}
\end{aligned}
$$

Finally

$$
\begin{aligned}
& \left.5 a^{\prime}\right) \quad 1 / 3\left(2 \delta_{13}+\delta_{14}-\delta_{15}\right)=\delta_{13}^{\prime} \\
& \left.5 b^{\prime}\right) \quad 1 / 3\left(\delta_{13}+2 \delta_{14}+\delta_{15}\right)=\delta_{14}^{\prime} \\
& \left.5 c^{\prime}\right) \quad 1 / 3\left(-\delta_{13}+\delta_{14}+2 \delta_{15}\right)=\delta_{15}^{\prime}
\end{aligned}
$$

and

$$
\begin{aligned}
& \left.6 a^{\prime}\right) \quad 1 / 3\left(2 \delta_{16}+\delta_{17}-\delta_{18}\right)=\delta_{16}^{\prime} \\
& \left.6 b^{\prime}\right) \quad 1 / 3\left(\delta_{16}+2 \delta_{17}+\delta_{18}\right)=\delta_{17}^{\prime} \\
& \left.6 c^{\prime}\right) \quad 1 / 3\left(-\delta_{16}+\delta_{17}+2 \delta_{18}\right)=\delta_{18}^{\prime}
\end{aligned}
$$

The differences obtained for the first two results of each 3-1's series (i.e. a and b) should be compared to accepted values derived from the most recent calibration report. 
The above examples of surveillance measurements for Type I and Type II present a simplified picture of the equations. All $\delta^{\prime} s$ must contain any necessary buoyancy corrections for true mass differences. Different $\delta$ 's are obtained if apparent masses are used. From the simplified eq. (19a), one finds

$$
M^{A}-S^{A}=k \Delta \theta+\left(\rho_{A}-\rho_{0}\right)\left(V_{O M}-V_{O S}\right)=\delta
$$

where we assumed that the mass of the standard is given in apparent mass units, $S^{A}$.

Deviations of the measurements from values inferred from the most recent calibration report are evident from the previously mentioned surveitlance charts.

\subsubsection{Surveillance Limits}

Upon carrying out a surveillance test of either Type I or II it will become evident that, after any necessary buoyancy corrections have been made, the measured values $\delta_{i}$ (Type I) or the predicted values $\delta_{j}^{\prime}$ (Type II) do not exactly agree with those values, $\delta_{j}^{c}$, calculated from the calibration certificate accompanying the set. To judge how serious the disagreement is, one must also calculate surveillance limits. The surveillance limits associated with each $\delta_{i}$ or $\delta_{i}^{\prime}$ define the limits of credibility that the difference $\delta_{i}^{c}-\delta_{i}$ or $\delta_{i}^{c}-\delta_{i}^{c}$ could be due to a combination of calibration uncertainties of the weight set and the random error of the measurement which is associated with the balance used in the surveillance measurements.

Let us designate to random and systematic error limits by SL;

$$
(S L)=U+E
$$

where

$$
U=\text { systematic error as determined from the calibration report }
$$

and

$$
\begin{aligned}
& E=1 \text { imit to random error }=3 \sigma \\
& \sigma=\text { standard deviation of the measurement (refer to Appendix } D \text { for }
\end{aligned}
$$
information on estimating this number).

Then any value $\delta_{j}$ or $\delta_{i}^{\prime}$ should fall between $\delta_{j}^{c}+S L$, as shown in fig. 5 . The value of $3 \sigma$ gives a 99.7 percent confidence level for the random error (i.e. if the measurements were repeated a great many times there is a 99.7 percent chance the average would be within $\pm E$ of the result of a single surveiliance test).

For surveillance, $U$ is the root-sum-square of the individually reported calibration uncertainties such that

$$
u=\left(\Sigma u_{i}^{2}\right)^{1 / 2}
$$

Combining the uncertainties in this way is strictly valid only if they are uncorrelated. It is the nature of calibration designs, however, that the uncertainties of weights within the set generally are correlated. Thus one should view eq. (43) as an approximation which is adequate for surveillance limits. The reader is referred to the discussion of calibration uncertainties below for a fuller explanation.

The standard deviation used is known from many previous measurements (eq. (21)). It is a measure of the random errors in the balance being used.

At this stage it is worthwhile to quote a couple of examples and step through the process. From Type I surveiliance, we have 


$$
\begin{aligned}
& S=10 \mathrm{~g} \\
& M_{1}=100 \mathrm{~g} \\
& M_{2}^{\prime}=100 \mathrm{~g}=50 \mathrm{~g}+30 \mathrm{~g}+20 \mathrm{~g}=M_{2}+M_{3}+M_{4} .
\end{aligned}
$$

Assume the following previously reported calibration:

$$
\begin{array}{ll}
S^{\top}=100.0010196 \mathrm{~g} & \text { Uncertainty } \\
M_{1}^{\top}=100.0009407 \mathrm{~g} & U_{S}=0.015 \mathrm{mg} \\
M_{2}^{\top}=50.0004628 \mathrm{~g} & U_{M_{1}}=0.020 \mathrm{mg} \\
M_{3}^{\top}=30.0002926 \mathrm{~g} & U_{M_{2}}=0.011 \mathrm{mg} \\
M_{4}^{\top}=20.0001578 \mathrm{~g} & U_{M_{3}}=0.012 \mathrm{mg}
\end{array}
$$

Also assume that $\sigma=0.026 \mathrm{mg}$.

Since the first measurement is

$$
M_{1}^{\top}-S^{\top}=\delta_{1} ; \delta_{1}^{c}=-0.0000789 \mathrm{~g}=-0.079 \mathrm{mg}
$$

and we have

$$
\begin{aligned}
U & =\sqrt{U_{S}^{2}+U_{M_{1}}^{2}} \\
& =\sqrt{(0.015)^{2}+(0.020)^{2}}=0.025 \mathrm{mg}
\end{aligned}
$$

and

$$
\begin{aligned}
(S L) & =U+3 \sigma \\
& =0.025+3 \times 0.026 \\
& =0.103 \mathrm{mg} .
\end{aligned}
$$

Hence $\left(M_{1}^{\top}-S^{\top}\right)$ values should fall in the range of $(-0.079 \pm 0.103) \mathrm{mg}$.

For the second measurement we have

so that

$$
M_{1}^{\top}-M_{2}^{\top}=\delta_{2} ; \delta_{2}^{c}=0.028 \mathrm{mg}
$$

$$
\begin{aligned}
U_{M_{2}} & =\sqrt{U_{M_{2}}^{2}+U_{M_{3}}^{2}+U_{M_{4}}^{2}} \\
& =0.019 \mathrm{mg} \\
U & =\sqrt{U_{M_{2}^{\prime}}^{2}+U_{M_{1}}^{2}} \\
& =\sqrt{(0.019)^{2}+(0.020)^{2}} \\
& =0.028 \mathrm{mg}
\end{aligned}
$$




$$
=0.106 \mathrm{mg}
$$

and $M_{1}^{\top}-M_{2}^{T_{1}}$ should fall in the range

$$
0.028 \pm 0.106 \mathrm{mg} \text {. }
$$

Next, we consider the example from Type II. The first series of the design consists of $S, M_{7}$, and $M_{2}^{\prime}$ with $M_{2}^{\prime}=M_{2}+M_{3}+M_{4}$. Let us assume the same calibration report as above, with the scale standard deviation at $\sigma=0.026 \mathrm{mg}$. In this case we have three measurements

$$
\begin{aligned}
& s^{\top}-M_{1}^{\top}=\delta_{1} \\
& s^{\top}-M_{2}^{T_{1}}=\delta_{2} \\
& M_{1}^{\top}-M_{2}^{T_{1}}=\delta_{3}
\end{aligned}
$$

Because we have used a 3 - l's design, the standard deviation of our result is not simply the standard deviation of a single measurement, $\sigma$, but is instead $\sqrt{2 / 3} \sigma$. A proof of this conclusion is beyond the scope of this text but is explained fully in ref. [3].

There are several surveillance limits that can be calculated. For the three differences, we follow the calculations in Type I (but we have to remember that we are in Type II so that the standard deviation is $\sqrt{2 / 3} \sigma$ ).

Limits for $S^{\top}-M_{1}^{\top}$

We then have

$$
U=\sqrt{U_{S}^{2}+U_{M_{1}}^{2}}=\sqrt{(0.015)^{2}+(0.020)^{2}}=0.025 \mathrm{mg}
$$

$$
\begin{aligned}
(S L)=U+3 \times(\sqrt{2 / 3} \sigma) & =0.025+3 \sqrt{2 / 3} \times 0.026 \\
& =0.089 \mathrm{mg}
\end{aligned}
$$

so that

$$
\left(S^{\top}-M_{1}^{\top}\right)=(0.079 \pm 0.089) \mathrm{mg}
$$

Limits for $S^{\top}-M_{2}^{T_{1}}$

$$
U=\sqrt{U_{S}^{2}+U_{M_{2}{ }^{\prime 2}}}
$$

where

$$
U_{M_{2}^{\prime}}=\sqrt{U_{M_{2}}^{2}+U_{M_{3}}^{2}+U_{M_{4}}^{2}}=\sqrt{(0.011)^{2}+(0.012)^{2}+(0.010)^{2}}
$$

so that

$$
=0.019 \mathrm{mg}
$$

Hence

$$
U=\sqrt{(0.015)^{2}+(0.019)^{2}}=0.024 \mathrm{mg} .
$$

and

$$
(S L)=U+3 \sqrt{2 / 3} \sigma=0.024+3 \sqrt{2 / 3} 0.026=0.088 \mathrm{mg}
$$

$$
\left(S^{\top}-M_{2}^{\top}{ }^{\prime}\right)=(0.106 \pm 0.088) \mathrm{mg} \text {. }
$$


If a measurement falls outside the surveillance limits, it becomes necessary to determine which individual weights are responsible. Simple deductive reasoning is all that is required, although a few extra weighing combinations may also be needed if Type I surveillance was used.

As an example, suppose that in the Type I measurements shown earlier (4.1.1) the value for $\delta_{4}$ was outside but the value for $\delta_{3}$ was inside the surveillance limits.

Al1 we know at this point is that there is some change in the subset $M_{4}^{1}=1 \mathrm{~g}+0.5 \mathrm{~g}$ $+0.3 \mathrm{~g}+0.2 \mathrm{~g}$. Three additional weighings are now required to pinpoint the cause of the discrepancy. Recal1 that $M_{9}=1 \mathrm{~g}, M_{10}=0.5 \mathrm{~g}, M_{11}=0.3 \mathrm{~g}, M_{12}=0.2 \mathrm{~g}$, $M_{13}=0.1 \mathrm{~g}$.

We then measure

$$
\begin{aligned}
& \left.4^{\prime}\right) M_{9}^{\top}-M_{4}^{\top \prime}=\delta_{4}^{\prime \prime} \text {, where } M_{4}^{\prime \prime}=M_{10}+M_{11}+M_{12} \\
& \left.4^{\prime \prime}\right) M_{10}^{\top}-M_{5}^{\top \prime \prime}=\delta_{5}^{\prime \prime} \text {, where } M_{5}^{\prime \prime}=M_{11}+M_{12} \\
& \left.4^{\prime \prime \prime}\right) M_{11}^{\top}-M_{6}^{\top \prime \prime}=\delta_{6}^{\prime \prime} \text {, where } M_{6}^{\prime \prime}=M_{12}+M_{13}
\end{aligned}
$$

After the surveillance limits for $\delta_{4}^{\prime \prime}, \delta_{5}^{\prime \prime}, \delta_{6}^{\prime \prime}$ are calculated one investigates the results:

1.) If $\delta_{4}^{\prime \prime}$ lies outside the surveillance limits but $\delta_{5}^{\prime \prime}$ and $\delta_{6}^{\prime \prime}$ do not, then it is probable that $M_{g}$ has changed since its last calibration.

2.) If $\delta_{4}^{\prime \prime}$ and $\delta_{5}^{\prime \prime}$ lie outside the surveillance limits by opposite amounts and $\delta_{6}^{\prime \prime}$ is inside, then it is probable that the $M_{10}(0.5 \mathrm{~g})$ weight has changed.

3.) If $\delta_{4}^{\prime \prime}$ and $\delta_{5}^{\prime \prime}$ lie outside the limits by about the same amount and $\delta_{6}^{\prime \prime}$ is outside also by the same amount but in the opposite direction, then it is probable that the $M_{11}(0.3 \mathrm{~g})$ weight has changed.

4.) If $\delta_{4}^{\prime \prime}, \delta_{5}^{\prime \prime}$, and $\delta_{6}^{\prime \prime}$ all lie outside the limits by about the same amount in the same direction, then it is probable the $M_{12}(0.2 \mathrm{~g})$ has changed.

If none of the above conditions are met, then it is probable that more than one weight has changed. The reader should then consult ref. [4] for a more thorough analys is of surveillance methods.

\subsection{Calibration}

The process of "calibration" assigns mass values to weights by comparing the unknown weights to recognized standards. An uncertainty limit--3 times the standard deviation of the measurement process, plus estimated uncertainties systematic to the measurement process--accompanies each calibrated value. To an even greater degree than in Type II surveillance, redundant information is gathered in order to determine whether the measurement scatter is acceptable. A powerful self-consistency check of the calibration process is also included. 
Most of the following information can be found in much greater detail in refs. [1] and [3]. It is repeated here mainly for explaining the overall approach of the program.

Weight sets come in various denominations. The following groupings are frequently calibrated:

1. Nominally Equal Groups

These sets consist of weights, all of which have the same nominal value. The number of weights can go from 3 to as high as 13 or more. (Sets of up to 50 members have been utilized.) For large sets, subsets are usually analyzed.

Otherwise the number of weighing operations would become impractically large.

2. Groups for 2, 2,... 1, 1. . sets.

Several combinations are in use such as 2, 1, 1, 1, or 2, 2, 1, 1, 1, or 2, 1, 1,1 , or $2,2,1,1,1$, etc. Here 2 and 1 imply the same decade of mass such as $2 \mathrm{~kg}$ and $1 \mathrm{~kg}$.

3. Binary and Miscellaneous Groups

These sets are usually comprised of pound units. Many combinations are available, the use of which is slowly diminishing. A few examples provided in ref. [3] are $4,3,2,1,1$, or $10,5,2,2,1,1$, or $6,5,4,3,2,1$, etc. Note that a combination of the lower masses always sums to the value of a higher mass unit.

4. The 5, 3, 2, 1 and 5, 2, 2, 1 Groups

These are the sets most widely used. In general a set of weights in this group can span many decades with each decade comprising, for example, the 5, 3, 2, 1 sequence. In many cases standards or "check standards" (see below) are added to the sets. Examples of such groups could be $5,5,3,2,1,1$, or 5, 3, 2, 1, 1 , etc.

4.2.1. Trend Elimination in Direct Reading Balances

If a single-pan, direct reading balance has truly constant sensitivity and is subject to only a slight linear drift, the following simplification is possible:

Suppose there are four nominaliy equal weights, designated $A, B, C$, and $D$ to be compared in a particular calibration scheme, the following eight "direct" weighings are done:

(1) Place $A$ on balance and read $\theta_{1}$

(2) Remove $A$

(3) Place $B$ on balance and read $\theta_{2}$

(4) Remove B

(5) Place $C$ on balance and read $\theta_{3}$

(6) Remove C

(7) Place $D$ on balance and read $\theta_{4}$

(8) Remove $D$

(9) Replace $D$ on balance and read $\theta_{5}$

(10) Remove D

(11) Place $C$ on balance and read $\theta_{6}$

(12) Remove $C$

(13) Place $B$ on balance and read $\theta_{7}$ 
(14) Remove B

(15) Place $A$ on balance and read $\theta_{8}$

We have taken the weights in the following order: $A, B, C, D, D, C, B, A$. We then make the following calculations:

$$
\begin{aligned}
& \theta_{A}=1 / 2\left(\theta_{1}+\theta_{8}\right) \\
& \theta_{B}=1 / 2\left(\theta_{2}+\theta_{7}\right) \\
& \theta_{C}=1 / 2\left(\theta_{3}+\theta_{6}\right) \\
& \theta_{D}=1 / 2\left(\theta_{4}+\theta_{5}\right)
\end{aligned}
$$

Then the estimated difference in mass between any two weights (say $C$ and $D$ ) is

$$
C^{\top}-D^{\top}=\rho_{A}\left(V_{C}-V_{D}\right)+k\left(\theta_{C}-\theta_{D}\right)
$$

where we assume $k$ is well-known and constant. The symmetry of the weighing sequence removes problems caused by any linear drifts in the balance or weighing conditions.

The above example is an instance of "trend elimination." (Double substitution is another instance.) When weighings are subject to large air buoyancy corrections, drifts in temperature or barometric pressure may lead to errors if those quantities are only read once during all the measurements required by the design. Some calibration schemes, however, have the property of "trend elimination" even for this problem [3].

\subsubsection{Designs}

Sets or subsets of weights are calibrated together by means of a weighing design. A design simply prescribes what weighings are to be made. Each weighing is used to estimate a mass difference between two nominally equal weights or groups of weights in the set.

A typical design for a group, say $5,3,2,1,1,1$, could be as follows:

\section{Mass}

Observation

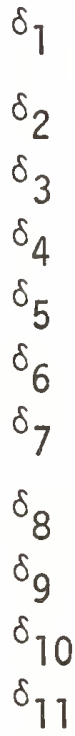

$\begin{array}{llllll}M_{5} & M_{3} & M_{2} & M_{1} & M_{\Sigma 1} & S C\end{array}$

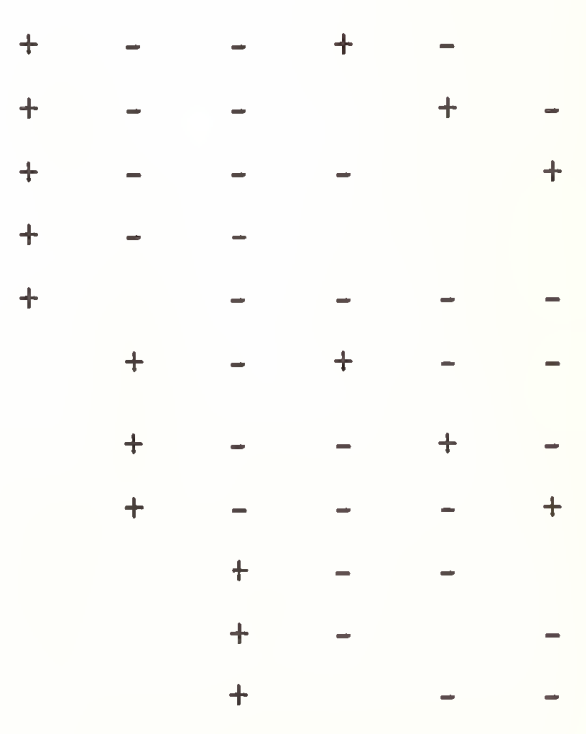


Here $+/$ - signs indicate the weight starts out on the left/right pan, for transposition weighing, or that the weight is the first/second in substitution weighing. We notice that in this group there are six masses, which must be determined $(K=6), 11$ measurements $(N=11)$, and suppose 1 restraint (e.g. the mass of the $5+3+2$ summation is known from previous measurements, $L=1$ ). We can then calculate the number of degrees of freedom (D.F.) for this design as

$$
\text { D.F. }=N-K+L=11-6+1=\underline{6} \text {. }
$$

From the 11 observations and the given value of the restraint one can then use the least squares method to solve all equations to obtain best values of all differences and therefore all masses, standard deviations, and variances. Such fitting techniques are well described in Iiterature available from the NBS [3]. (See Appendix D.)

In general the more degrees of freedom provided by a particular design, the lower the estimates of stancard deviation. Recall the previous section on surveillance testing. Each measurement in Type I surveillance had no degrees of freedom. That is, we needed to determine a quantity $\delta_{j}$ and we had only one measurement of that quantity $(K=1, N=1, L=0 ; D . F=0)$. In Type II surveillance, however, we used a simple $3-1$ design: Three quantities were determined, for example $\delta_{1}^{1}, \delta_{2}^{1}, \delta_{3}^{1}$ $(N=3)$, three measurements were made $\left(\delta_{1}, \delta_{2}, \delta_{3}\right.$, so that $\left.K=3\right)$, and there was one restraint $\left(\delta_{3}^{1}=\delta_{2}^{1}-\delta_{1}^{1}\right)$. Thus D.F. $=3-3+1=1$. The appearance of a degree of freedom allowed a crude statistical check (e.g., whether $\left|\delta_{1}-\delta_{1}^{1}\right|<$ balance standard deviation) and also led to a slightly reduced standard deviation $(\sqrt{2 / 3} \sigma$ instead of б).

The design illustrated in this section is much more sophisticated than the 3-1 but is motivated by the same twin desires for good statistical analysis and small standard deviation of the calibrated masses. The design in the above example is shown in figure 6 , where it is designated C.2. This figure is reproduced from ref. [3]. Note that two different restraints are considered: restraint $A$ is that the mass of the summation of the 5,3 , and 2 weights is known; ${ }^{7}$ restraint $B$ is that the mass of a single "1" weight is the known standard. 8 We will discuss the results based on restraint $A$ in detail. That is, we will assume that the true mass of $M_{5}+M_{3}+M_{2}$ is known. Let us designate this mass as $R^{\top}$.

In the 532111 design shown above, we have labeled one of the weights as $\Sigma 1$. This indicates that we could calibrate the $5+3+2$ summation of the next lower decade of the same weight set which could then serve as a transfer standard for a subsequent design. By "SC" we designate a weight which is external to the set being calibrated and which will serve as check standard.

${ }^{7}$ This would be useful, for instance, if the $5+3+2$ summation had been calibrated in a previous design. In this case, the $5+3+2$ summation would be a transfer standard. This restraint is used in working down in mass from $1 \mathrm{~kg}$.

${ }^{8}$ This would be useful in working up in mass from $1 \mathrm{~kg}$. 
The least-squares solutions for the five unknown weight are [3]:

$$
\begin{aligned}
& \hat{M}_{5}^{\top}=(1 / 920)\left\{100\left(\delta_{7}+\delta_{2}+\delta_{3}+\delta_{4}\right)+60 \delta_{5}-20\left(\delta_{6}+\delta_{7}+\delta_{8}+\delta_{9}+\delta_{10}+\delta_{11}\right)+460 R^{\top}\right\} \\
& \hat{M}_{3}^{\top}=(1 / 920)\left\{-68\left(\delta_{7}+\delta_{2}+\delta_{3}+\delta_{4}\right)-4 \delta_{5}+124\left(\delta_{6}+\delta_{7}+\delta_{8}\right)-60\left(\delta_{9}+\delta_{10}+\delta_{17}\right)+276 R^{\top}\right\} \\
& \hat{M}_{2}^{\top}=(1 / 920)\left\{-32\left(\delta_{7}+\delta_{2}+\delta_{3}+\delta_{4}\right)-56 \delta_{5}-104\left(\delta_{6}+\delta_{7}+\delta_{8}\right)+80\left(\delta_{9}+\delta_{10}+\delta_{11}\right)+184 R^{\top}\right\} \\
& \hat{M}_{1}^{\top}=(1 / 920)\left\{119 \delta_{7}+4 \delta_{2}-111 \delta_{3}+4 \delta_{4}-108 \delta_{5}+128 \delta_{6}-102\left(\delta_{7}+\delta_{8}\right)-125\left(\delta_{9}+\delta_{10}\right)-10 \delta_{11}+92 R^{\top}\right\} \\
& \hat{M}_{\Sigma]}^{\top}=(1 / 920)\left\{-111 \delta_{1}+119 \delta_{2}+4\left(\delta_{3}+\delta_{4}\right)-108 \delta_{5}-102 \delta_{6}+128 \delta_{7}-102 \delta_{8}-125 \delta_{9}-10 \delta_{10}-125 \delta_{11}+92 R^{\top}\right\}
\end{aligned}
$$

In addition, the least-squares solution provides a value for the check standard:

$$
(\hat{S C})^{\top}=(1 / 920)\left\{4 \delta_{7}-111 \delta_{2}+119 \delta_{3}+4 \delta_{4}-108 \delta_{5}-102\left(\delta_{6}+\delta_{7}\right)+128 \delta_{8}-10 \delta_{9}-125\left(\delta_{10}+\delta_{11}\right)+92 R^{\top}\right\}
$$

The metrologist must determine the values $\delta_{1}, \delta_{2}, \ldots \delta_{M}$ and the value of $R^{\top}$ by experiment. The least-squares solution shows how to combine the observations with the value of the restraint in order to arrive at mass values for the weights used in the design.

In addition to mass values, the least-squares solution provides fitted values for the observations, which when compared with the measured values give:

$$
\begin{aligned}
& \delta_{1}-\delta_{1}^{\prime}=\delta_{1}-\hat{M}_{5}^{\top}+\hat{M}_{3}^{\top}+\hat{M}_{2}^{\top}-\hat{M}_{1}^{\top}+\hat{M}_{\Sigma 1}^{\top} \\
& \delta_{2}-\delta_{2}^{\prime}=\delta_{2}-\hat{M}_{5}^{\top}+\hat{M}_{3}^{\top}+\hat{M}_{2}^{\top}-\hat{M}_{\Sigma 1}^{\prime} T^{\top}+(\hat{S C})^{\top} \\
& \delta_{3}-\delta_{3}^{\prime}=\delta_{3}-\hat{M}_{5}^{\top}+\hat{M}_{3}^{\top}+\hat{M}_{2}^{\top}+\hat{M}_{7}^{\top}-(\hat{S C})^{\top} \\
& \delta_{4}-\delta_{4}^{\prime}=\delta_{4}-\hat{M}_{5}^{\top}+\hat{M}_{3}^{\top}+\hat{M}_{2}^{\top} \\
& \delta_{5}-\delta_{5}^{\prime}=\delta_{5}-\hat{M}_{5}^{\top}+\hat{M}_{2}^{\top}+\hat{M}_{7}^{\top}+\hat{M}_{\Sigma 1}^{\top}+(\hat{S C})^{\top} \\
& \delta_{6}-\delta_{6}^{\prime}=\delta_{6}-\hat{M}_{3}^{\top}+\hat{M}_{2}^{\top}-\hat{M}_{7}^{\top}+\hat{M}_{\Sigma 1}^{\top}+(\hat{S C})^{\top} \\
& \delta_{7}-\delta_{7}^{1}=\delta_{7}-\hat{M}_{3}^{\top}+\hat{M}_{2}^{\top}+\hat{M}_{7}^{\top}-\hat{M}_{\Sigma 7}^{\top}+(\hat{S C})^{\top} \\
& \delta_{8}-\delta_{8}^{\prime}=\delta_{8}-\hat{M}_{3}^{\top}+\hat{M}_{2}^{\top}+\hat{M}_{7}^{\top}+\hat{M}_{\Sigma 7}^{\top}-(\hat{S C})^{\top} \\
& \delta_{9}-\delta_{9}^{\prime}=\delta_{9}-\hat{M}_{2}^{\top}+\hat{M}_{1}^{\top}+\hat{M}_{\Sigma 1}^{\top} \\
& \delta_{10}-\delta_{10}^{1}=\delta_{10}-\hat{M}_{2}^{\top}+\hat{M}_{1}^{\top}+(\hat{S C})^{\top} \\
& \delta_{11}-\delta_{11}^{\prime}=\delta_{11}-\hat{M}_{2}^{\top}+\hat{M}_{\Sigma 1}^{\top}+(\hat{S C})^{\top}
\end{aligned}
$$


These deviations are useful for two reasons. First, the estimated standard deviation of the least-squares fit is equal to

$$
\frac{1}{N-K+1}\left(\sum_{i=1}^{N}\left(\delta_{i}-\delta_{i}^{\prime}\right)^{2}\right)^{1 / 2} .
$$

Second, a glance at each of the eleven values of $\delta_{i}-\delta_{i}^{1}$ can often pinpoint the source of a blunder in entering the raw data into a computer--that is, if a blunder has been made, the value of $\delta_{j}-\delta_{j}^{\prime}$ affected by the mistake will often appear much larger than all the other values.

Finally, the least-squares solution also provides estimates of the standard deviation for each computed mass and each combination of mass. Below the design in fig. 6 is a table called "Factors for Computing Standard Deviations." Suppose the standard deviation of the measurement process is $\sigma$. Then if design $C .2$ is used to calibrate the mass of the "5" weight, for example, the table tells us that the standard deviation assigned to this mass, $\sigma_{5}$, will be:

\section{$0.2331 \times \sigma$ if restraint $A$ were used \\ $1.7846 \times \sigma$ if restraint $B$ were used.}

Also from the table we see that the standard deviation of the sum of the 5 and 3 weights, $\sigma_{8}$, is given by:

\section{$0.2638 \times \sigma$ if restraint $A$ were used \\ $2.8284 \times \sigma$ if restraint $B$ were used.}

It is typical of least squares results that, for example,

In this case,

$$
\sigma_{m+n} \neq\left(\sigma_{m}^{2}+\sigma_{n}^{2}\right)^{1 / 2} \text {. }
$$

$$
\begin{aligned}
& \sigma_{8}<\left(\sigma_{5}{ }^{2}+\sigma_{3}{ }^{2}\right)^{1 / 2} \text { for restraint } A \\
& \sigma_{8}>\left(\sigma_{5}{ }^{2}+\sigma_{3}{ }^{2}\right)^{1 / 2} \text { for restraint } B .
\end{aligned}
$$

Thus the metrologist must choose both design and restraint carefully to minimize the resulting standard deviations.

\subsubsection{Statistical Checks}

Computer programs, such as those developed by NBS, are routinely utilized in mass calibration laboratories. The user supplies all the measured data for the set, all environmental conditions, and other necessary data. The program then provides:

1. a detailed listing of data provided

2. the least squares fit, i.e. the desired mass values

3 . the "F ratio" and the "t value".

Two crucial assumptions underlie the calibration of unknown weights by leastsquares fitting of design data: 
OESIGN C.I

$5 \cdot 3 \cdot 2 \cdot 1 \cdot 1$

$K=5$

(HA TFDRO)

oEsefVAT IONS

$r(1)$
$Y(2)$
$Y(3)$
$Y(4)$
$Y(5)$
$r(6)$
$Y(7)$
$r(A)$

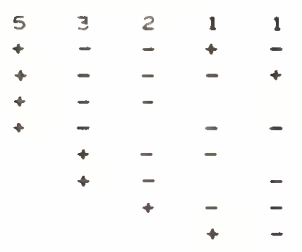

RESTRAINT A

RESTRAINT B

FACTORS FOR COMPUTING ST DEVS

$$
\begin{array}{r}
1 \\
1 \\
2 \\
3 \\
4 \\
5 \\
6 \\
7 \\
8 \\
5 \\
10
\end{array}
$$

\begin{tabular}{|c|c|c|c|c|c|}
\hline \multicolumn{2}{|c|}{ RESTRAINTS } & \multirow[b]{2}{*}{5} & \multirow[b]{2}{*}{3} & \multirow[b]{2}{*}{2} & \multirow[b]{2}{*}{1} \\
\hline A & 8 & & & & \\
\hline .4175 & .0000 & & & & \\
\hline .4175 & .5345 & & & & + \\
\hline .3546 & .9258 & & & + & \\
\hline .3338 & 1.3093 & & + & & \\
\hline .5237 & 1.6903 & & + & & + \\
\hline .2673 & 2.0702 & + & & & \\
\hline .5237 & 2.4495 & + & & & + \\
\hline .2338 & 2.9277 & + & & + & \\
\hline .3546 & 3.3381 & + & + & & \\
\hline .5632 & 3.7033 & + & + & & + \\
\hline .0000 & 4.1748 & + & + & + & \\
\hline
\end{tabular}

DESIGNC.3

OESERVATIONS

$\begin{array}{lllllll}R V A T I O N S & 5 & 3 & 2 & 2 & 1 & 1 \\ Y(1) & + & - & + & - & - & - \\ Y(2) & + & - & - & + & - & - \\ Y(3) & + & - & - & - & + & + \\ Y(4) & + & - & - & & + & - \\ Y(5) & + & - & & - & + & - \\ Y(6) & + & - & - & & - & + \\ Y(7) & + & - & & - & - & + \\ Y(8) & + & - & & & - & - \\ Y(9) & + & & - & - & - & \\ Y(10) & + & & - & - & & - \\ Y(11) & & & + & - & & \end{array}$

RESTRAINT A

RESTRAINT 8

FACTCRS FCR COMPUTING ST DEVS

WT RESTRAINTS

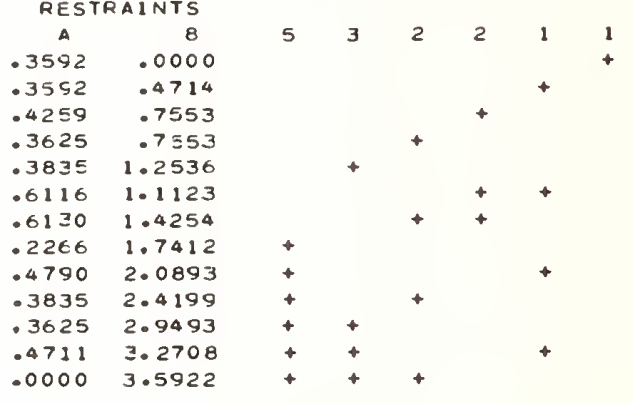

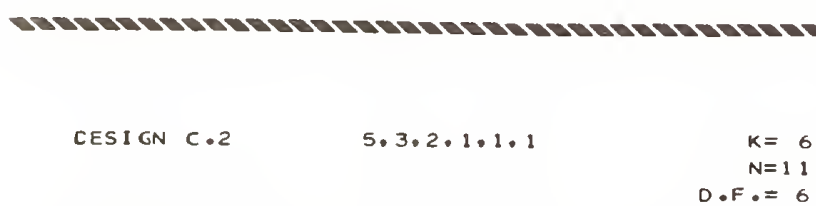

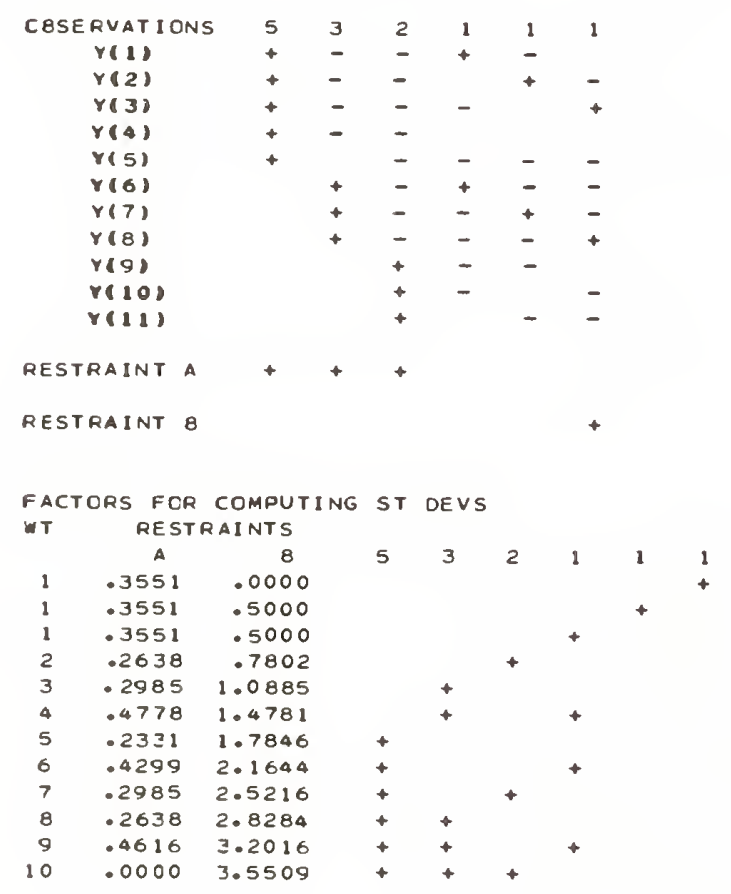

DESIGN C. 4

$5 \cdot 3 \cdot 2 \cdot 2 \cdot 1 \cdot 1$

$K=6$

$\mathrm{N}=12$

CBSERVATICNS

$r(1)$
$r(2)$
$r(3)$
$r(4)$
$r(5)$
$r(6)$
$r(7)$
$Y(8)$
$r(9)$
$r(10)$
$r(11)$
$r(12)$

RESTRAINT A

RESTRAINT 8

FACTORS FCR COMFUTING ST DEVS

WT RESTRAINTS

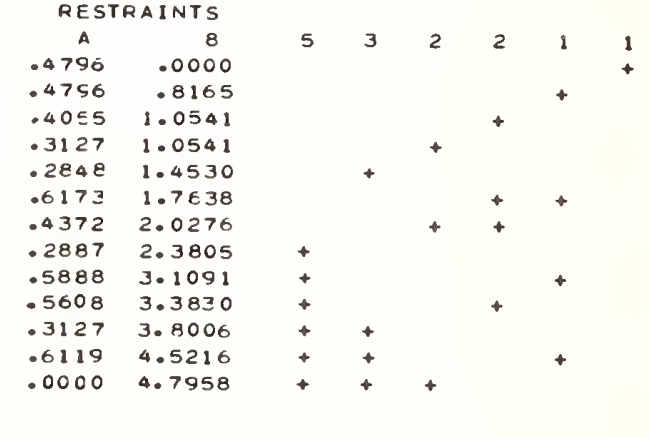

Figure 6. Least squares information for the design described in the text. (Reproduced from ref. [3]). 
1. The scatter in the data just taken is typical of the scatter found in previous measurements.

2. The mass of the standard weight used in the calibration design has not changed from its accepted value.

The $\mathrm{F}$ ratio and $\mathrm{t}$ value provide important tests of these two assumptions.

The $F$ ratio essentially monitors the precision of the measuring process. A detailed discussion is presented in Appendix D. We note that

$$
F=s^{2} / \sigma^{2}
$$

where $\sigma^{2}$ is the variance of the particular balance being utilized (based on a large collection of previous measurements). Generally, we expect $F$ to be close to 1 , but we must not be surprised if a particular value of $F$ is somewhat larger than 1 .

In particular, a simple check for $F$ can be (and has been) established for most mass metrology laboratories. By comparing $F$ with a fixed ratio $F_{t}$, which could be defined at the 99 percent confidence level one can then easily monitor whether the measured ratio $F$ is greater than $F_{t}$

$$
\text { i.e. } \quad F>F_{t} \text {. }
$$

If this check holds true, then the measurement process is considered "out of control" and further studies have to be conducted. The quantity $F_{t}$ depends only on the degrees of freedom $\left(k_{1}\right)$ in $s^{2}$ and on the probabiitity level at which we wish to conduct the test. The calculations are outlined in Appendix $D$.

The second test is called the " $t$ test" which monitors the accuracy of the measurements. For this test, an extra mass, the check standard, is included in the particular design. A simple check is then performed to see whether the mass value assigned to the check standard by the calibration agrees with the accepted value. The $t$ test thus monitors the systematic errors of the measuring process.

In particular one calculates

$$
t_{c}=\mid(\hat{S C})^{\top}-\text { accepted }(S C)^{\top} \mid / \sigma_{C}
$$

where

$(\hat{S C})^{\top}=$ observed mass of the check standard as found by least-squares fitting; accepted $(S C)^{\top}=$ accepted mass of the check standard;

and

$$
\sigma_{C}^{2}=l \sigma^{2}+\sigma_{T}^{2}+\left(N_{S C} / N_{R}\right)^{2} \sigma_{R}^{2} \text {. }
$$


Here

$\sigma_{T}^{2}=$ accepted variance of the measurement process between runs (see below)

$\ell=$ multiplier which is determined by the least squares process.

$\sigma=$ "process" standard deviation of the balance

$N_{S C}=$ the nominal value of the check standard

$N_{R}$ = the nominal value of the standard or transfer standard used as the restraint in the least squares solution

$\sigma_{R}=$ the standard deviation of the value of the restraint. $\sigma_{R}$ is taken as zero unless the restraint is a transfer standard, whose value was determined as part of the calibration of the complete weight set [1].

For simplicity, we will discuss a case for which $\sigma_{R}=0$. In general one checks to see that

$$
t_{c}<3
$$

The probability is less than one in 100 that this inequality will be violated by chance. Thus $t_{c}=3$ is taken as the control limit. Any measurement for which $t_{c}>3$ is considered "out of control" and is repeated.

CAUTION: The $t$ test is one of the best statistical measures of systematic errors available. Nevertheless, by their very nature, systematic errors are difficult to detect. If, for instance, buoyancy corrections were not important in assignment of mass to the check standard but were important in assigning mass values to the weights being calibrated, the $t$ test will not detect systematic errors in the buoyancy correction. Also, the $t$ test cannot detect an identical change in the standard and check standard. We have taken a very simple example of a $t$ test. The most general case is shown in ref. [1].

The variance of the measurement process between runs, $\sigma_{T}{ }^{2}$, is an important concept $[1,8]$. Recall that the process standard deviation, $\sigma$, is a measure of scatter over the period of time it takes to make a mass measurement: i.e., a few minutes to a few hours. There may, however, be sources of random uncertainty which fluctuate more slowly (i.e., over days or months); but still rapidiy compared with intervals between recalibration of a given weight. How can we estimate the process standard deviation for this longer period of time? The easiest way is to monitor the values of mass assigned to the check standard each time it is used in a run. After many runs, over a span of many months, we can estimate the variance, $\sigma_{c}{ }^{2}$, of the observed values of the check standard about their mean value.

If the process standard deviation has no "between-run" component, then

$$
\sigma_{c}^{2}=l \sigma^{2} \quad\left(\sigma_{T}^{2} \approx 0\right)
$$

where $\sigma^{2}$ is the process variance which was found by pooling variances estimated from the least squares fits to many individual runs. That is, $\sigma$ is the within-run process variance based on combining calculations of $s^{2}$ from many runs. 
It will generally be true, however, that

$$
\sigma_{c}^{2}>l \sigma^{2}
$$

indicating that there is an additional source of scatter from run to run. We define this between-run variance for the measurement process as

$$
\sigma_{T}^{2}=\sigma_{C}^{2}-\ell \sigma^{2} \text {. }
$$

If $\sigma_{T}^{2}$ is not negligible, the source of between-run scatter should, of course, be sought and eliminated if possible? Failing this, the process standard deviation must be expanded so that the over-all uncertainty assigned in the calibration report includes the between-run component.

The cautionary statement given above for the $t$ test also applies to the use of the check standard in estimating between-run scatter. That is, if a weight being calibrated differs markedly in its construction from the weight(s) used in the check standard, a $\sigma_{T}$ component may go undetected if one relies only on check-standard behavior.

Suppose a series of design data is "in control." True mass values have been assigned to the unknown weights and uncertainty limits have been given. What do these uncertainties mean? In a practical sense, the meaning is that the uncertainty bands assigned to each mass calibrated by the methods of ref. [1] should almost always overlap the uncertainty bands which would have been obtained had the masses been calibrated at NBS.

Note that this specification, while satisfying the expectation of the overwhelming majority of users, is somewhat less stringent than asserting that: the true mass as defined in eq. [1] and reported in S.I. units is almost surely within the uncertainty band provided by the calibration.

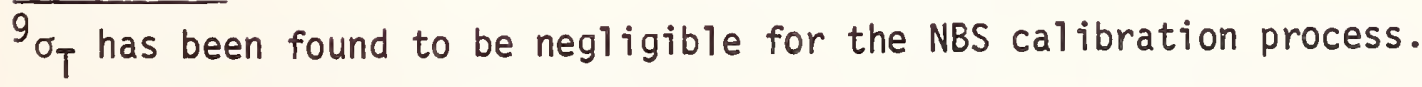




\section{CONCLUSION}

This document has been less of a complete treatise on mass metrology than a guide for the mass metrologist. We have indicated appropriate references where the various topics touched on are treated in greater detail.

What we have tried to offer is a coherent development of the fundamental concepts of mass metrology as a means of explaining the relevance to the metrologist of the references found at the end of this publication.

In brief, starting with the basic law of classical mechanics, we derived:

- Relations for the true mass differences between nominally equal weights (eqs. (10') and (19b))

- Relations for apparent mass differences between nominally equal weignis (eqs (18) and (19a))

We stressed the importance of measuring or calculating air density in order to make proper buoyancy corrections.

We then provided:

- An explanation of how the basic mass relations apply to measurements on several types of commonly used balances.

- A demonstration of how these relations are used in two types of surveillance testing.

- A brief sketch of how these relations are used in mass calibrations.

We concluded with a discussion of the statistical checks crucial to a calibration.

In a document such as this, there is a danger of losing the basic outlines of a mass measurement program in the many details essential to carrying out a successful program. Every program must have a goal--for example, the calibration of weights in a certain range of masses to a desired uncertainty.

One must then acquire balances equal to the task and house them in a room which will not degrade their performance. Control charts should be used to establish the longterm reliability of the balances. Calibrated sets of weights to be used as working standards should be acquired. Surveillance testing can establish the stability of these weights to within the surveillance limits.

Anticipated levels of buoyancy correction should be estimated. Auxillary equipment such as barometers, hygrometers, and thermometers should be acquired, if necessary, to achieve adequate capability in determining the density of air. These instruments must be calibrated periodically.

When calibrating weight sets, weighing designs should be used. These are selected on the basis of providing the necessary accuracy with the least number of weighing operations. Great care must be exercised in assigning a total uncertainty to calibration results.

The techniques outlined in this document form the core of such a mass program. 
We would like to express our appreciation to Dr. J.D. Simmons of NBS for his support. One of us (K.J.) wishes to thank R. Weber and Dr. D. Cauffman, both of Lockheed, for their encouragement, help, and overall support for this endeavor. Also, one of us (R.D.) extends thanks to his colleagues at NBS for their cooperation and technical guidance. We especially wish to thank Mrs. Betty King for typing the manuscript. 


\section{REFERENCES}

[1] Varner, R.N.; Raybold, R.C. National Bureau of Standards mass calibration computer software. Nat. Bur. Stand. (U.S.) Tech. Note 1127; 1980 July. 164 p. NTIS PB80203441

[2] Jones, Frank E. The air density equation and the transfer of the mass unit. $J$. Res. Nat. Bur. Stand. (U.S.) 83(5): 419-428; 1978 September-0ctober.

[3] Cameron, J. M.; Croarkin, M. C.; Raybold, R. C. Designs for the calibration of standards of mass. Nat. Bur. Stand. (U.S.) Tech. Note 952; 1977 June. 64 p. NTIS PB268499

[4] Almer, H. E., Keller, Jerry, ed. Surveillance test procedures. Nat. Bur. Stand. (U.S.) NBSIR 76-999; 1977 May. 77 p. NTIS PB268130

[5] Pontius, Paul E. Mass and mass values. Nat. Bur. Stand. (U.S.) Monogr. 133; 1974 January. 39 p. NTIS COM7450309

[6] Almer, H. E. Method of calibrating weights for piston gages. Nat. Bur. Stand. (U.S.) Tech. Note. 577; 1971 May. 54 p. NTIS COM7150264

[7] Precision measurement and calibration statistical concepts and procedures, Special Publication 300, Volume 1, Pontius, P.E., Cameron, J.M.; pp. 2-20. GP0 Stock No. 003-003-00072-8

[8] Pontius, P.E. Measurement philosophy of the pilot program for mass calibration. Nat. Bur. Stand. (U.S.) Tech. Note 288; 1966 May. 39 p. (Reprinted January, 1968).

[9] Schoonover, Randall M. A simple gravimetric method to determine barometer corrections. J. Res. Nat. Bur. Stand. (U.S.)85 (5) 341-345, 1980-SeptemberOctober.

[10] Schoonover, Randall M. A look at the electronic analytical balance. Anal. Chem. 52: 973A-980A; 1982 July.

[11] Schoonover, Randa11 M.; Jones, Frank E. Air buoyancy correction in high-accuracy weighing on analytical balances. Anal. Chem. 53: 900-902; 1981 May.

[12] Cameron, Joseph M.; Hailes, Geraldine E. Designs for the calibration of small groups of standards in the presence of drift. Nat. Bur. Stand. (U.S.) Tech. Note 844 ; 1974 August. 32 p. NTIS COM7450762

[13] Natrella, Mary Gibbons. Experimental statistics. Nat. Bur. Stand. (U.S.) Handb. 91; 1963 August (reprinted 1966 October with corrections). GPO Stock No. 0003-003-00135-0

[14] ASTM E617-81. Standard specifications for laboratory weights and precision mass standards. 1983 Annual Book of ASTM Standards Vol. 14.02: 455-474.

[15] Measurement Assurance Programs Part II: Development and Implementation, Nat. Bur. Stand. Special Publication 676II, Croarkin, C; 1984, 124 p. 
References with NTIS numbers are available from:

The National Technical Information Service Springfield, VA 22161

References with GPO stock numbers are available from:

The Superintendent of Documents U.S. Government Printing Office Washington, DC 20402 



\section{APPENDIX A \\ APPARENT MASS OF BUILT-IN BALANCE WEIGHTS}

Balance manufacturers almost always adjust the built-in dial weights of singlepan balances so that their apparent masses equal the value of the dial position. No matter of what density material the manufacturer has actually made his weights, the user generally assumes that the true masses equal the dial values and the densities of the built-in weights equal $\rho_{R}$, the basis density.

Let us see why this scheme works and what its limitations are. These considerations can be demonstrated by referring to a single dial-weight (of density $\rho_{D}$ ) that exactly balances a weight $X$, which is on the pan.

We begin, since we are discussing a balance of the type shown in fig. 3 with eq. (22):

$$
k \theta_{1} \doteq\left(M_{X}^{\top}-\rho_{A} V_{X}\right)-\left(\Sigma D_{i}^{\top}-\rho_{A} \Sigma V_{i}\right)+k \theta_{0},
$$

where we have renamed $M$ by $M_{X}$ and $V_{M}$ by $V_{X}$. We will assume that $\theta_{0}$ was adjusted to zero before $X$ was placed on the balance. We have chosen a special case: after $X$ was placed on the balance, the screen reading $\theta_{1}$ returned to zero with the removal of a single dial weight. Let us refer to this dial weight as "D" and say that it has a true mass $D^{\top}$ and a volume $V$ at the balance temperature. In this special case, with $\theta_{0}=\theta_{1}=0$, eq. (22) becomes

$$
0=\left(M_{X}^{\top}-\rho_{A} V_{X}\right)-\left(D^{\top}-\rho_{A} V\right)
$$

Since $\rho_{D}=D^{\top} / V$, we can rewrite $(A 1)$ as

$$
M_{X}^{\top}-\rho_{A} V_{X}=D^{\top}\left(1-\rho_{A} / \rho_{D}\right)
$$

or,

$$
M_{X}^{\top}=D^{\top}\left(1-\rho_{A} / \rho_{D}\right)+\rho_{A} V_{X} \equiv M_{1} .
$$

Now we ask the question, "What if one assumes the mass of $D$ is equal to $N_{D}$, its nominal value, and the density of $D$ is equal to $\rho_{R}$, the basis density of the apparent mass scale to which the balance weights have been adjusted?" This assumption is not correct because

$$
\rho_{R} \neq \rho_{D}
$$

and

$$
D^{\top} \neq N_{D}
$$


Nevertheless, the incorrect assumption would tell us that

$$
M_{X}^{\top}=N_{D}\left(1-\rho_{A} / \rho_{R}\right)+\rho_{A} V_{X} \equiv M_{2} \text {. }
$$

We can next ask ourselves "Under what conditions does $M_{1}=M_{2}$ ?"

Recalling that $M_{D}^{A}$ is made equal to $N_{D}$, the nominal dial value, we subtract eq.
(A3) from (A2).

$$
M_{1}-M_{2}=D^{\top}\left(1-\rho_{A} / \rho_{D}\right)-M_{D}^{A}\left(1-\rho_{A} / \rho_{R}\right) \text {. }
$$

Also, by definition, (see eq. ( 11) in the main text),

$$
M_{D}^{A}=D^{T}\left(1-\rho_{0} / \rho_{D O}\right) /\left(1-\rho_{0} / \rho_{R O}\right)
$$

where the subscript zeros refer to the standard conditions specified in the definition of apparent mass. Substituting (A5) into (A4),

$$
M_{2}-M_{1}=D^{\top}\left[\left(1-\rho_{0} / \rho_{D O}\right)\left(1-\rho_{A} / \rho_{R}\right) /\left(1-\rho_{0} / \rho_{R O}\right)-\left(1-\rho_{A} / \rho_{0}\right)\right] .
$$

The first approximation we will make is that $\rho_{R O}=\rho_{R}$ and $\rho_{D O}=\rho_{D}$. That is, the temperature is sufficiently close to $20{ }^{\circ} \mathrm{C}$ that the expansion in volume of the balance weights is negligible. Thus

$$
M_{2}-M_{1}=D^{\top}\left[\left(1-\rho_{0} / \rho_{D}\right)\left(1-\rho_{A} / \rho_{R}\right) /\left(1-\rho_{0} / \rho_{R}\right)-\left(1-\rho_{A} / \rho_{D}\right)\right] .
$$

Next, we use the relation developed in the main text:

$$
\left(1-\rho_{0} / \rho_{R}\right)^{-1}=1+\rho_{0} / \rho_{R}+\left(\rho_{0} / \rho_{R}\right)^{2}+\left(\rho_{0} / \rho_{R}\right)^{3}+\ldots
$$

so that

$$
M_{2}-M_{1}=D^{\top}\left[\left(1-\rho_{0} / \rho_{D}\right)\left(1-\rho_{A} / \rho_{R}\right)\left(1+\rho_{0} / \rho_{R}+\left(\rho_{0} / \rho_{R}\right)^{2}+\left(\rho_{0} / \rho_{R}\right)^{3}+\ldots\right)-\left(1-\rho_{A} / \rho_{D}\right)\right] .
$$

Finally, we multiply out but throw away all terms of order $\left(\rho_{0} / \rho_{R}\right)^{2},\left(\rho_{A} \rho_{0} / \rho_{R}^{2}\right)$, $\rho_{0}^{2} / \rho_{D} \rho_{R}$, and smaller. Since $\rho_{O} \sim \rho_{A} \leq 1.2 \mathrm{mg} / \mathrm{cm}^{3}$ and $\rho_{D} \sim \rho_{R}>7.5 \mathrm{~g} / \mathrm{cm}^{3}$ (the balance manufacturer has seen to this), then neglecting these terms leads to errors of $3 \times 10^{-6}$ percent or less.

Thus, we now have

$$
M_{2}-M_{1}=D^{\top}\left(\rho_{0} / \rho_{R}-\rho_{0} / \rho_{D}-\rho_{A} / \rho_{R}+\rho_{A} / \rho_{D}\right)
$$

or, more simply

$$
M_{2}-M_{1}=D^{\top}\left(\rho_{0}-\rho_{A}\right)\left(1 / \rho_{R}-1 / \rho_{D}\right)
$$


The balance manufacturer has selected the metal for his weights from stock having a density $\rho_{D}$ sufficiently close to $\rho_{R}$ so that, near sea level, $M_{1}-M_{2}$ will not
exceed the balance tolerance.

In recent years, balance manufacturers have been making weights of stainless steel. Equation (A6) shows why it is, therefore, desirable for these weights to be adjusted on the 8.0 basis.

The chief virtue of the apparent mass scheme is that, even though different balance manufacturers may make their weights of different alloys, a user can be completely unaware of these subtleties and still derive reasonably accurate true mass results on any balance so long as the correct basis density is used.

\section{Example:}

A balance has $20 \mathrm{~g}$ of built-in dial weights. The density of these weights is actually $7.8 \mathrm{~g} / \mathrm{cm}^{3}$. The weights have been adjusted so that the dial readings correspond to apparent mass on the brass basis. What is the value of $M_{2}-M_{1}$ at maximum load if $\rho_{A}=1.16 \mathrm{mg} / \mathrm{cm}^{3}$ ? What if the laboratory is at high elevation so that $\rho_{A}=1.00 \mathrm{~g} / \mathrm{cm}^{3}$ ?

\section{Answer:}
a) $M_{2}-M_{1}=20 \mathrm{~g}(0.00120-0.00116)\left[\frac{1}{8.4}-\frac{1}{7.8}\right]=-7 \mu \mathrm{g}$.
b) $M_{2}-M_{1}=20 \mathrm{~g}(0.00120-0.00100)\left[\frac{1}{8.4}-\frac{1}{7.8}\right]=-37 \mu \mathrm{g}$.

By comparison, the calibration uncertainty for high-quality $20 \mathrm{~g}$-weights as measured by NBS is abolit $10 \mu \mathrm{g}$; the tolerance for $20-\mathrm{g}$, Class 1 , metric weights is $74 \mu \mathrm{g}$ [14]. 



\section{OUTLINE OF THE DERIVATION OF $\rho_{A}$, THE DENSITY OF AIR}

In order to find $\rho_{A}$, an equation of state involving temperature, humidity, and barometric pressure has been developed. We will follow the definitive derivation of F. E. Jones [2] and briefly describe his arguments to arrive at a simplified relation sufficiently accurate for our applications. The reader is urged to consult [2] for full details.

In order to derive the density for a mixture of two gasses, let us start with the ideal gas law.

$$
\mathrm{pV}=\mathrm{nRT}
$$

and

$$
n=\frac{m}{M}
$$

where

$$
\begin{aligned}
& n=\text { number of moles } \\
& m=\text { mass of gas } \\
& M=\text { molecular weight of gas } \\
& R=\text { universal gas constant } \\
& T=\text { temperature in kelvins }=(273.15+t) \text { for } t \text { in degrees celsius. }
\end{aligned}
$$

From the above, we can rewrite

$$
\mathrm{pV}=\frac{\mathrm{m}}{\mathrm{M}} \mathrm{RT} \text { so that } \mathrm{m}=\frac{\mathrm{pVM}}{\mathrm{RT}}
$$

and

$$
\frac{M}{R T}=\frac{m}{p V}=\frac{\rho_{A}}{p} \text { since } \rho_{A}=\frac{m}{V} \text {. }
$$

Specifically, consider air consisting of dry air and water vapor. For the dry air, we have

$$
m_{D}=\frac{P_{D} V M_{D}}{R T}
$$

and for the water vapor, we have

$$
m_{w}=\frac{p_{w} V M_{w}}{R T} .
$$

Since the total density is given by

$$
\rho_{A}=\frac{m_{D}+m_{W}}{v}
$$


we obtain

$$
\begin{aligned}
\rho_{A} & =\left(\frac{p_{D} V M_{D}}{R T}+\frac{p_{W} V M_{W}}{R T}\right) / V \\
& =\frac{1}{R T}\left(p_{D} M_{D}+p_{W} M_{W}\right) .
\end{aligned}
$$

Using Dalton's Law for partial pressures

$$
P=p_{1}+p_{2}
$$

we can substitute for

$$
p_{D}=P-p_{W}
$$

so that

and using

$$
\begin{aligned}
\rho_{A} & =\frac{1}{R T}\left(P M_{D}-p_{W} M_{D}+p_{W} M_{W}\right) \\
& =\frac{M_{D}}{R T}\left(P+p_{W}\left(\frac{M_{W}}{M_{D}}-1\right)\right)
\end{aligned}
$$

$$
\begin{gathered}
\varepsilon=\frac{M_{W}}{M_{D}} \\
\rho_{A}=\frac{M_{D}}{R T}\left(P+p_{W}(\varepsilon-1)\right) .
\end{gathered}
$$

At this point it is important to consider for a moment the correction necessary for a gas which is not ideal. To do so we rewrite the last relation as

$$
P=\frac{\rho_{A} R T}{M_{D}} \frac{1}{\left[1+\frac{P_{W}}{P}(\varepsilon-1)\right]}
$$

For an ideal gas the ratio

$\rho_{A} \frac{R T}{M_{D}\left[\frac{P}{1+\frac{p_{W}}{P}(\varepsilon-1)}\right]}=Z$


has to be equal to 1 . If we want to consider non-ideal gas corrections, then we have to incorporate $Z$ into our formalism. Hence,

$$
P=\frac{\rho_{A} R T Z}{M_{D}} \frac{1}{\left[1+\frac{P_{W}}{P}(\varepsilon-1)\right]}
$$

where $Z$ is called the "compressibility factor."

Rewriting this relation for the mixture density, we now have

$$
\rho_{A}=\frac{M_{D}}{R T Z}\left[\left(P+(\varepsilon-1) p_{W}\right] .\right.
$$

Let us now turn to the dependence of this relation on the relative humidity. We define

$$
U=\frac{P_{W}}{e_{S}^{1}} \times 100
$$

where

$$
\begin{aligned}
& U=\text { relative humidity in percent } \\
& \mathrm{P}_{W}=\text { effective vapor pressure of water in moist air } \\
& \mathrm{e}_{S}^{1}=\text { effective saturation vapor pressure of water in moist air. }
\end{aligned}
$$

Furthermore, we know that

$$
e_{s}^{\prime}>e_{s}
$$

where

$$
\mathrm{e}_{\mathrm{s}}=\text { the saturation vapor pressure of pure-phase water. }
$$

The ratio of the two pressures is called the "enhancement factor" for saturated water vapor and is given by

$$
f=\frac{e_{s}^{1}}{e_{s}}
$$

so that

$$
e_{s}^{\prime}=e_{s} \cdot f
$$

and since

$$
p_{W}=\frac{U \cdot e_{s}^{\prime}}{100}
$$


we have

$$
p_{W}=\frac{\dot{U} \cdot e_{S} \cdot f}{100}
$$

Substitution into the mixture density relation yields

$$
\rho_{A}=\frac{M_{D}}{R T Z}\left[P+(\varepsilon-1) \frac{U \cdot e_{s} \cdot f}{100}\right]
$$

where $U$ must be given in $\%$.

This relation constitutes the final formula. The parameters $P, T$, and $U$ must be measured by the user.

Substituting the best available values for $R$ anf $M_{W}$; and choosing reasonable values for $M_{D}, Z$, and $f$, which are approximated as constant parameters, we have

$$
\begin{aligned}
& R=8.31441 \frac{\text { joules }}{\text { mole-K }}=8,374.4 \frac{\text { joules }}{\text { kmole-K }} \\
& M_{D}=28.964 \mathrm{~g} / \mathrm{mole} \\
& Z=0.9996 \\
& f=1.0042 \\
& M_{W}=18.0152 \mathrm{~g} / \mathrm{mole} .
\end{aligned}
$$

Since

$$
\varepsilon=\frac{M_{W}}{M_{D}}=\frac{18.0152}{28.964}
$$

we obtain

$$
\begin{aligned}
\rho_{A} & =\frac{3.4848}{T}\left(P-0.0037960 U_{\mathrm{e}}\right) \times 10^{-3} \\
\rho_{A}\left(\frac{\mathrm{mg}}{\mathrm{cm}^{3}}\right) & =\frac{0.0034848}{(t+273.15)}\left(P-0.0037960 U_{e_{s}}\right) .
\end{aligned}
$$

where

$$
\begin{aligned}
& t \text { is in }{ }^{\circ} \mathrm{C} \\
& \mathrm{P} \text { is in pascals }(133.3224 \mathrm{~Pa}=1 \mathrm{~mm} \mathrm{Hg}) .
\end{aligned}
$$

Converting to $\mathrm{mm} \mathrm{Hg}$ pressure, we have

$$
\rho_{A}\left(\frac{\mathrm{mg}}{\mathrm{cm}^{3}}\right)=\frac{0.46460}{(t+273.15)}\left(P-0.0037960 \mathrm{Ue}_{\mathrm{s}}\right) \text {. }
$$


The parameter $e_{\mathrm{s}}$ has been determined by fitting measured data between $288.15 \mathrm{~K}$ and $301.15 \mathrm{~K}$. The relationship developed is

$$
e_{S}=\left(1.3146 \times 10^{9}\right) e^{\frac{-5315.56}{(t+273.15)}} \mathrm{mm} \mathrm{Hg} \text {. }
$$

It should also be noted that the value for the enhancement factor used above,i.e. $f=1.0042$ can be approximated more accurately by

$$
f=1.00070+4.150 \times 10^{-6} \times P+5.4 \times 10^{-7} t^{2} .
$$

In the above final equation, the relative humidity is given in percent

$$
U=\% \text { (e.g. } 51.2,43.7 \text { etc.) }
$$

and the pressure $P$ in $\mathrm{mm} \mathrm{Hg}$.

$M_{D}$ (and, therefore, $\varepsilon$ ) depends on the mixture of gases, other than water vapor, which makes up ambient air. The chief variability in this mixture comes from $\mathrm{CO}_{2}$ and $\mathrm{O}_{2}$ levels. These are assumed perfectly correlated--that is, $\mathrm{CO}_{2}$ levels can only increase locally at the expense of $\mathrm{O}_{2}$ and vice versa--as in processes of combustion, respiration and photosynthesis.

Fortunately, the variation has little effect on $M_{D}$. In eqs. (20a) and (20b) (main text) we have assumed the ambient level of $\mathrm{CO}_{2}$ typically found in the NBS mass laboratories $\left(0.00042 \mathrm{~mol}\right.$ of $\mathrm{CO}_{2} / \mathrm{mol}$ of air). A 100 percent increase in this level would raise $\rho_{A}$ by less than 0.02 percent.

$Z$ is slightly dependent on barometric pressure, temperature and relative humidity [2]. Between $19{ }^{\circ} \mathrm{C}$ and $26^{\circ} \mathrm{C} ; 525 \mathrm{~mm} \mathrm{Hg}$ and $825 \mathrm{~mm} \mathrm{Hg}$; and $\mathrm{O}$ and $100 \%$ R.H., $Z$ varies by less than 0.03 percent. 

Using eq. (20b) (developed in Appendix B), we have

$$
\rho_{A}=\frac{0.46460}{(t+273.15)}\left(P-0.0037960 \cdot U \cdot e_{s}\right) \text {. }
$$

For ease of writing, we will set

$$
\begin{aligned}
& \rho=\rho_{A} \\
& a=0.46460 \\
& b=273.15 \\
& c=0.0037960 \\
& e=e_{S}
\end{aligned}
$$

such that

$$
\rho=\frac{a}{(t+b)}(p-c \cdot U \cdot e) .
$$

We want to determine the uncertainty for $\rho$ using

$$
d \rho=\frac{\partial \rho}{\partial t} d t+\frac{\partial \rho}{\partial P} d P+\frac{\partial \rho}{\partial U} d U \text {. }
$$

$\underline{\partial \rho}:$

$\underline{\partial t}$

Using $(\mathrm{C} 1)$, we find

$$
\begin{aligned}
\frac{\partial \rho}{\partial t} & =a(P-c \cdot U \cdot e) \frac{\partial}{\partial t}\left(\frac{1}{t+b}\right) \\
& =\left(\frac{-1}{t+b}\right) \rho
\end{aligned}
$$

$\underline{\partial \rho}:$

$\partial P$

Using $(\mathrm{Cl})$, we find

$$
\frac{\partial \rho}{\partial P}=\frac{a}{t+b}
$$

and since

$$
\rho \approx \frac{a p}{(t+b)}
$$


where we ignored the second term in (Cl) since it is only a secondary source of error, we have

$$
\frac{\partial \rho}{\partial P} \approx \frac{\rho}{P}
$$

$\underline{\partial \rho}$ :

$\underline{\partial U}$

From $(\mathrm{C} 1)$, we obtain

$$
\rho=\frac{a P}{t+b}-\frac{a c e U}{t+b}
$$

so that

$$
\frac{\partial \rho}{\partial U}=-\frac{a c e}{(t+b)}
$$

and again using $\rho \frac{a P}{t+b}$

we have

$$
\frac{\partial \rho}{\partial U} \approx \frac{-c e \rho}{P} .
$$

Next, we have to estimate the combined uncertainties due to $t, P$, and $U$. In Section 2.3.2 (main text) we asserted that the temperature should be known to $\pm 0.4{ }^{\circ} \mathrm{C}$ so that

$$
d t= \pm 0.4^{\circ} \mathrm{C} \text {. }
$$

Similariy,

$$
\mathrm{dP}= \pm 7.1 \mathrm{~mm} \mathrm{Hg}
$$

and

$$
d U= \pm 16 \% .
$$

A11 these values assume that we want to know the air density to $0.0017 \mathrm{mg} / \mathrm{cm}^{3}$. Substitution of all terms into (C2) yields

$$
d \rho=\frac{-\rho}{t+b}( \pm 0.4)+\frac{\rho}{p}( \pm 1.1)-\frac{c e}{p} \rho( \pm 16) .
$$

In the worst case, these uncertainties would add linearly. However, we assume the errors are uncorrelated so that a better estimate of the total uncertainty is given by

$$
\delta \rho=\sqrt{\left(\frac{\partial \rho}{\partial t} d t\right)^{2}+\left(\frac{\partial \rho}{\partial P} d P\right)^{2}+\left(\frac{\partial \rho}{\partial U} d U\right)^{2}}
$$




$$
\begin{aligned}
& =\sqrt{\frac{\rho^{2} \cdot(0.4)^{2}}{(t+b)^{2}}+\frac{\rho^{2}(1.1)^{2}}{p^{2}}+\frac{c^{2} e^{2} \rho^{2}(16)^{2}}{p^{2}}} \\
& =\rho \sqrt{\frac{0.16}{(t+b)^{2}}+\frac{1.21}{p^{2}}+\frac{c^{2} e^{2} 256}{p^{2}}} .
\end{aligned}
$$

At standard conditions we have

$$
\begin{aligned}
& t=20^{\circ} \mathrm{C} \\
& P=760 \mathrm{~mm} \mathrm{Hg} .
\end{aligned}
$$

We now have

$$
\delta \rho=\rho \sqrt{\frac{0.16}{(20+b)^{2}}+\frac{1.21}{(-760)^{2}}+\frac{c^{2} e^{2} 256}{(760)^{2}}} .
$$

Substituting $\rho=\rho_{A}$ and replacing $b$ and $c$ with their numerical values, we have

$$
\frac{\delta \rho_{A}}{\rho_{A}}=\sqrt{\frac{0.16}{(20+273.15)^{2}}+\frac{1.21}{(760)^{2}}+\frac{(0.0037960)^{2} \cdot 256 \cdot e_{S}^{2}}{(760)^{2}}} .
$$

Also $e_{s}$ at $20^{\circ} \mathrm{C}$ is $17.54 \mathrm{~mm} \mathrm{Hg}$, so that finally

$$
\frac{\delta \rho_{A}}{\rho_{A}} \simeq 0.0024
$$

or

$$
\frac{\delta \rho_{A}}{\rho_{A}} \simeq 0.24 \%
$$





\section{SOME STATISTICAL CONCEPTS}

We start out with an infinitely large population of a random variable; to be practical, let us say a very large number of similar balance observations. We assume that this population is normally distributed with a mean of $\mu$ and a variance of $\sigma^{2}$.

Let us take a random sample of size $n$ from the population. Then the mean of the sample is defined as

$$
x=\frac{1}{n} \sum_{i=1}^{n} x_{i}
$$

with an estimated variance for a single observation of

$$
s^{2}=\frac{\sum_{i=1}^{n}\left(x_{i}-\bar{x}\right)^{2}}{n-1}
$$

As $n$ grows infinitely large $\bar{x} \rightarrow \mu$ and $s^{2} \rightarrow \sigma^{2}$. (This, in fact, defines what we mean by $\mu$ and $\sigma$ ).

The treatment of least squares data is more complicated than analysis of repeated measurements of the same quantity. In this case, average values of several different masses are calculated at the same time from data obtained during one weighing design. If $\mathrm{N}$ measurements were necessary to complete the design, the metrologist has in hand the results of these $\mathrm{N}$ mass comparisons: $\delta_{1}, \delta_{2}, \ldots \delta_{\mathrm{N}}$. The least squares analysis provides a set of "best" estimates: $\delta_{j}^{\prime}, \delta_{2}^{1}, \ldots \delta_{N}^{\prime}$, as well as a set of "best" estimates: $M_{7}^{\top}, M_{2}^{\top}, \ldots M_{K}^{\top}$, of the $K$ unknown masses calibrated by means of the design. These "best" estimates are linear combinations of the measured values $\delta_{1}, \delta_{2}, \ldots \delta_{N}$ with the mass of the known standard used as the restraint. The linear combinations are uniquely determined by the choice of design and restraint. Least squares solutions to the most useful weighing designs are tabulated in ref. [3].

The standard deviation of the least squares fit to the design data is estimated from the formula ${ }^{1}$

$$
s^{2}=\frac{\sum_{i=1}^{N}\left(\delta_{i}-\delta_{i}^{\prime}\right)^{2}}{N-K+1}
$$

where the denominator is given by the degrees of freedom (= number of observations number of unknowns + number of restraints). If the same measurement design were repeated m times, a better estimate for the standard deviation of the process could be obtained:

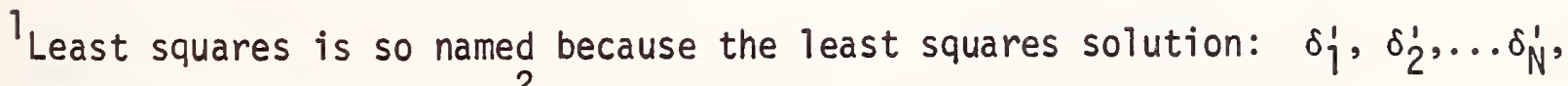
minimizes the value of $s^{2}$ in (D3). 


$$
s^{2}=\frac{s_{1}^{2}+s_{2}^{2}+\ldots+s_{m}^{2}}{m}
$$

where $s_{j}^{2}$ is the estimated variance of the $i^{\text {th }}$ run. As $m \rightarrow \infty, s^{2} \rightarrow \sigma^{2}$. This defines what we mean by $\sigma^{2}$ in the case of least squares. Note: the number of degrees of freedom (D.F.) in $s^{2}$ as defined by $(D 4)$ is $m(N-K+1)$. Also, this $\sigma$ is the same $\sigma$ discussed in the text, the "process standard deviation."

Besides $\sigma$, we would also like to know the standard deviation of each individual mass, $M_{j}$, computed from the least squares analysis. The answer involves matrix algebra and depends both on the design and restraint which were used, as well as on $\sigma$. All of the commonly used cases are tabulated in ref. [3]. In general, the standard deviation of a measured mass $\hat{M}_{j}$ is some value $\ell_{j} \sigma$, where $l_{j}$ is a number that depends only on the particular design and restraint. This number can be found in ref. [3]; $\sigma$ is defined above.

When two or more weights--j and $p$ for example--are used in combination, their combined standard deviation is not simply $\left(l_{j}^{2}+l_{p}^{2}\right)^{1 / 2} \sigma$ but is often somewhat greater. This is because data taken from a design are usually correlated. Both the recipe for handling weight summations and tabulated values for many special cases can be found in ref. [3].

From the above discussion, it should be evident that care must be taken in choosing the design and restraints for any set of weights to be calibrated. Some choices will minimize individual $\ell_{i}$ values but weight summations may have large uncertainties.

Assume that an estimate of $\sigma^{2}$ has been computed from $m$ designs by application of eq. (D4). Future designs will produce individual values $s_{j}^{2}$ which will also be estimates of $\sigma^{2}$ as long as the measurement process remains stable. An $F$ statistic

$$
F=\frac{s_{j}^{2}}{s^{2}}
$$

can be computed for each new $s_{j}^{2}$. Its purpose is to test the agreement between $s_{j}^{2}$ and $s^{2}$--or more precisely to test whether or not $s_{j}^{2}$ comes from the same distribution of measurements that produced $s^{2}$.

Given a large number of $F$ statistics, each of which is based on the same design, a histogram of these $F$ statistics will fall very nearly on the universal curve known as the $F$ distribution. The theoretical calculation of this curve depends only on the degrees of freedom in each $s_{j}^{2}$, i.e. $N-K-1$, and on the degrees of freedom in $s^{2}$, i.e. $m(N-K-1)$. The distribution is scaled so that the area under the curve is equal to 1, making it possible, for example, to find the point $F_{t}$ such that 99 percent of all $F$ values will be less than $F_{t}$. As a consequence, the next time that we carry out the weighing design, we expect that there is a 99 percent chance that the $F$ statistic computed from that particular design will be less than the percent point $F_{t}$. If, in fact, it turns out that the $F$ statistic is greater than the percent point $F_{t}$, the 
precision for that design is poorer than is expected, and the design should be repeated.

The percent point $F_{t}$ is often represented in tables as $F\left(\nu_{1}, v_{2}, 1-\alpha\right)$ because it depends on:

$$
\begin{aligned}
& v_{1}=\text { the degrees of freedom in } s_{j}^{2} \text { which is } N-K+1 \\
& v_{2}=\text { the degrees of freedom in } s^{2} \text { which is } m(N-K+1) \\
& \alpha=\text { the significance level such as } \alpha=0.01 \text { (i.e. } 99 \text { percent) }
\end{aligned}
$$

For mass calibrations at NBS, the significance level is chosen to be $\alpha=0.01$, and because the measurement process has been tracked for a long time resulting in an estimate of $\sigma^{2}$ that has a very large number of degrees of freedom, the value that is used for the test is

$$
F_{t}=F\left(\nu_{1}, \infty, 0.99\right)
$$

In this special case, $F_{t}$ can be well-approximated by*:

$$
F_{t}=\left(1-\frac{2}{9(N-K+1)}+2.32635 \sqrt{\frac{2}{9(N-K+1)}}\right)^{3}
$$

for

$$
v_{1} \geq 2
$$

and

$$
F_{t}=6.64
$$

for

$$
v_{1}=1 \text {. }
$$

Tables and detailed discussion on the $F$ value and $t$ ratio can be found in ref. [13].

\footnotetext{
* A derivation of this result is well beyond the scope of this work. The interested reader may wish to consult: Paulson, Edward. An approximate normalization of the analysis of variance distribution. Annals of Mathematical Statistics. 13: 223-235; 1942.
} 


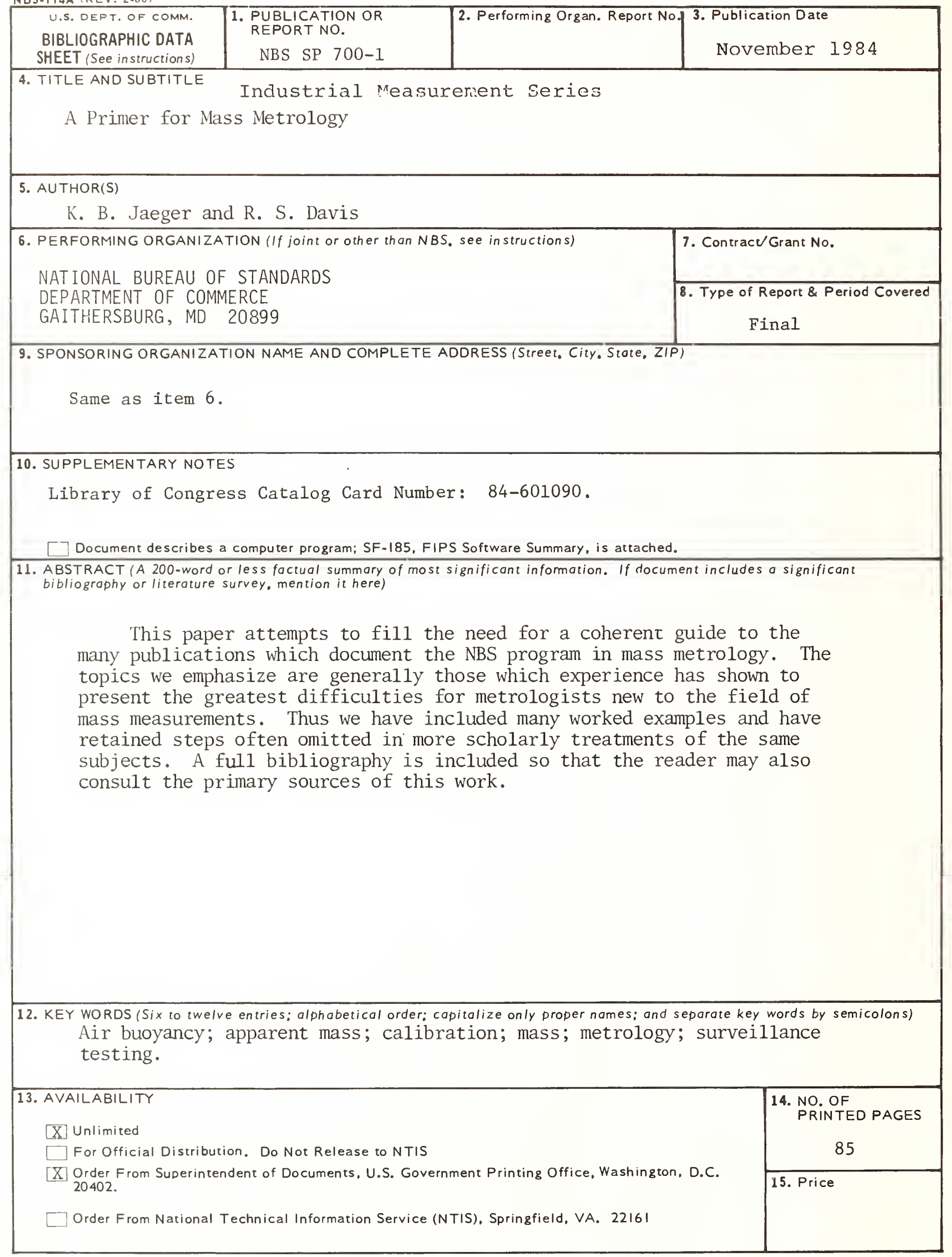




\section{Technical Publications}

\section{Periodicals}

Journal of Research-The Journal of Research of the National Bureau of Standards reports NBS research and development in those disciplines of the physical and engineering sciences in which the Bureau is active. These include physics, chemistry, engineering, mathematics, and conputer sciences. Papers cover a broad range of subjects, with major emphasis on measurement methodology and the basic technology underlying standardization. Also included from time to time are survey articles on topics closely related to the Bureau's technical and scientific programs. As a special service to subscribers each issue contains complete citations to all recent Bureau publications in both NBS and non-NBS media. Issued six times a year.

\section{Nonperiodicals}

Monographs-Major contributions to the technical literature on various subjects related to the Bureau's scientific and technical activities.

Handbooks-Recommended codes of engineering and industrial practice (including safety codes) developed in cooperation with interested industries, professional organizations, and regulatory bodies.

Special Publications-Include proceedings of conferences sponsored by NBS, NBS annual reports, and other special publications appropriate to this grouping such as wall charts, pocket cards, and bibliographies.

Applied Mathematics Series-Mathematical tables, manuals, and studies of special interest to physicists, engineers, chemists, biologists, mathematicians, computer programmers, and others engaged in scientific and technical work.

National Standard Reference Data Series-Provides quantitative data on the physical and chemical properties of materials, compiled from the world's literature and critically evaluated. Developed under a worldwide program coordinated by NBS under the authority of the National Standard Data Act (Public Law 90-396).

NOTE: The Journal of Physical and Chemical Reference Data (JPCRD) is published quarterly for NBS by the American Chemical Society (ACS) and the American Institute of Physics (AIP). Subscriptions, reprints, and supplements are available from ACS, 1155 Sixteenth St., NW, Washington, DC 20056.

Building Science Series-Disseminates technical information developed at the Bureau on building materials, components, systems, and whole structures. The series presents research results, test methods, and performance criteria related to the structural and environmental functions and the durability and safety characteristics of building elements and systems.

Technical Notes-Studies or reports which are complete in themselves but restrictive in their treatment of a subject. Analogous to monographs but not so comprehensive in scope or definitive in treatment of the subject area. Often serve as a vehicle for final reports of work performed at NBS under the sponsorship of other government agencies.

Voluntary Product Standards-Developed under procedures published by the Department of Commerce in Part 10, Title 15, of the Code of Federal Regulations. The standards establish nationally recognized requirements for products, and provide all concerned interests with a basis for common understanding of the characteristics of the products. NBS administers this program as a supplement to the activities of the private sector standardizing organizations.

Consumer Information Series-Practical information, based on NBS research and experience, covering areas of interest to the consumer. Easily understandable language and illustrations provide useful background knowledge for shopping in today's technological marketplace.

Order the above NBS publications from: Superintendent of Documents, Government Printing Office, Washington, DC 20402.

Order the following NBS publications-FIPS and NBSIR's-from the National Technical Information Service, Springfield, VA 22161.

Federal Information Processing Standards Publications (FIPS PUB)-Publications in this series collectively constitute the Federal Information Processing Standards Register. The Register serves as the official source of information in the Federal Government regarding standards issued by NBS pursuant to the Federal Property and Administrative Services Act of 1949 as amended, Public Law 89-306 (79 Stat. 1127), and as implemented by Executive Order 11717 (38 FR 12315, dated May 11, 1973) and Part 6 of Title 15 CFR (Code of Federal Regulations).

NBS Interagency Reports (NBSIR)-A special series of interim or final reports on work performed by NBS for outside sponsors (both government and non-government). In general, initial distribution is handled by the sponsor; public distribution is by the National Technical Information Service, Springfield, VA 22161, in paper copy or microfiche form. 
U.S. Department of Commerce

National Bureau of Standards

Gaithersburg, MD 20899

Official Business

Penalty for Private Use $\$ 300$ 